\title{
Polarized Compton Scattering off the Nucleon
}

\author{
Dissertation \\ zur Erlangung des Doktorgrades \\ der Mathematisch-Naturwissenschaftlichen Fakultäten \\ der Georg-August-Universität zu Göttingen
}

\author{
vorgelegt von \\ Nan Young Lee \\ aus Seoul, Korea
}

Göttingen 2001 
D7

Referent: $\quad$ Prof. Dr. M. Schumacher

Koreferent: Prof. Dr. F. Smend

Tag der mündlichen Prüfung: 27. 06. 2001 


\section{Contents}

1. Introduction

2. Compton scattering amplitude and fixed- $t$ unsubtracted DR $\quad 7$

2.1. Defintion and properties of helicity . . . . . . . . . . . . . . 7

2.2. Kinematics . . . . . . . . . . . . . . . . . . . . 8

2.3. Helicity amplitudes . . . . . . . . . . . . . . . . . . . . . . . . . . . . . . . .

2.3.1. Partial wave expansion . . . . . . . . . . . . . . . . . . . . 11

2.4. Parity conservation and multipoles . . . . . . . . . . . . . 12

2.4.1. Unitarity bound . . . . . . . . . . . . . . . . . . . . . . . . . . . . . . . . . . .

2.5. Invariant amplitudes . . . . . . . . . . . . . . . . . . . 16

2.6. Fixed-t dispersion relation . . . . . . . . . . . . . . . . . . 17

2.6.1. Non-Born contribution . . . . . . . . . . . . . . . 18

3. Low Energy Theory 23

3.1. Spin polarizabilities . . . . . . . . . . . . . . . . . . . 23

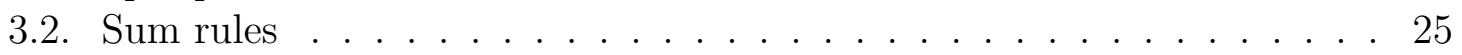

3.3. Comparision with theoretical predictions . . . . . . . . . . 26

4. Polarized nucleon Compton scattering 31

4.1. Polarization matrix . . . . . . . . . . . . . . . . . . . . 32

4.1 Photon . . . . . . . . . . . . . . . . . . 32

4.12 Nucleon . . . . . . . . . . . . . . . . . . . . 33

4.2. Polarization observables . . . . . . . . . . . . . . . . . . 34

4.2 .1 Generalforms . . . . . . . . . . . . . . . . . . 34

4.2.2. Formalismus in helicity and invariant amplitudes . . . . . . . . 37

$4.2 .3 . \quad$ Observables by multipole representation . . . . . . . . . . . . 48

4.3. LET of the observables in the c.m. system . . . . . . . . . . . . . . 50

4.4. Numerical results and Discussion . . . . . . . . . . . . . . . . 54

$\begin{array}{ll}\text { 5. Summery } & 67\end{array}$

\begin{tabular}{ll}
\hline A. Mandelstam variables & 68
\end{tabular} 
B. Symmetry property of $d$-function 70

$\begin{array}{ll}\text { C. Unsubtracted dispersion relation } & \mathbf{7 1}\end{array}$

C.1. Basic assumptions . . . . . . . . . . . . . . . . . . . . . 71

C2. Derivation of hasic DR . . . . . . . . . . . . . . 71

D. Relations between invariant, helicity and multipole amplitudes 73

E. Compton scattering amplitudes $R_{i}$ in center of mass system 76

F. Pauli and Dirac matrices $\quad 78$

E.l. Paulimatrix . . . . . . . . . . . . . . . . . . . . . 78

F.1.1. spin matrix . . . . . . . . . . . . . . . . . . . . . . . . . 78

E2. Dirac martrix . . . . . . . . . . . . . . . . . . . 79 


\section{Introduction}

The smallest entities of strongly interacting matter are hadrons, either baryons that are aggregated of three quarks or mesons that are made from quark-antiquark pairs. The most stable baryons are protons and neutrons that are the major constituents of atomic nuclei together with electrons. And the lightest meson is the pion.

A major goal of hadron physics is an understanding of the structures of hadronic matter and their dynamical properties whose underlying theory is quantum chromodynamics (QCD). This interest follows two paths. One is concerned with the properties of families of hadrons as they exist freely, to accurately characterize the members of the hadron spectrum in mass and decay properties and to reflect the structures that arise from QCD. The other is to understand how these properties change when hadrons are immersed in a nuclei or a nuclear matter.

The coherent-elastic photon scattering, called Compton scattering, is a clean tool to investigate the internal structure of the nucleons, the nuclei and the nucleon-nucleon interaction for the following reasons: i) The photon has only the electromagnetic interaction with other objects that can be comprehended physically quite well. ii) Furthermore, the transversity of a real photon simplifies the interpreting of the experimental data. iii) On the experimental side, there has also been made considerable progress with the help of developments of the direct current electron accerlator and the energy tagging spectrometer.

The scattering process can be described by the S-matrix (scattering matrix) which transformes an initial state into a final state, where the particles may be treated as noninteracting ones in these states. The properties of the S-matrix elements may be derivated from some general physical assumptions such as the unitarity and the causality. The unitarity condition says that the sum of the probabilities for all possible intermediate states must be equal to unity. The causality can be formulated such that the effect cannot proceede the cause. In other words, some quantity vanishes over a range of values of its argument. This fundamental hypothesis leads to analytic property of the S-matrix elements when the variables on which they expand, i.e. energies and momenta of the particles, are extended to the complex values. And the application of Cauchy's integral to such analytic function results in a dispersion relation (DR). 
The dispersion relations, which are a particularly suitable mathematical technique for dealing with the strongly interacting particles, provide a convienient language for a semiphenomenological description of an experiment. For example, the forward Compton scattering amplitude is expressed via the total photoabsorption cross section by virtue of the optical theorem and a dispersion relation.

In order to get more information on the angle distribution of the scattering amplitude $f$, we can decompose the amplitude $f$ into the various partial waves which can be transformed with each other. We will investigate the structure and the properties of the three decomposed amplitudes, called in this work the helicity, the multipole and the invariant amplitudes respectively, in the next chapter. In the third chapter, we will calculate the four parameters $\gamma_{M 1}, \gamma_{E 1}, \gamma_{M 2}$ and $\gamma_{E 2}$ called spin polarizabilities, based on the fixed- $t$ unsubtracted dispersion relation, which characterize the photon scattering amplitude by the nucleon in the limit of the photon energy $\omega \rightarrow 0$ together with the electric $\alpha$ and magnetic $\beta$ polarizability. The numerical results will be compared with the theoretical prediction of Heavy Baryon Chiral Perturbation Theory (HBChPT).

If the photon or the target or the both of them are polarized in an intial or in a final state, one can measure the asymmetries in addition to an unpolarized cross section. These polarized observables, that is these additionally measurable quantities, serve to provide information on the polarizabilities as well as the admixture ratio of the electric quadrupole and magnetic dipole $E 2 / M 1$ in $\Delta$-region. With the help of the photon and the nucleon polarization matrix, we will compute the observables of the polarized Compton scattering, and demonstrate numerical results for them together with the experimental data in the fourth chapter. In particular, we will present the kinematic regions, where the observables are especially sensitive to the parameters of the polarizabilities and the $E 2 / M 1$ mixing ratio.

The results of the investigations are summerized again, in the last chapter.

In this work, we use such units that $\hbar=c=m=1$. 


\section{Compton scattering amplitude and fixed- $t$ unsubtracted DR}

In a non-relativistic quantum mechanics, an intrinsic angular momentum of a particle, or spin $\boldsymbol{s}$, is introduced as an additional rotational degree of freedom decoupled from the usual kinematic degree of freedom like an orbital angular momentum $\boldsymbol{l}$. In a momentum representation, the state vector of a particle with a momentum $\boldsymbol{p}$ and a spin $\boldsymbol{s}$ is thus written in the form $|\boldsymbol{p} ; \boldsymbol{s} m>=| \boldsymbol{p}>\otimes \mid \boldsymbol{s} m>$, where $\mid \boldsymbol{s} m>$ are the simultaneous eigenstates of the operators $\hat{\boldsymbol{s}}^{2}$ and $\hat{s}_{z}$ with eigenvalues $s(s+1)$ and $m$, respectively.

In a relativistic process, a spin state of a particle having a non-vanishing rest mass may be labeled exactly as in the non-relativistic case by using the spin operators as defined in Refs. [[, [13]. While for massless particles, e.g. photon, which move with the velocity of light, the definition of the spin as an angular momentum of a particle at rest frame cannot be applied any longer, because there is no fixed reference system for these particles. In a relativistic quantum theory it is therefore not trivial to see how spin is to be described, nor how it is to be interpreted physically. To avoid these complications, a spin state is in this work classified by means of the quantum number $\lambda$ called helicity. This method is available to particles with zero mass as well as to massive particles.

\subsection{Defintion and properties of helicity}

A helicity is defined as the spin component along a direction of momentum $\hat{\boldsymbol{n}}=\boldsymbol{p} /|\boldsymbol{p}|$ of a particle:

$$
\lambda=\boldsymbol{s} \cdot \hat{\boldsymbol{n}} .
$$

In a relativistic theory, an orbital angular momentum $\boldsymbol{l}$ and a spin $\boldsymbol{s}$ for a free particle are not seperately conserved, but only the total angular momentum $\boldsymbol{j}$ is conserved [84, 50]. Therefore, $\lambda$ can also be defined as a component of the total angular momentum in the direction of a motion:

$$
\lambda=\boldsymbol{j} \cdot \hat{\boldsymbol{n}}
$$

The helicity states have the following properties under the transformations of a rotation, a reflection and the proper Lorentz transformation [7, 54]: 
1. For a massive particle there are $(2 s+1)$ independent helicity states, i.e. $(2 s+1)$ values of $\lambda$ for a given $\boldsymbol{p}$, while for a massless particle only two independent helicity states $\lambda= \pm s$ are allowed.

2. Under the ordinary rotation of the $x y z$-axis the helicity is invariant.

3. When a space reflection is applied, the sign of $\lambda$ is changed.

4. By applying the Lorentz transformation in the direction of momentum $\boldsymbol{p}$ the helicity remains unchanged, if the direction of $\boldsymbol{p}$ is not reversed.

\subsection{Kinematics}

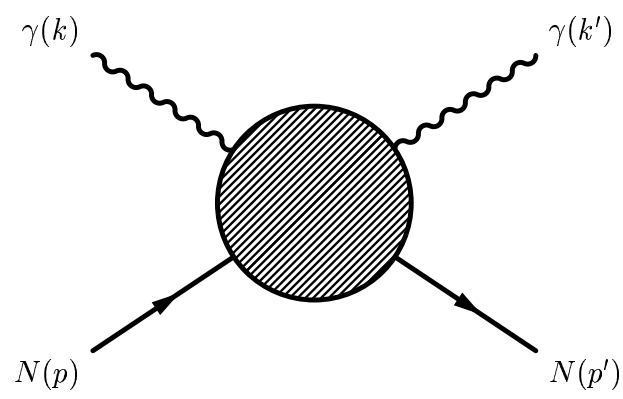

Figure 2.1.: The schematic view of the kinematics for the nucleon Compton scattering $\gamma N \longrightarrow \gamma N$.

For the elastic photon scattering by the nucleon in c.m.s.,

$$
\gamma(k)+N(p) \longrightarrow \gamma\left(k^{\prime}\right)+N\left(p^{\prime}\right),
$$

we define the kinematical variables $k=(\omega, \boldsymbol{k})$ and $k^{\prime}=\left(\omega, \boldsymbol{k}^{\prime}\right)$ as four-momentum of the incoming and the outgoing photon. $p=(E, \boldsymbol{p})$ and $p^{\prime}=\left(E, \boldsymbol{p}^{\prime}\right)$ denote the initial and the final four-momentum of the target nucleon, respectively. The schematic illustration of the kinematics for this process is given in Fig. 2.1.

The process of Eq. (2.3) can then be described by the transition amplitude between the inital and the final states $T_{f i} \equiv<f|T| i>$ which is related to the scattering matrix $S_{f i} \equiv<f|S| i>$ as follows:

$$
S_{f i}=\delta_{f i}+i(2 \pi)^{4} \delta^{4}\left(k^{\prime}+p^{\prime}-k-p\right) T_{f i}
$$

Because the $T_{f i}$ has to be invariant under the Lorentz transformation [67], it can be represented as the function of the invariant variables $\nu=(s-u) / 4 m$ and $t$, where the 
usual Mandelstam variables [66] $s=(k+p)^{2}, t=\left(k-k^{\prime}\right)^{2}$ and $u=\left(k-p^{\prime}\right)^{2}$ are in c.m. frame given as

$$
\begin{aligned}
s & =(\omega+E)^{2}=W_{t o t}^{2}, \\
t & =-2 \omega^{2}(1-\cos \theta)=-|\boldsymbol{q}|^{2}, \\
u & =m^{2}-2\left(\omega E+\omega^{2}\right) .
\end{aligned}
$$

Here, $m$ is the mass of the nucleon, and $\theta$ is the c.m. scattering angle between the initial and the final photon directions. In the center of mass system the variable $s$ is the square of the total energy $W_{\text {tot }}$ of the system, and $t$ is equal to the negative squared of the momentum transfer $\boldsymbol{q}=\boldsymbol{k}^{\prime}-\boldsymbol{k}$. The energy and the three-momentum of the nucleon are expressed in terms of $s$ as

$$
\boldsymbol{p}^{2}=\omega^{2}=\frac{\left(s-m^{2}\right)^{2}}{4 s}, \quad E^{2}=\frac{\left(s+m^{2}\right)^{2}}{4 s} .
$$

The conventional c.m. scattering amplitude $f_{c m}$ and the transition amplitude $T_{f i}$ are connected to each other by a phase space factor $\Gamma_{c m}$ as follows:

$$
f_{c m}=\Gamma_{c m} T_{f i}, \quad \Gamma_{c m}=\frac{1}{8 \pi W_{t o t}} .
$$

\subsection{Helicity amplitudes}

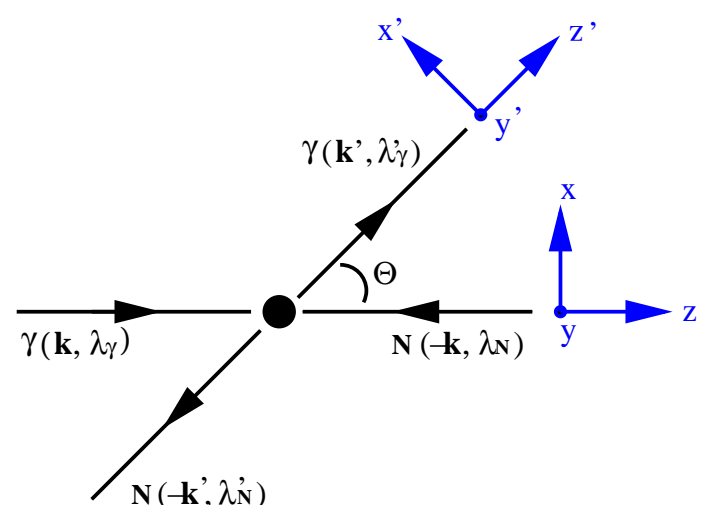

Figure 2.2.: The nucleon Compton scattering in c.m. frame

The incident photon moves along the positive $z$ direction, and the scattering takes placs in the $x z$ plane, as shown in Fig. 2.2. Assuming also that the spin of the photon and that of the nucleon are quantized along the directions of the photon momentum, $\hat{\boldsymbol{n}}=\boldsymbol{k} / \omega$ before the scattering and $\hat{\boldsymbol{n}}^{\prime}=\boldsymbol{k}^{\prime} / \omega$ after the scattering respectively. The 
transition matrix $T$ can then be described by the helicity amplitudes $T_{\lambda_{\gamma}^{\prime} \lambda_{N}^{\prime}, \lambda_{\gamma} \lambda_{N}}$ which are defined as

$$
T_{\lambda_{\gamma}^{\prime} \lambda_{N}^{\prime}, \lambda_{\gamma} \lambda_{N}} \equiv<k^{\prime} \lambda_{\gamma}^{\prime}, p^{\prime} \lambda_{N}^{\prime}|T| k \lambda_{\gamma}, p \lambda_{N}>
$$

Here, $\lambda_{\gamma}$ and $\lambda_{\gamma}^{\prime}$ refer to the initial and the final helicities of the photon, while $\lambda_{N}$ and $\lambda_{N}^{\prime}$ are those of the nucleon. Eq. (2.8) means that the helicity amplitudes are the transition amplitudes between the initial and the final states with fixed spin projections. Because of $\lambda_{\gamma}, \lambda_{\gamma}^{\prime}= \pm 1$ and $\lambda_{N}, \lambda_{N}^{\prime}= \pm 1 / 2$ there are sixteen helicity amplitudes. Using them, the $T$-matrix in the helicity space is represented as

$$
T=\left(\begin{array}{llll}
T_{11 / 2,11 / 2} & T_{11 / 2,1-1 / 2} & T_{11 / 2,-11 / 2} & T_{11 / 2,-1-1 / 2} \\
T_{1-1 / 2,11 / 2} & T_{1-1 / 2,1-1 / 2} & T_{1-1 / 2,-11 / 2} & T_{1-1 / 2,-1-1 / 2} \\
T_{-11 / 2,11 / 2} & T_{-11 / 2,1-1 / 2} & T_{-11 / 2,-11 / 2} & T_{-11 / 2,-1-1 / 2} \\
T_{-1-1 / 2,11 / 2} & T_{-1-1 / 2,1-1 / 2} & T_{-1-1 / 2,-11 / 2} & T_{-1-1 / 2,-1-1 / 2}
\end{array}\right) .
$$

Under the space inversion $P$ and the time reversal $T$ inversion,

$$
P: \lambda_{\gamma}, \lambda_{N} \rightarrow-\lambda_{\gamma},-\lambda_{N} \quad \text { and } \quad T: \lambda_{\gamma}, \lambda_{N} \rightarrow \lambda_{\gamma}^{\prime}, \lambda_{N}^{\prime},
$$

the helicity amplitudes satify [64, 55]

$$
\begin{aligned}
& T_{-\lambda_{\gamma}^{\prime}-\lambda_{N}^{\prime},-\lambda_{\gamma}-\lambda_{N}}=(-1)^{\lambda-\lambda^{\prime}} T_{\lambda_{\gamma}^{\prime} \lambda_{N}^{\prime}, \lambda_{\gamma} \lambda_{N}}, \\
& T_{\lambda_{\gamma}^{\prime} \lambda_{N}^{\prime}, \lambda_{\gamma} \lambda_{N}}=(-1)^{\lambda-\lambda^{\prime}} T_{\lambda_{\gamma} \lambda_{N}, \lambda_{\gamma}^{\prime} \lambda_{N}^{\prime}},
\end{aligned}
$$

with $\lambda^{\prime}=\lambda_{\gamma}^{\prime}-\lambda_{N}^{\prime}$ and $\lambda=\lambda_{\gamma}-\lambda_{N}$. The numbers of the independent amplitudes are thus reduced to six, here chosen to be [I] :

$$
\begin{array}{ll}
H_{1}=T_{11 / 2,11 / 2}, & H_{2}=T_{-11 / 2,-11 / 2}, \\
H_{3}=T_{1-1 / 2,11 / 2}, & H_{4}=T_{11 / 2,-11 / 2}, \\
H_{5}=T_{-1-1 / 2,11 / 2}, & H_{6}=T_{1-1 / 2,-11 / 2} .
\end{array}
$$

Both amplitudes $H_{1}$ and $H_{2}$ are related to the helicity non-flip transition states, whereas the amplitudes $H_{5}$ and $H_{6}$ are connected to double helicity flip. The $H_{3}$ and $H_{4}$ describe the process of the photon helicity flip without and with the nucleon helicity flip respectively. These different structures owe to the various spin-dependent interactions, i.e. spin-orbit, spin-spin and spin-tensor interactions [14]. By means of Eq. (2.12) the matrix $T$ reads

$$
T=\left(\begin{array}{rrrr}
H_{1} & -H_{3} & H_{4} & -H_{5} \\
H_{3} & H_{2} & H_{6} & H_{4} \\
H_{4} & -H_{6} & H_{2} & -H_{3} \\
H_{5} & H_{4} & H_{3} & H_{1}
\end{array}\right)
$$

It has to be noted that the diagonal elements of the matrix in Eq. (2.13) are made of the helicity non-flip amplitudes, while the double helicity flip waves are provided by the far off-diagonal elements. 


\subsubsection{Partial wave expansion}

As mentioned in section 2.1, the helicity states are invariant under any rotation. The inital states of the process of Eq. (2.3) can therefore be constructed by the states with the total angular momentum of the system $J$, their components along a specified $z$ direction $M=J_{z}$ and the net helicity $\lambda$. Analogously, the final states can be described by the states with $J^{\prime}, M^{\prime}=J_{z^{\prime}}^{\prime}$ and $\lambda^{\prime}$ :

$$
\begin{aligned}
\mid \hat{\boldsymbol{n}} \lambda_{\gamma} \lambda_{N}> & =\sum_{J, M}<J M \lambda_{\gamma} \lambda_{N}\left|\hat{\boldsymbol{n}} \lambda_{\gamma} \lambda_{N}>\right| J M \lambda_{\gamma} \lambda_{N}> \\
& =\sum_{J, M} D_{\lambda M}^{J}(\hat{\boldsymbol{n}}) \mid J M \lambda_{\gamma} \lambda_{N}>
\end{aligned}
$$

The Wigner function $D_{\lambda M}^{J}(\hat{\boldsymbol{n}})$ can be reduced under the $S U(2)$ to $d$-function, where the photon momentum direction $\hat{\boldsymbol{n}}$ is represented by two spherical angles $\phi$ and $\theta$ :

$$
D_{\lambda M}^{J}(\hat{\boldsymbol{n}})=\exp \{i M \phi\} d_{\lambda M}^{J}(\theta) .
$$

If the direction of the $\hat{\boldsymbol{n}}$ is taken as $z$-axis, then we can set $\phi=0$. With the help of the orthogonality relation of $d$-function,

$$
\int_{0}^{\pi} d_{\lambda^{\prime} M^{\prime}}^{J^{\prime}{ }^{*}}(\theta) d_{\lambda M}^{J}(\theta) \sin \theta d \theta=\frac{2}{2 J+1} \delta_{J^{\prime} J} \delta_{\lambda^{\prime} \lambda} \delta_{M^{\prime} M}
$$

the helicity amplitudes $T_{\lambda_{\gamma}^{\prime} \lambda_{N}^{\prime}, \lambda_{\gamma} \lambda_{N}}$ are decomposed into the partial waves defined as $T_{\lambda_{\gamma}^{\prime} \lambda_{N}^{\prime}, \lambda_{\gamma} \lambda_{N}}^{J}(s) \equiv<J M \lambda_{\gamma} \lambda_{N}|T(s)| J^{\prime} M^{\prime} \lambda_{\gamma}^{\prime} \lambda_{N}^{\prime}>$, i.e. the $T$-matrix elements in an angular momentum representation for a given $J, M$ and the corresponding rotation function $d_{\lambda^{\prime} \lambda}^{J}(\theta)$ :

$$
\begin{aligned}
T_{\lambda_{\gamma}^{\prime} \lambda_{N}^{\prime}, \lambda_{\gamma} \lambda_{N}}(s, \theta)= & \sum_{J, M}<\hat{\boldsymbol{n}}^{\prime} \lambda_{\gamma}^{\prime} \lambda_{N}^{\prime}\left|J M \lambda_{\gamma} \lambda_{N}><J M \lambda_{\gamma} \lambda_{N}\right| T(s) \mid J^{\prime} M^{\prime} \lambda_{\gamma}^{\prime} \lambda_{N}^{\prime}> \\
& *<J^{\prime} M^{\prime} \lambda_{\gamma}^{\prime} \lambda_{N}^{\prime} \mid \hat{\boldsymbol{n}} \lambda_{\gamma} \lambda_{N}> \\
= & 8 \pi \sqrt{s} \sum_{J}(2 J+1) T_{\lambda_{\gamma}^{\prime} \lambda_{N}^{\prime}, \lambda_{\gamma} \lambda_{N}}^{J}(s) d_{\lambda^{\prime} \lambda}^{J}(\theta)
\end{aligned}
$$

where $d_{\lambda^{\prime} \lambda}^{J}(\theta)$ is associated with Jacobi polynomial as like

$$
d_{\lambda^{\prime} \lambda}^{J}(\theta)= \pm \sqrt{\frac{(J+M) !(J-M) !}{(J+N) !(J-N) !}}\left[\cos \frac{\theta}{2}\right]^{\left|\lambda+\lambda^{\prime}\right|}\left[\sin \frac{\theta}{2}\right]^{\left|\lambda-\lambda^{\prime}\right|} P_{(J-M)}^{\left|\lambda-\lambda^{\prime}\right|,\left|\lambda+\lambda^{\prime}\right|}(\cos \theta)
$$

with

$$
\begin{aligned}
M & \equiv \text { maximum of }\left(|\lambda|,\left|\lambda^{\prime}\right|\right), \\
N & \equiv \text { minimum of }\left(|\lambda|,\left|\lambda^{\prime}\right|\right) .
\end{aligned}
$$


With the reduced helicity amplitudes $\tau_{i},(i=1 \ldots 6)$ defined as

$$
\tau_{i}=8 \pi \sqrt{s} \sum_{J}(2 J+1) T_{\lambda_{\gamma}^{\prime} \lambda_{N}^{\prime}, \lambda_{\gamma} \lambda_{N}}^{J}(s) P_{(J-M)}^{\left|\lambda-\lambda^{\prime}\right|,\left|\lambda+\lambda^{\prime}\right|}(\cos \theta)
$$

the amplitudes $T_{\lambda_{\gamma}^{\prime} \lambda_{N}^{\prime}, \lambda_{\gamma} \lambda_{N}}$ are expressed as

$$
T_{\lambda_{\gamma}^{\prime} \lambda_{N}^{\prime}, \lambda_{\gamma} \lambda_{N}}(s, \theta)=\left[\cos \frac{\theta}{2}\right]^{\left|\lambda+\lambda^{\prime}\right|}\left[\sin \frac{\theta}{2}\right]^{\left|\lambda-\lambda^{\prime}\right|} \tau_{i} .
$$

The $\tau_{i}$ are free from singularities owing to the factors $\cos (\theta / 2)^{\left|\lambda+\lambda^{\prime}\right|} \sin (\theta / 2)^{\left|\lambda-\lambda^{\prime}\right|}[57$, 87]. In concrete, the helicity amplitudes in terms of $\tau_{i}$ read as follows:

$$
\begin{array}{ll}
T_{11 / 2,11 / 2}=\cos ^{3} \frac{\theta}{2} \tau_{1}, & T_{-11 / 2,-11 / 2}=\cos \frac{\theta}{2} \tau_{2}, \\
T_{1-1 / 2,11 / 2}=\cos ^{2} \frac{\theta}{2} \sin \frac{\theta}{2} \tau_{3}, & T_{11 / 2,-11 / 2}=\cos \frac{\theta}{2} \sin ^{2} \frac{\theta}{2} \tau_{4}, \\
T_{-1-1 / 2,11 / 2}=\sin \frac{\theta}{2} \tau_{5}, & T_{1-1 / 2,-11 / 2}=\sin ^{3} \frac{\theta}{2} \tau_{6} .
\end{array}
$$

Due to the conservation of the total angular momentum

$$
\begin{array}{ll}
T_{\lambda_{\gamma}^{\prime} \lambda_{N}^{\prime}, \lambda_{\gamma} \lambda_{N}}(s, \theta) \propto t^{\left|\lambda-\lambda^{\prime}\right| / 2}, & \text { as } \theta \rightarrow 0 \\
T_{\lambda_{\gamma}^{\prime} \lambda_{N}^{\prime}, \lambda_{\gamma} \lambda_{N}}(s, \theta) \propto t^{\left|\lambda+\lambda^{\prime}\right| / 2}, & \text { as } \theta \rightarrow \pi
\end{array}
$$

must be satisfied at the forward and backward angle, respectively. That is, at $\theta=0$ only the amplitudes describing no helicity flip states are allowed, while at $\theta=\pi$ the amplitudes with double helicity flip are permitted.

\subsection{Parity conservation and multipoles}

By virtue of parity conservation only superpositions of the partial waves with opposite helicities have a definite parity [84]. These linear combinations of partial waves are called the electric and magnetic multipoles. In the notations of Ref. [ 8.5$]$ the multipoles of Compton scattering are represented as $f_{T \rightarrow T^{\prime}}^{J=L \pm 1 / 2} \equiv f_{T T^{\prime}}^{L \pm}$, where $J=L \pm 1 / 2$ and $T T^{\prime}$ stand for the total angular momentum of the $\gamma N$ system and the multipolarities of the initial and final photon, respectively. Because of $T T^{\prime}=M$ or $E$ there are such Compton multipoles as $f_{E E}^{L+}, f_{M M}^{L+}, f_{E E}^{(L+1)-}, f_{M M}^{(L+1)-}, f_{E M}^{L+}=f_{M E}^{(L+1)-}$ and $f_{E M}^{L-}=$ $f_{M E}^{(L-1)+}$. Here, the wave $f_{E M}^{L+}$ describes, for example, the transition between an electric $2^{L}$-pole in an initial state to a magnetic $2^{L^{\prime}}$-pole of the same parity $\left(L^{\prime}=L \pm 1\right)$ in a final state. The other waves have an analogous meaning. 


\subsubsection{Unitarity bound}

The unitarity condition for the partial waves says that the imaginary parts of the multipoles are given by the partial amplitudes of the intermediate states $\alpha$ :

$$
2 \operatorname{Im} T_{\lambda_{\gamma}^{\prime} \lambda_{N}^{\prime}, \lambda_{\gamma} \lambda_{N}}^{J}=q \sum_{\alpha} T_{\alpha, \lambda_{\gamma}^{\prime} \lambda_{N}^{\prime}}^{J *} T_{\alpha, \lambda_{\gamma} \lambda_{N}}^{J}
$$

where $q$ is the c.m. momentum of the intermediate particles. The sum is taken over the helicities and $J$. Therefore, the $\operatorname{Im} f_{T T^{\prime}}^{L \pm}$ can be expressed by the multipoles of pion photoproduction. In the region of the $\Delta$-resonance, single pion photoproduction $\gamma N \rightarrow \pi N$ provides the main contribution to the $\operatorname{Im} f_{T T^{\prime}}^{L \pm}$ in the form

$$
\begin{array}{ll}
\operatorname{Im} f_{E E}^{L \pm}=q \sum_{c}\left|E_{(L \pm 1) \mp}^{(c)}\right|^{2}, & \operatorname{Im} f_{M M}^{L \pm}=q \sum_{c}\left|M_{L \pm}^{(c)}\right|^{2}, \\
\operatorname{Im} f_{E M}^{L \pm}=q \sum_{c} \operatorname{Re}\left(E_{(L \pm 1) \mp}^{(c)} M_{(L \pm 1) \mp}^{(c) *}\right), & \operatorname{Im} f_{M E}^{L \pm}=q \sum_{c} \operatorname{Re}\left(E_{L \pm}^{(c)} M_{L \pm}^{(c) *}\right) .
\end{array}
$$

Here, the $E_{l \pm}$ and the $M_{l \pm}$ are the multipoles of single $\pi$-photoproduction in CGLN notation [49] with the parity $P=(-1)^{l+1}$ and the orbital momentum $l=L \pm 1$ of the $\pi N$-system. The sum is taken for both channels $\pi^{+} n$ and $\pi^{0} p$ in case of the proton, and $\pi^{-} p$ and $\pi^{0} n$ for the neutron.

The helicity amplitudes at $\omega=320 \mathrm{MeV}$ and their multipole decompositions $f_{T T^{\prime}}^{L \pm}$ with respect to energies up to $500 \mathrm{MeV}$ are illustrated numerically in Fig. 2.3 and 2.4, respectively. The solid and dashed lines present the real and imaginary parts of the amplitudes based on the pion amplitudes of the VPI group [73, 74], solution SAIDSP98K. On the other hand, the dotted curves for the Re $f_{T T^{\prime}}^{L \pm}$ and the dotted-dashed curves for the $\operatorname{Im} f_{T T^{\prime}}^{L \pm}$ in Fig. 2.4 result from the photoamplitudes of Ref. [866]. The upper six subfigures show the proton amplitudes, while the lower six one display the neutron amplitudes. At a given energy the $\operatorname{Im} T_{\lambda_{\gamma}^{\prime} \lambda_{N}^{\prime}, \lambda_{\gamma} \lambda_{N}}$ are predominating, see Fig. 2.3. Indeed, the imaginary parts of $T_{1-1 / 2,11 / 2}, T_{11 / 2,-11 / 2}$ and $T_{-1-1 / 2,11 / 2}$ have their maximum at the angles of around $\theta=\pi / 2$. At $\theta=0$ only the $T_{11 / 2,11 / 2}$ and $T_{-11 / 2,-11 / 2}$ are different from zero, whereas at the angle $\theta=\pi$ just the double spin-flip amplitudes $T_{-1-1 / 2,11 / 2}$ and $T_{1-1 / 2,-11 / 2}$ survive. This is, as mentioned in section 2.3, due to momentum conservation. Furthermore, the $T_{11 / 2,11 / 2}$ and $T_{1-1 / 2,-11 / 2}$ have larger values than the $T_{11 / 2,11 / 2}$ and $T_{1-1 / 2,-11 / 2}$ at this forward and backward angle, respectively.

In the region of $\Delta$, the dominant $f_{M M}^{1+}$ as well as the small $f_{M E}^{1+}$ show a typical resonance structure in which the corresponding real parts decrease rapidly with energies, see Fig. 2.4. In comparision with the two results obtained from the SAID solution and from Ref. [86] the difference appears mostly in the amplitudes $f_{E E}^{1-}$ and $f_{M E}^{1+}$, while in the amplitudes $f_{E E}^{1+}, f_{M M}^{1-}$ and $f_{E M}^{1+}$ the main difference is shown above pion threshold. As to $f_{M M}^{1+}$ both results agree quite well with each other. 

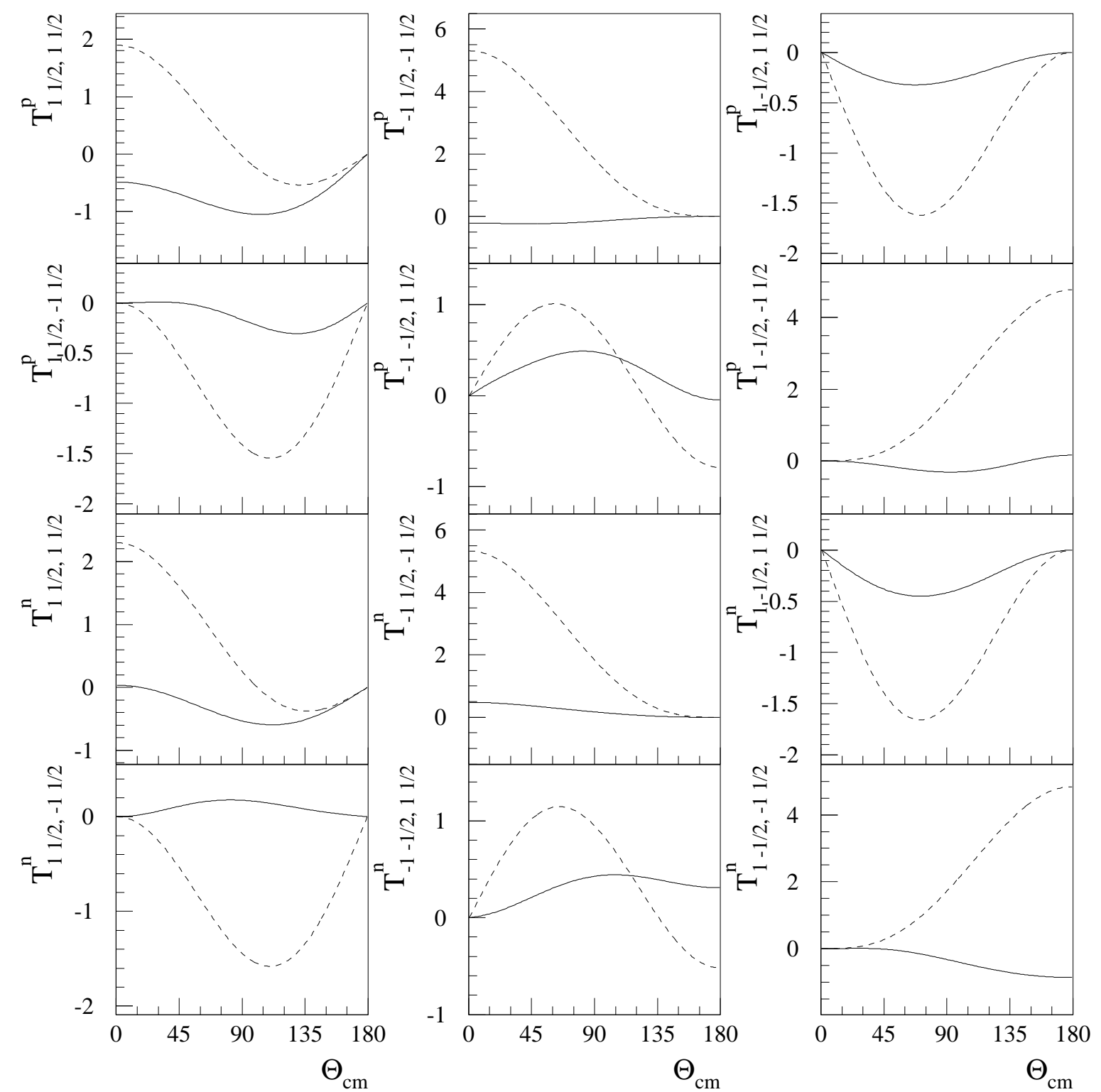

Figure 2.3.: The helicity amplitudes of the Compton scattering off the proton (upper subfigures) and the neutron (lower subfigures) at $\omega=320 \mathrm{MeV}$. The real parts of the amplitudes are plotted as the solid lines, while the dashed lines show the imaginary parts. 

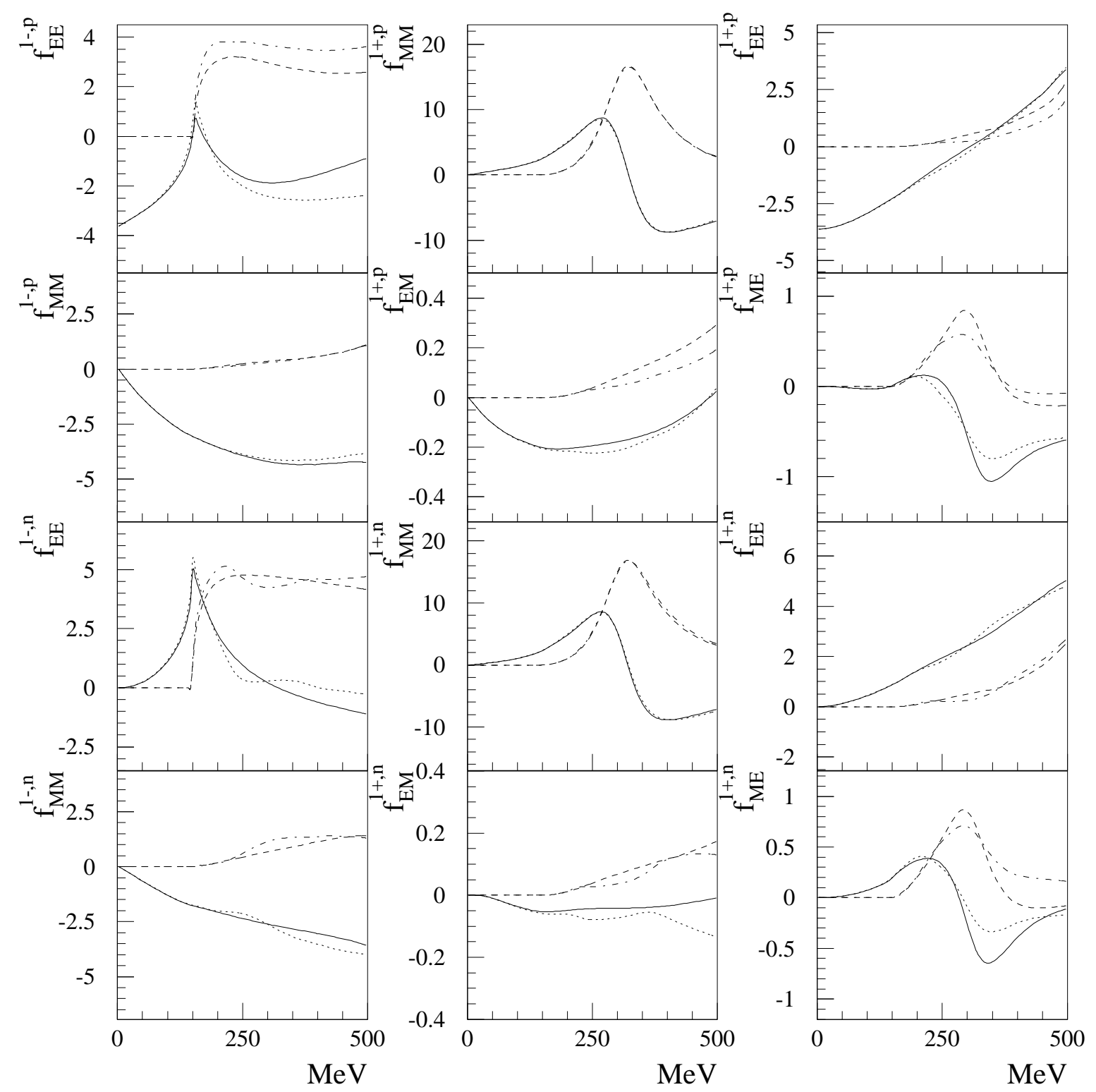

Figure 2.4.: The partial waves of Compton scattering off the proton (upper subfigures) and the neutron (lower subfigures) at the c.m. angle $\theta_{c m}=\pi$. The amplitudes based on the pion multipoles by the VPI group [73, 744] are presented by the solid (real parts) and dashed lines (imaginary parts) respectively. On the other hand, the results using multipoles in Ref. [86] are displayed with the dotted lines for the real parts and with the dotted-dashed lines for the imaginary parts. 


\subsection{Invariant amplitudes}

At an arbitary spin projection the Compton scattering amplitude $T$ may be expressed in terms of scalar invariant coefficients of a set of basis tensors in a vector space given by the appropriate products of the matrices and polarization vectors:

$$
T=\bar{u}^{\prime}\left(p^{\prime}\right) e^{\prime * \mu}\left(k^{\prime}\right) M_{\mu \nu} e^{\nu}(k) u(p) .
$$

Here, $u$ and $u^{\prime}$ are bispinors of the nucleon normalized as $\bar{u} u=2 m$, und $e$ and $e^{\prime}$ are photon polarization vectors. The tensor $M_{\mu \nu}$ may be expanded with respect to a tensor basis $I_{\mu \nu}^{i}$

$$
M_{\mu \nu}=\sum T_{i} I_{\mu \nu}^{i}
$$

where the coefficients of basis tensors, $T_{i}$, are called invariant amplitudes. $T_{i}$ are invariant functions of the two variables $\nu$ and $t$ as well as tensors $I_{\mu \nu}^{i}$. For an explicit construction of $I_{\mu \nu}^{i}$ four orthogonal vectors $P^{\prime}, K, N$ and $Q$ are defined by the 4momentum of the photon $k, k^{\prime}$ and by that of the nucleon $p, p^{\prime}$ [[7T]:

$$
\begin{aligned}
& P_{\mu}^{\prime}=P_{\mu}-K_{\mu} \frac{P K}{K^{2}}, \quad P=\frac{1}{2}\left(p+p^{\prime}\right), \quad K=\frac{1}{2}\left(k+k^{\prime}\right), \\
& N_{\mu}=\epsilon_{\mu \nu \alpha \beta} P^{\nu} Q^{\alpha} K^{\beta}, \quad Q=\frac{1}{2}\left(k^{\prime}-k\right)=\frac{1}{2}\left(p-p^{\prime}\right) .
\end{aligned}
$$

$\epsilon_{\mu \nu \alpha \beta}$ is an antisymmetric tensor and $\epsilon_{0123}=1$. The vectors $P^{\prime}, Q$ and $N$ are spacelike because of their orthogonality to the time-like vector $K\left(K^{2}=k k^{\prime}>0\right)$. The constraints of the Lorentz, gauge, $P$ and $T$ invariance lead to the following six independent covariants $I_{\mu \nu}^{i}$ :

$$
\begin{array}{ll}
\frac{P_{\mu}^{\prime} P_{\nu}^{\prime}}{P^{\prime 2}}, \quad \frac{P_{\mu}^{\prime} P_{\nu}^{\prime}}{P^{\prime 2}} \gamma K, & \frac{N_{\mu} N_{\nu}}{N^{2}}, \quad \frac{N_{\mu} N_{\nu}}{N^{2}} \gamma K, \\
\frac{P_{\mu}^{\prime} N_{\nu}-P_{\nu}^{\prime} N_{\mu}}{P^{\prime 2} K^{2}} i \gamma_{5}, & \frac{P_{\mu}^{\prime} N_{\nu}+P_{\nu}^{\prime} N_{\mu}}{P^{\prime 2} K^{2}} i \gamma_{5} \gamma K .
\end{array}
$$

In terms of the tensors of Eq. (2.30) the amplitude $T$ is expanded as

$$
\begin{aligned}
T=\bar{u}^{\prime}\left(p^{\prime}\right) e^{* \mu}\left(k^{\prime}\right) & \left\{-\frac{P_{\mu}^{\prime} P_{\nu}^{\prime}}{P^{\prime 2}}\left(T_{1}+\gamma K T_{2}\right)-\frac{N_{\mu} N_{\nu}}{N^{2}}\left(T_{3}+\gamma K T_{4}\right)\right. \\
& \left.+i \frac{P_{\mu}^{\prime} N_{\nu}-P_{\nu}^{\prime} N_{\mu}}{P^{\prime 2} K^{2}} \gamma_{5} T_{5}+i \frac{P_{\mu}^{\prime} N_{\nu}+P_{\nu}^{\prime} N_{\mu}}{P^{\prime 2} K^{2}} \gamma_{5} \gamma K T_{6}\right\} e^{\nu}(k) u(p),
\end{aligned}
$$

with

$$
\begin{aligned}
K^{2} & =-\frac{t}{4}=\frac{1}{8 s}\left(s-m^{2}\right)^{2}(1-\cos \theta), \\
P^{\prime 2} K^{2} & =\frac{1}{4}\left(s u-m^{4}\right)=-\frac{1}{8 s}\left(s-m^{2}\right)^{2}(1+\cos \theta), \\
N^{2} & =P^{\prime 2}\left(K^{2}\right)^{2} \sim \sin ^{2} \theta .
\end{aligned}
$$


By virtue of the crossing symmetry $s \leftrightarrow u$, i.e. $\nu \rightarrow-\nu, T_{i}$ satisfies the condition

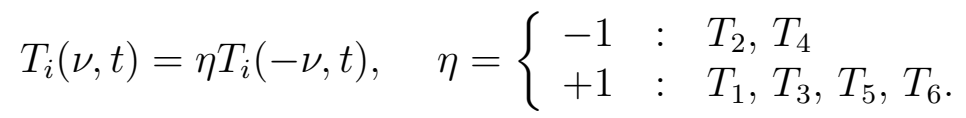

Hence, $T_{2}$ and $T_{4}$ are the odd functions of $\nu$, and the other amplitudes are the even functions.

\section{Analyticity}

$T_{i}$ are analytic functions of the complex variables $\nu$ and $t$ in the cut of $\nu t$ planes and have no kinematic singularitites [5], 5.9]. Aber they contain the kinematic zeros, which arise from the vanishing of denominators $P^{\prime}, K^{2}$ and $P^{\prime 2} K^{2}$ at the angles $\theta=0$ and $\pi$. To remove them the following linear combinations of $T_{i}$, that is $A_{i}$, are introduced $[62,69]$ :

$$
\begin{aligned}
& A_{1}=\frac{1}{t}\left[T_{1}+T_{3}+\nu\left(T_{2}+T_{4}\right)\right] \\
& A_{2}=\frac{1}{t}\left[2 T_{5}+\nu\left(T_{2}+T_{4}\right)\right] \\
& A_{3}=\frac{m^{2}}{m^{4}-s u}\left[T_{1}-T_{3}-\frac{t}{4 \nu}\left(T_{2}-T_{4}\right)\right], \\
& A_{4}=\frac{m^{2}}{m^{4}-s u}\left[2 m T_{6}-\frac{t}{4 \nu}\left(T_{2}-T_{4}\right)\right] \\
& A_{5}=\frac{1}{4 \nu}\left[T_{2}+T_{4}\right] \\
& A_{6}=\frac{1}{4 \nu}\left[T_{2}-T_{4}\right] .
\end{aligned}
$$

Eq. (2.34) means that the combination $T_{1}+T_{3}+\nu\left(T_{2}+T_{4}\right)$ has, for example, a kinematic zero at $K^{2} \sim t=0$. Therefore, the expression divided by $t$ has no additional constraints. Analogiously, the amplitudes $A_{3}$ and $A_{4}$ must vanish at the angle $\pi$ because of $\left(s u-m^{4}\right) \propto(1+\cos \theta)$. Indeed, the crossing symmetry of Eq. (2.33) results in $T_{2}=T_{4}=0$ at $\nu=0$, and then $A_{5}=A_{6}=0$ at $\nu=0$. As a result, the amplitudes $A_{i}$ are free from both kinematic singularities and kinematic constraints. The invariant amplitudes $A_{i}$ are also the analytic functions of $\nu$ and $t$ due to the analyticity of the amplitudes $T_{i}$. Noticed that all of $A_{i}$ are the even functions of $\nu$.

\subsection{Fixed- $t$ dispersion relation}

To calculate the amplitudes $A_{i}$ we start from the fixed- $t$ unsubtracted dispersion relation that are formulated by means of a Cauchy loop of finite size as [T]

$$
\operatorname{Re} A_{i}(\nu, t)=A_{i}^{B}(\nu, t)+A_{i}^{n B}(\nu, t)
$$




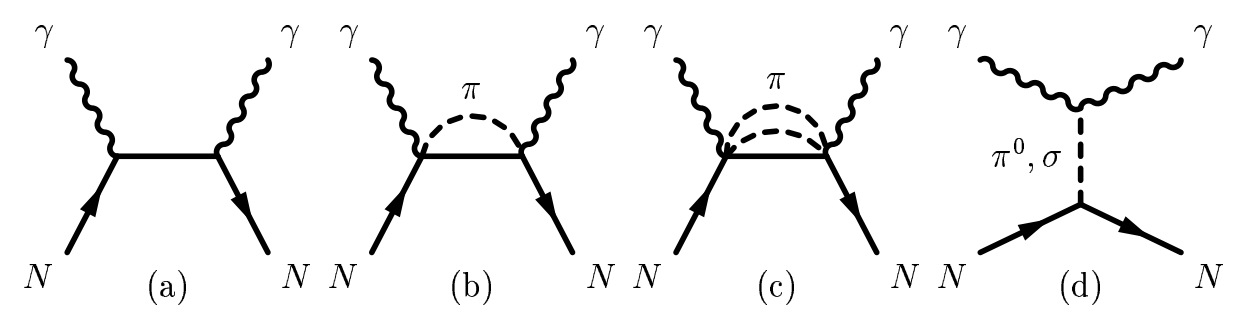

Figure 2.5.: The Feynman diagrams of the nucleon Compton scattering. In diagram (a) the Born contribution in the s-channel is represented. Diagrams (b) and $(c)$ show the $1 \pi$ - and $2 \pi$ intermediate states affecting on the integral part $A_{i}^{\text {int }}$ respectively. The $\pi^{0}$ and the $\sigma$-meson exchange diagram in the $\gamma \gamma$-channel is given in $(d)$, which is related to asymptotic part $A_{i}^{\text {as }}$ of dispersive calculation.

where $A_{i}^{B}$ and $A_{i}^{n B}$ denote the Born part and the non-Born part of the amplitudes respectively. The Feymann Diagrams related to the intermediates states of the nucleon Compton scattering are represented in Fig. 2.5.

The Born contribution $A_{i}^{B}$ describes the photon scattering off a rigid particle and has a purely pole form which is caused by single nucleon intermediate states in the $s$ - or the $u$-channel at zero energy, see the first diagram in Fig. 2.5. This term is determined by the electric charge $\tau=\left(1+\tau_{3}\right) / 2$ and the anomalous magnetic moment of the nucleon $\kappa=1.793 \tau-1.913(1-\tau)$ [4]:

$$
A_{i}^{B}(\nu, t)=\frac{a_{i}(t)}{\left(s-m^{2}\right)\left(u-m^{2}\right)}=\frac{r_{i}(t)}{t^{2}-16 m^{2} \nu^{2}},
$$

with

$$
\begin{gathered}
r_{1}=4 m e^{2}\left(-2 \tau+r_{3} \frac{t}{4 m^{2}}\right), \quad r_{2}=4 m e^{2}\left(2 \kappa \tau+2 \tau+r_{3} \frac{t}{4 m^{2}}\right), \\
r_{3}=r_{5}=4 m e^{2}\left(\kappa^{2}+2 \kappa \tau\right), \quad r_{4}=4 m e^{2} \kappa^{2}, \quad r_{6}=-4 m e^{2}\left(\tau^{2}+2 \kappa \tau+2 \tau\right) .
\end{gathered}
$$

Here $e^{2} \simeq 4 \pi / 137$ and $s u=m^{4}$.

\subsubsection{Non-Born contribution}

The part of non-Born contribution $A_{i}^{n B}$ can be, in turn, divided into the usual dispersion integral part $A_{i}^{\text {int }}$ and the asymptotic part $A_{i}^{a s}$ :

$$
A_{i}^{n B}(\nu, t)=A_{i}^{i n t}(\nu, t)+A_{i}^{a s}(\nu, t) .
$$




\section{$s$-channel contribution}

The integral part $A_{i}^{\text {int }}$ is evaluated by an integral from pion photoproduction threshold $\nu_{t h r}=\omega_{t h r}+t / 4 m$ with $\omega_{t h r} \simeq 150 \mathrm{MeV}$ to a finite upper limit $\nu_{\max }$, for which in an actual calculation $\omega_{\max }=\nu_{\max }-t / 4 m=1.5 \mathrm{GeV}$ is employed:

$$
A_{i}^{i n t}(\nu, t)=\frac{2}{\pi} P \int_{\nu_{t h r}(t)}^{\nu_{\max }(t)} \operatorname{Im} A_{i}\left(\nu^{\prime}, t\right) \frac{\nu^{\prime} d \nu^{\prime}}{\nu^{\prime 2}-\nu^{2}} .
$$

By virtue of the optical theorem and the unitarity relation $\operatorname{Im} A_{i}$ can be reduced by photo-meson amplitudes or the cross section of the photoabsorption taken from the experiments. In the region of energy considered here it is saturated mainly by the single $\pi$-meson amplitudes and be supplemented by the double pion contribution, whose amplitude is calculated in the framework of the simple model of Ref. [T].

\section{High energy behavior}

The information about behaviors of the amplitudes $A_{i}$ at energies $\nu \leq \nu_{\max }$ are carried by the asymptotic part $A_{i}^{a s}$ which are formally given by an integral over the upper semicircle of radius $\nu_{\max }$ in a complex plane:

$$
A_{i}^{a s}(\nu, t)=\frac{1}{\pi} \operatorname{Im} \int_{\nu^{\prime}=\nu_{\max }(t) e^{i \phi}, 0<\phi<\pi} A_{i}\left(\nu^{\prime}, t\right) \frac{\nu^{\prime} d \nu^{\prime}}{\nu^{\prime 2}-\nu^{2}}
$$

According to the Regge theory [64, 57] the amplitudes $A_{3}, A_{4}, A_{5}$ and $A_{6}$ are assumed to be proportional to $\nu^{\alpha(t)-2}$ with a Regge pole trajectory $\alpha(t) \leq 1$ and approach then zero at large $\nu$ and fixed $t$. Eq. (2.40) can then be recasted to dispersion integral evaluated from $\nu_{\max }(t)$ to $\infty$ :

$$
A_{i}^{a s}(\nu, t)=\frac{2}{\pi} \int_{\nu_{\max }(t)}^{\infty} \operatorname{Im} A_{i}\left(\nu^{\prime}, t\right) \frac{\nu^{\prime} d \nu^{\prime}}{\nu^{\prime 2}-\nu^{2}}, \quad(i=3,4,5,6) .
$$

The asymptotic part of $A_{3}+A_{6}$ at zero angle can be exactly estimated from the total photoabsorption cross section $\sigma_{\text {tot }}$ which reads in terms of the amplitudes $A_{3}$ and $A_{6}$ like

$$
\sigma_{\text {tot }}(\nu)=-2 \nu \operatorname{Im}\left\{A_{3}(\nu, 0)+A_{6}(\nu, 0)\right\} .
$$

it contributes about $6 \%$ to the sum of the electric and magnetic polarizabilities $\bar{\alpha}_{N}$ and $\bar{\beta}_{N}$ of the nucleon [22]:

$$
\begin{aligned}
\bar{\alpha}_{N}+\bar{\beta}_{N} & =\frac{1}{2 \pi^{2}} \int_{\nu_{t h r}}^{\infty} \sigma_{t o t}(\nu) \frac{d \nu}{\nu^{2}} \\
& =-\frac{1}{2 \pi}\left\{A_{3,6}^{i n t}(0,0)+A_{3,6}^{a s}(0,0)\right\}, \\
& \simeq 14.2 \times 10^{-4} \mathrm{fm}^{3}, \quad \text { for } \text { proton. }
\end{aligned}
$$


As to the two other amplitudes $A_{1}$ and $A_{2}$ which behave as $\sim \nu^{\alpha(t)}$ in the limit of $\nu \rightarrow \infty$ their high energy parts are replaced by a finite number of energy independent poles in $t$-channel. On the basis of good descriptions of all available data on the nucleon Compton scattering $A_{1}^{a s}$ and $A_{2}^{a s}$ are modeled by the $t$-channel exchange of

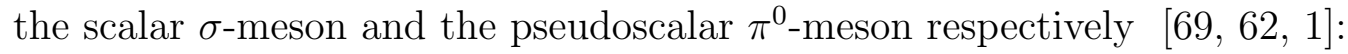

$$
A_{2}^{a s}(\nu, t) \approx A_{2}^{\pi^{0}}(t)=\frac{g_{\pi N N} F_{\pi^{0} \gamma \gamma}}{t-m_{\pi^{0}}^{2}} \tau_{3} F_{\pi}(t)
$$

where the product of coupling constants $g_{\pi N N} F_{\pi^{0} \gamma \gamma}=(-0.333 \pm 0.012) \mathrm{GeV}^{-1}$, and the form factor $F_{\pi}(t)$ at the moderate $t$ is given by

$$
F_{\pi}(t)=\frac{\Lambda_{\pi}^{2}-m_{\pi}^{2}}{\Lambda_{\pi}^{2}-t}
$$

with the cutoff parameter $\left.\Lambda_{\pi} \approx 1 \mathrm{GeV}[6]\right]$.

In the case of $\sigma$-exchange in $A_{1}$, a simpler form of

$$
A_{1}^{a s}(\nu, t) \approx A_{1}^{\sigma}(t)=\frac{g_{\sigma N N} F_{\sigma \gamma \gamma}}{t-m_{\sigma}^{2}}
$$

is applied, where the mass of $\sigma$-meson $m_{\sigma}$, which restricts the $t$-dependence of $A_{1}^{a s}(t)$, is phenomenologically given as $600 \mathrm{MeV}$. From the relation of $A_{1}$ with the electromagnetic polarizabilities

$$
A_{1}^{n B}(0,0)=A_{1}^{i n t}(0,0)+A_{1}^{a s}(0,0)=-2 \pi\left(\alpha_{N}-\beta_{N}\right)
$$

and using the previously calculated value of $A_{1}^{\text {int }}(0,0) \equiv\left(\alpha_{N}-\beta_{N}\right)^{\text {int }}$ the product of couplings constants $g_{\sigma N N} F_{\sigma \gamma \gamma}$ is extracted in a way of

$$
g_{\sigma N N} F_{\sigma \gamma \gamma}=2 \pi m_{\sigma}^{2}\left(\alpha_{N}-\beta_{N}\right)^{a s} .
$$

Fig. 2.6 display the real and the imaginary parts of the amplitudes $A_{i}$ at $\theta=30^{0}$ with solid and dotted lines respectively. The upper six figures are for the proton and the down six ones for the neutron. There are no considerable difference between the amplitudes $A_{i, p}$ and $A_{i, n}$ out of the real part of $A_{2}$ which has the opposite sign under the pion threshold.

Fig. 2.7 represe the contributions of two dominate pion multipoles $E_{0+}$ (dashed lines) and $M_{1+}$ (dotted lines) to $\operatorname{Im} A_{i}$ at the anlge $\theta=30^{0}$ and with respect to the energies $150 \leq \omega \leq 500$. The interference of these two multipoles results in the oscillations of the $\operatorname{Im} A_{i}$. 

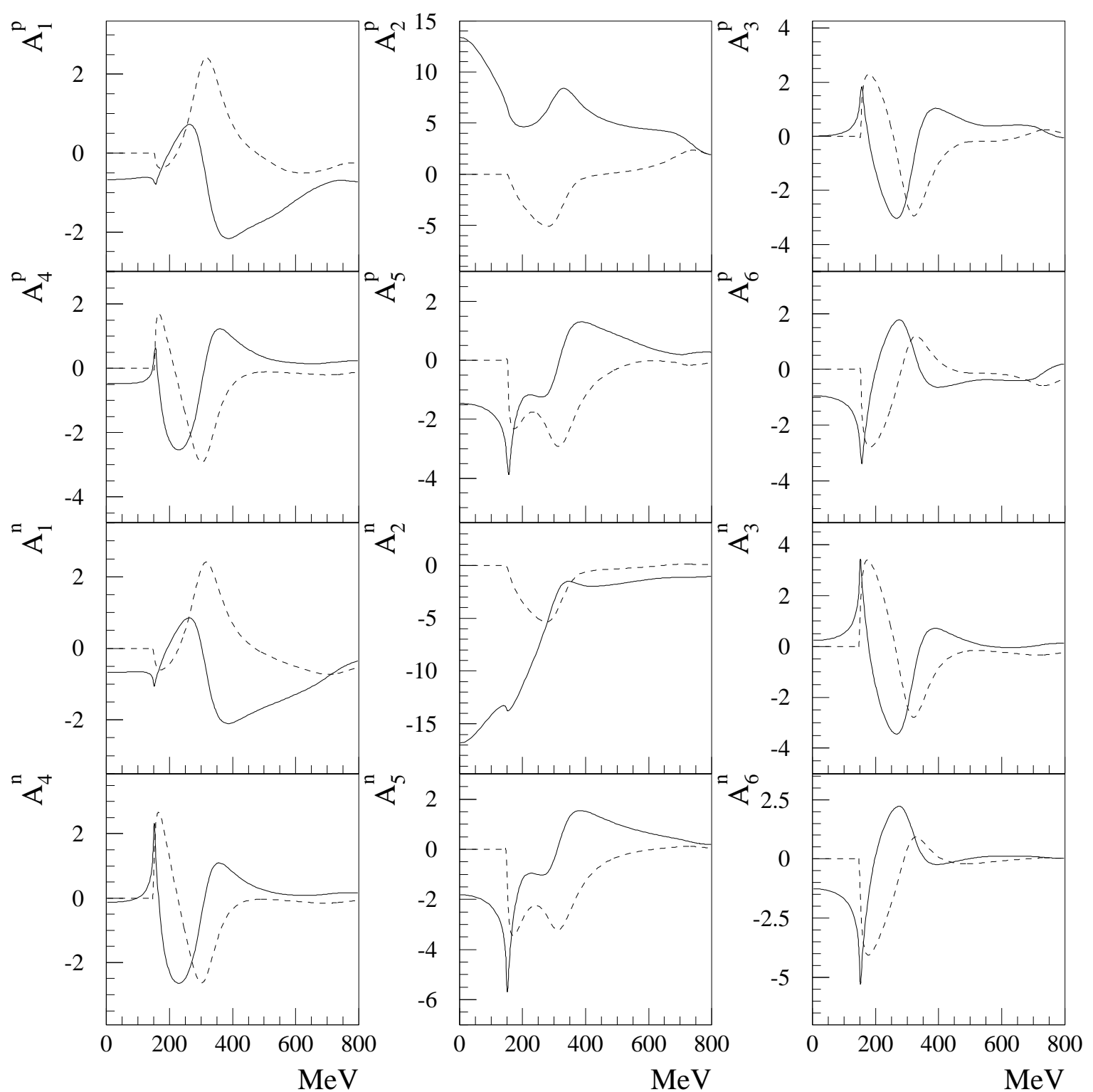

Figure 2.6.: The invariant amplitudes of the Compton scattering off the proton (upper) and neutron (low) at the c.m. angle $\theta_{c m}=30^{\circ}$. The real parts are represented with solid line and the imaginary parts with dashed line. 

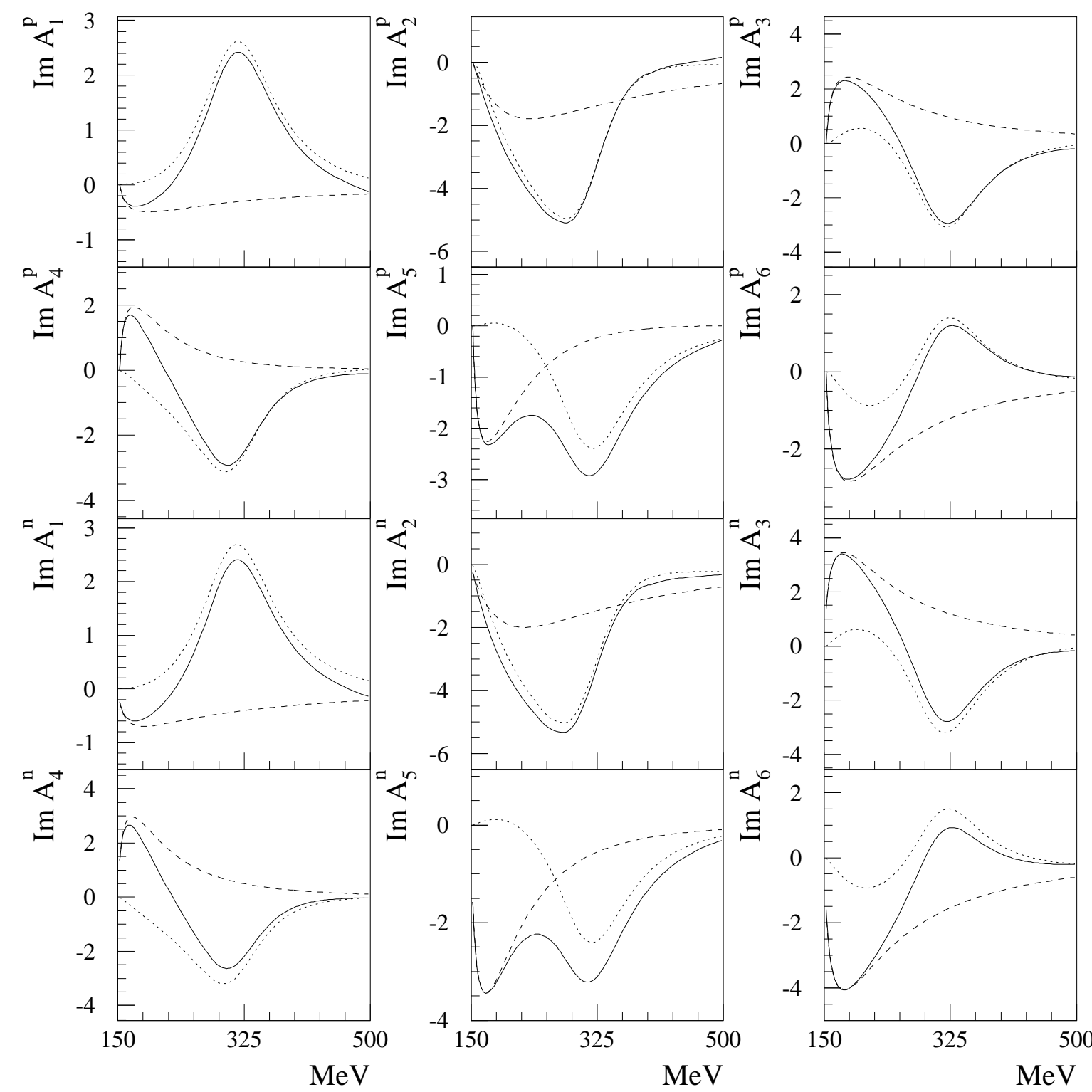

Figure 2.7.: The contributions of the pion multipoles $E_{0+}$ of dashed lines and $M_{1+}$ of dotted lines to the $\operatorname{Im} A_{i}$ of the proton (upper) and neutron (low). 


\section{Low energy theory}

Low energy amplitude of the nucleon Compton scattering can be described by the structure parameters such as the electric $\alpha_{N}$ and magnetic polarizabilities $\beta_{N}$ at the second order $O\left(\omega^{2}\right)$ in an expansion of the scattering amplitude as well as the four spin polarizabilities $\gamma_{E 1}, \gamma_{M 1}, \gamma_{E 2}$, and $\gamma_{M 2}$ first entered at the third order $O\left(\omega^{3}\right)$ $[58,75]$.

The quantities $\alpha$ and $\beta$ describe deformations of the constituent charge and magnetic moment distributions of the nucleon in the presence of a static eletromagnetic field. The current status of the electromagnetic polarizabilities of the nucleon has reported in [24, 2:3, 22: 3.5, 31, 3] :

$$
\begin{aligned}
& \alpha_{p}=12.1 \pm 0.8 \pm 0.5, \quad \beta_{p}=2.1 \mp 0.8 \mp 0.5, \\
& \alpha_{n}=12.6 \pm 1.5 \pm 2.0, \quad \beta_{n}=3.2 \mp 1.5 \mp 2.0
\end{aligned}
$$

in units of $10^{-4} \mathrm{fm}^{3}$. The first error is the combined statistical and systematic, and the second is due to the model dependence. These values have been confronted with various theoretical estimates such as non-relativistic and bag quark models [9, 10, 48, [77], the cloudy bag and soliton models [4.5, 78, 7.9].

The spin polarizabilities arise from the interaction of the photon field with constituent spin of the nucleon, and so are sensitive to the nucleon spin structure.

In contrast to $\alpha_{N}$ and $\beta_{N}$, the direct experimental determination of these spin polarizabilities has not yet been achieved. On the theoretical side, spin polarizabilities have been extracted from a dispersion relation using existing photoprodution multipoles taken from unpolarized experiments [15, 2, 5.3].

In this section we will evaluate the spin polarizabilities within the fixed- $t$ dispersion relation based on multipole analyses by VPI group and compare the resulting values with that of DR using another multipole of Ref. [68] and the theoretical investigation carried out within the Heavy Baryon Chiral Perturbation Theory (HBChPT).

\subsection{Spin polarizabilities}

Spin polarizabilities $\gamma_{i}$ characterize the spin-dependent part of the non-Born contribution to the scattering amplitude $T_{f i}^{n B, s p i n}$. We expand the scattering amplitude $T_{f i}=T_{f i}^{B}+T_{f i}^{n B}$ in power of $\nu^{2}$ and $t$. After the changing of the variables from $\nu^{2}$ 
and $t$ to $\omega$ and $\cos \theta$ we order every expanded terms in power of $\omega$. In center of mass system the part $T_{f i}^{n B, s p i n}$ can then be expressed by means of $\gamma_{i}$ as follows:

$$
\begin{gathered}
T_{f i}^{n B, s p i n}=8 \pi W_{t o t}\left\{i \omega^{3} \boldsymbol{\sigma} \cdot\left(\boldsymbol{e}^{* \prime} \times \boldsymbol{e}\right)\left(\gamma_{M 2}-\gamma_{E 1}\right)+i \omega^{3} \boldsymbol{\sigma} \cdot\left(\boldsymbol{s}^{* \prime} \times \boldsymbol{s}\right)\left(\gamma_{E 2}-\gamma_{M 1}\right)\right. \\
-i \omega^{3}\left(\boldsymbol{\sigma} \cdot \hat{\boldsymbol{k}} \boldsymbol{s}^{* \prime} \cdot \boldsymbol{e}-\boldsymbol{\sigma} \cdot \hat{\boldsymbol{k}}^{\prime} \boldsymbol{e}^{* \prime} \cdot \boldsymbol{s}\right) \gamma_{E 2} \\
\left.-i \omega^{3}\left(\boldsymbol{\sigma} \cdot \hat{\boldsymbol{k}} \boldsymbol{s}^{* \prime} \cdot \boldsymbol{e}-\boldsymbol{\sigma} \cdot \hat{\boldsymbol{k}} \boldsymbol{e}^{* \prime} \cdot \boldsymbol{s}\right) \gamma_{M 2}\right\}
\end{gathered}
$$

with the two magnetic vectors $s$ and $\boldsymbol{s}^{\prime}$ given as

$$
s=\hat{k} \times \boldsymbol{e}, \quad \boldsymbol{s}^{\prime}=\hat{\boldsymbol{k}}^{\prime} \times \boldsymbol{e}^{\prime} .
$$

From the relations of the coefficients $R_{i}$ of the spin basis in Eq. (3.2) to amplitudes $A_{i}$ listed in Appendix (E) the spin polarizabilites $\gamma_{E 1}, \gamma_{M 1}, \gamma_{E 2}$ and $\gamma_{M 2}$ are given via the following linear combinations of the low energy expansion of the non-Born part of the invariant amplitudes i.e. $a_{i}=A_{i}^{n B}(0,0)$ :

$$
\begin{aligned}
\gamma_{E 1} & =-\frac{1}{8 \pi m}\left[a_{2}+a_{4}-2 a_{5}-a_{6}\right], \\
\gamma_{M 1} & =-\frac{1}{8 \pi m}\left[a_{2}+a_{4}+2 a_{5}-a_{6}\right], \\
\gamma_{E 2} & =-\frac{1}{8 \pi m}\left[a_{2}+a_{4}+a_{6}\right], \\
\gamma_{M 2} & =-\frac{1}{8 \pi m}\left[-a_{2}+a_{4}+a_{6}\right] .
\end{aligned}
$$

The transparent physical meaning of $\gamma_{i}$ can be obvious by the multipole expansions of the amplitudes $R_{i}$, see in Appendix (E.6). By keeping only dipole-dipole and dipole-quadropole transition terms in Eq. (E.6) one retains then

$$
\begin{array}{rlrl}
\omega^{3} \gamma_{E 1} & =\left(f_{E E}^{1+}-f_{E E}^{1-}\right)^{n B}, & \omega^{3} \gamma_{M 1}=\left(f_{M M}^{1+}-f_{M M}^{1-}\right)^{n B}, \\
\omega^{3} \gamma_{E 2}=\left(6 f_{M E}^{1+}\right)^{n B}, & \omega^{3} \gamma_{M 2}=\left(6 f_{E M}^{1+}\right)^{n B} .
\end{array}
$$

As noticed in Eq. (3.5) the polarizabilities $\gamma_{E 1}$ and $\gamma_{M 1}$ represent spin-dependent transitions to the electric and magnetic dipole states respectively, while $\gamma_{E 2}$ and $\gamma_{M 2}$ describe the transitions of $M 1 \rightarrow E 2$ and $E 1 \rightarrow M 2$.

\section{Forward and backward spin polarizabilities}

In the case of the forward $\left(\hat{\boldsymbol{k}}^{\prime}=\hat{\boldsymbol{k}}\right)$ and the backward scattering $\left(\hat{\boldsymbol{k}}^{\prime}=-\hat{\boldsymbol{k}}\right)$, the amplitude of Eq. (3.2) reduces to

$$
\left[T_{f i}^{n B, s p i n}\right]_{\theta=0}=8 \pi W_{t o t} ;\left\{i \omega^{3} \gamma \boldsymbol{\sigma} \cdot\left(\boldsymbol{e}^{* \prime} \times \boldsymbol{e}\right)\right\}
$$


and

$$
\left[T_{f i}^{n B, s p i n}\right]_{\theta=\pi}=8 \pi W_{t o t} ;\left\{i \omega^{3} \delta \boldsymbol{\sigma} \cdot\left(\boldsymbol{e}^{* \prime} \times \boldsymbol{e}\right)\right\},
$$

where the forward $\gamma$ and backward $\delta$ spin- polarizability defined as the coefficients of $i \omega^{3} \boldsymbol{\sigma} \cdot\left(\boldsymbol{e}^{* \prime} \times \boldsymbol{e}\right)$ are

$$
\begin{aligned}
& \gamma=-\gamma_{E 1}-\gamma_{M 1}-\gamma_{E 2}-\gamma_{M 2}=\frac{1}{2 \pi m} a_{4} \\
& \delta=-\gamma_{E 1}+\gamma_{M 1}+\gamma_{E 2}-\gamma_{M 2}=-\frac{1}{2 \pi m}\left[a_{2}+a_{5}\right]
\end{aligned}
$$

\subsection{Sum rules}

As shown in Eqs. (3.4) and (3.8), the spin polarizabilities are determined by the nonBorn parts of the amplitudes $A_{2,4,5,6}$ at $\nu=t=0$. And the amplitudes at the low energy limit $a_{i}=A_{3,4,5,6}^{n B}(0,0)$ satisfy, as mentioned in section 2.5, an unsubtracted dispersion relation at fixed $t=0$ :

$$
a_{3,4,5,6}=\frac{2}{\pi} \int_{\nu_{t h r}(t)}^{\infty} \operatorname{Im} A_{i}\left(\nu^{\prime}, 0\right) \frac{\nu^{\prime} d \nu^{\prime}}{\nu^{\prime 2}-\nu^{2}} .
$$

which allow to evaluate forward spin polarizability $\gamma$ as well as linear combinations of polarizabilities $\gamma_{E 1}, \gamma_{M 1}, \gamma_{E 2}$ and $\gamma_{M 2}$ such as

$$
\begin{aligned}
\gamma_{E 1}+\gamma_{E 2} & =-\frac{1}{4 \pi m}\left[a_{4}-a_{5}\right], \\
\gamma_{M 1}+\gamma_{M 2} & =\frac{1}{4 \pi m}\left[a_{4}+a_{5}\right], \\
\gamma_{E 1}+\gamma_{M 1} & =-\frac{1}{4 \pi m}\left[a_{4}-a_{6}\right], \\
\gamma_{E 2}+\gamma_{M 2} & =-\frac{1}{4 \pi m}\left[a_{4}+a_{6}\right] .
\end{aligned}
$$

through the knowledge of photoproduction cross sections only. Of especial, via optical theorem found a sum-rule for a forward spin-polarizability in a form

$$
\gamma=\frac{1}{4 \pi^{2}} \int_{\nu_{t h r}}^{\infty} \frac{\sigma_{1 / 2}-\sigma_{3 / 2}}{\nu^{\prime 3}} d \nu^{\prime}
$$

which is originated from Gell-Mann, Goldberg and Thirring (GGT) [6.5]. Here $\sigma_{1 / 2,3 / 2}$ are the photoabsorption cross sections for parallel and anti-parallel alignments of photon and target helicities. 

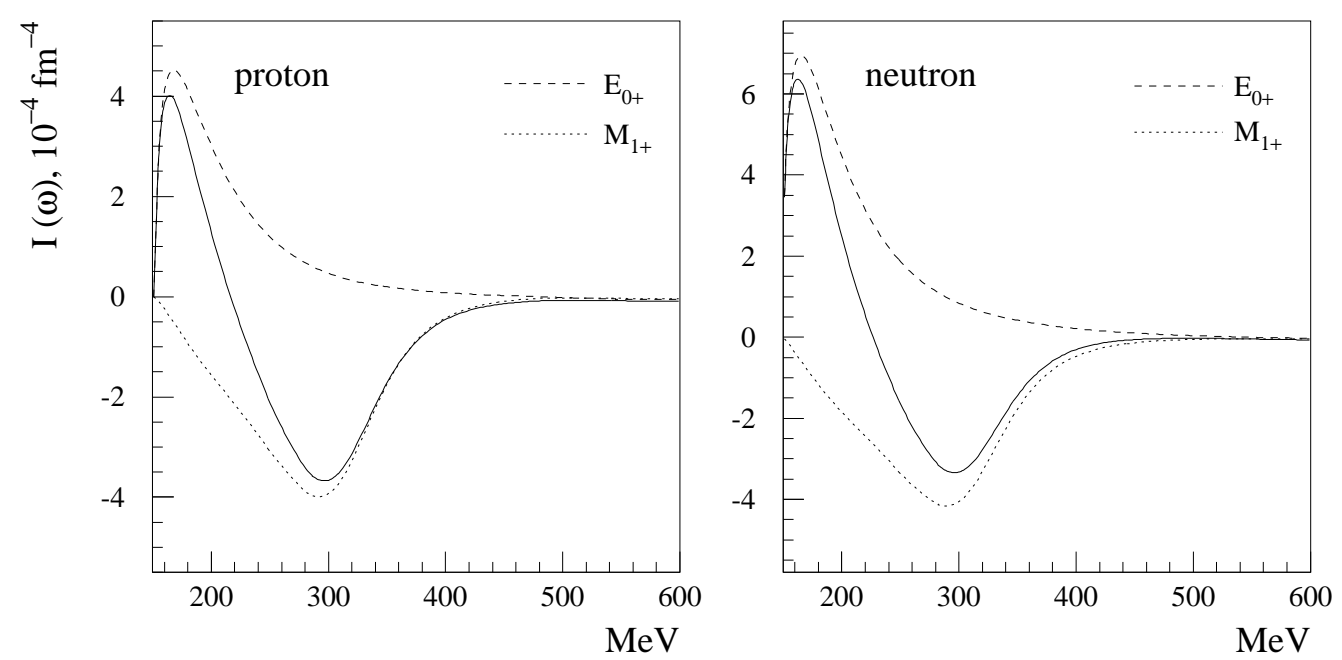

Figure 3.1.: The integrand of $\gamma=\int_{\nu_{t h r}}^{\infty} I(\nu) d \nu^{3}, I(\nu)=\Delta \sigma(\nu) / 4 \pi^{2} \nu^{3}$ for the proton (above) and neutron (under), where $\Delta \sigma(\nu)=\sigma_{1 / 2}-\sigma_{3 / 2}$. The dashed line represent the $E_{0+}$-contribution to $\gamma$, while the $M_{1+}$-contribution to $\gamma$ is plotted with dotted line.

In Fig. 3.1 the whole integrand in Eq. (3.10) are plotted with solid lines for the proton (left) and neutron (right). The intgrand shows its maximum and minimum at energies $\omega \simeq 150 \mathrm{MeV}$ and $\omega \simeq 300 \mathrm{MeV}$, respectively and practically fast vanishes above $\omega \simeq 500 \mathrm{MeV}$. It may be due to the damping factor $1 / \omega^{3}$ in the integrand. We have also plotted the contributions from $E_{0+}$ and $M_{1+}$ multipoles with dashed and dotted lines, respectively, which are by far the largest contributions to $\gamma$. It is noticed that there is large cancellation between the contributions from these two multipoles. Concretly, $\gamma$ yields

$$
\gamma= \begin{cases}+1.6\left(E_{0+}\right)-2.9\left(M_{1+} E_{1+}\right)-0.2(\text { rest })=-1.5 & \text { proton } \\ +2.8\left(E_{0+}\right)-3.1\left(M_{1+} E_{1+}\right)-0.1(\text { rest })=-0.4 & \text { neutron. }\end{cases}
$$

The term of rest refers to involving of all other multipole amplitudes.

\subsection{Comparision with theoretical predictions}

The nucleon spin polarizabilities calculated within fixed- $t$ dispersion relation by using the SAID solution VPI-SP98K as input for the single-pion photoamplitude are 
presented in Tables 3.1-3.3 and are compared with the theoretical predictions in the framework of HBChPT [ $[82,8.3,801,81]$. Table 3.1 shows seperately the contributions from one-pion $(\pi N)$ and two-pion $(2 \pi N)$ intermediate states in the $s$-channel together with the asymptotic part in the $t$-channel $A_{2}^{a s}(t)$. Table 3.2 and Table 3.3 give the forward and backward spin polarizability, $\gamma_{0}$ and $\gamma_{\pi}$, as well as the combinations of the spin polarizabilities of Eq. (3.10), which are independent of the amplitude $A_{2}$, respectively.

In the $s$-channel, the main contribution to the $\gamma_{i}$ comes from single $\pi$-production, whereas two-pion photoproduction gives rise to an only negligible effect to the $\gamma_{i}$, see Table 3.1. As to the single-pion channel, the result of Drechsel et al. [18] is also represented which is based on the pion multipoles of Hanstein, Drechsel and Tiator (HDT) [68]. There are no large differences in magnitiudes of the polarizabilitits with respect to the application of the two various inputs of multipoles. Nevertheless, the spin polarizabilities, especially the forward spin polarizability, from the multipoles by the VIP group show some difference from that obtained by HDT multipole amplitudes. This may be, as disscused in Ref. [18], due to the fact that in HDT the amplitude $A_{0+}\left(=E_{0+}\right)$ for charged pions near the pion threshold is larger than that of SAID. Furthermore, two different upper limits of the integral in Eq. (3.10) such as $\omega_{\max }=500 \mathrm{MeV}$ at HDT and $\omega_{\max }=1500 \mathrm{MeV}$ at SAID also play a role in this discrepancy.

The chiral perturbation expansion to leading order (LO) $O\left(p^{3}\right)$ is determined by the graphs of the effective chiral Lagrangian with one-pion loop $(\pi N)$ and the $t$ channel $\pi^{0}$-exchange $\left(\pi^{0}\right)$, where the latter part is in accordance with the high-energy contribution $A_{2}^{a s}$ in DR (see Table 3.1). The $p$ denotes non-relativistic momenta. The proton and neutron forward spin polarizability from relativistic ChPT yielding $\gamma_{0}^{p, L O}=\gamma_{0}^{n, L O}=+4.6$ disagree dramatically with our values of $\gamma_{0}^{p}=-1.5$ and $\gamma_{0}^{n}=-0.4$. This disagreement is dimished by including correction terms such as $\Delta$-isobar excitation, which is especially important for the low-energy phenomena of the polarizabilities because of the relatively small nucleon-delta mass spliting $\Delta=m_{\Delta}-m_{N} \simeq 2 m_{\pi}$ and a large coupling to the $\pi N$-channel. In heavy baryon ChPT the $\Delta$-pole is thus introduced as an explicit degree of freedom, and the chiral expansion is taken to third order in a small energy scale $\epsilon=\left(m_{\pi}, \Delta\right)$, which contains any $O\left(p^{3}\right)$ result in Ref. [82] plus additional terms involving the delta resonance. Not only the $\Delta$-isobar excitation but also the $\Delta \pi$-loop are kept to the same order in this expansion. Numerically, however, their contribution to the $\gamma_{i}$ is, as shown in Table [3.1, ignorable.

The $\Delta$-pole contribution to the magnetic dipole spin polarizability $\gamma_{M 1}^{\Delta}$, given by

$$
\gamma_{M 1}^{\Delta}=\frac{\mu_{\pi N \Delta}^{2}}{4 \pi \Delta^{2}}
$$


is deduced to $+2.4[5,87]$ or to $+4.0[\underline{80}]$ depending on the values for the transition magnetic moment $\mu_{\pi N \Delta}$. In Table 3.1 we give the results with $\gamma_{M 1}^{\Delta}=+4.0$ as well as the $\Delta$-pole contribution to $\gamma_{E 2}^{\Delta}$. The sum of the $\pi N$-loop in LO and SLO term in HBChPT can be compared with the integral contribution in DR. In most cases, the $\gamma_{i}$ within HBChPT are fairly similar to our results. Nevertheless, our finding of $\gamma_{E 1}^{p}=-3.7$ evidently deviats from $\gamma_{E 1}^{p}=-5.8$ predicted by HBChPT. Indeed, $\gamma_{M 2}^{p, n}$ have opposite signs to that of a chiral expansion. These may account for the variousity of the proton forward spin polarizabilty as well as for the discrepancy of $\gamma_{E 1}+\gamma_{M 1}$ and $\gamma_{E 1}+\gamma_{E 2}$, see Tables 3.2 and 3.3. The values of the backward spin polarizability following from DR and HBChPT are close to each other by virtue of the dominant contribution of the $t$-channel with the exception of our somewhat larger non- $\pi^{0}$ part of $\gamma_{\pi}^{n}$ compared with that of HBChPT.

There have been two recent determinations of the proton backward spin polarizability of $\gamma_{\pi \text {,exp }}^{p}=-36.8 \pm 0.6_{\text {stat. }+ \text { syst. }} \pm 2.0_{\text {mod. }}$ obtained from the LARA experiment of Ref. [2.9] using the data between $200 \mathrm{MeV} \lesssim \omega \lesssim 800 \mathrm{MeV}$ and $30^{\circ} \lesssim \theta_{\text {lab }} \lesssim 150^{\circ}$ and $\gamma_{\pi, \exp }^{p}=-36.1 \pm 2.1_{\text {stat. }} \mp 0.4_{\text {syst. }} \pm 0.8_{\text {mod. }}$ from the TAPS measurement [20] with energies between $55 \mathrm{MeV}$ and $165 \mathrm{MeV}$ and angles $59^{0} \lesssim \theta_{l a b} \lesssim 155^{0}$. The indices stat and sys denote the statistical and systematic error, respectively. And mod refers to the model-dependent one. These extracted $\gamma_{\pi, \exp }^{p}$ are in quite good accordance with our result presented here. On the other hand, $\gamma_{\pi, \text { exp }}^{p}=-27.1 \pm 2.2_{\text {stat. }+ \text { syst. }} \pm 2.6_{\text {mod }}$ deduced from the analysis of the LEGS group [2, 5.3] using data up to the $2 \pi$ threshold $(309 \mathrm{MeV})$ is appreciably different to our calculation for $\gamma_{\pi}^{p}$, and in particular, the non$\pi^{0}$ part of $\gamma_{\pi, \exp }^{p}$ yielding $\gamma_{\pi, \exp }^{p, \text { non }-\pi^{0}}=17.9 \pm 3.4$ is approximately twice as large as the theoretical prediction $\gamma_{\pi, \text { the }}^{p, \pi^{0}} \simeq+8$. 
Table 3.1.: Separate contributions to the spin polarizabilities calculated within DR using SAID solution [73, 74] in comparision with the results of Ref. [18] and the predictions of HBChPT [8T]. The contribution from one-pion photoproduction $(\pi N)$ in DR corresponds to the sum of $\pi N$-loop $(\pi N)$ in leading order (LO) and the $\Delta$-pole term $(\Delta)$ in subleading order (SLO) of HBChPT. On the other hand, the contribution from two-pion photoproduction $(2 \pi N)$ in DR can be compared with the term of $\Delta \pi$-loop $(\Delta \pi)$ in HBChPT. The asymptotic parts in the $t$-channel $\left(A_{2}^{a s}(t)\right)$ of $\mathrm{DR}$ are comparable to the $t$-channel $\pi^{0}$-exchange $\left(\pi^{0}\right)$ of HBChPT. (All results are in units of $\left.10^{-4} \mathrm{fm}^{4}\right)$.

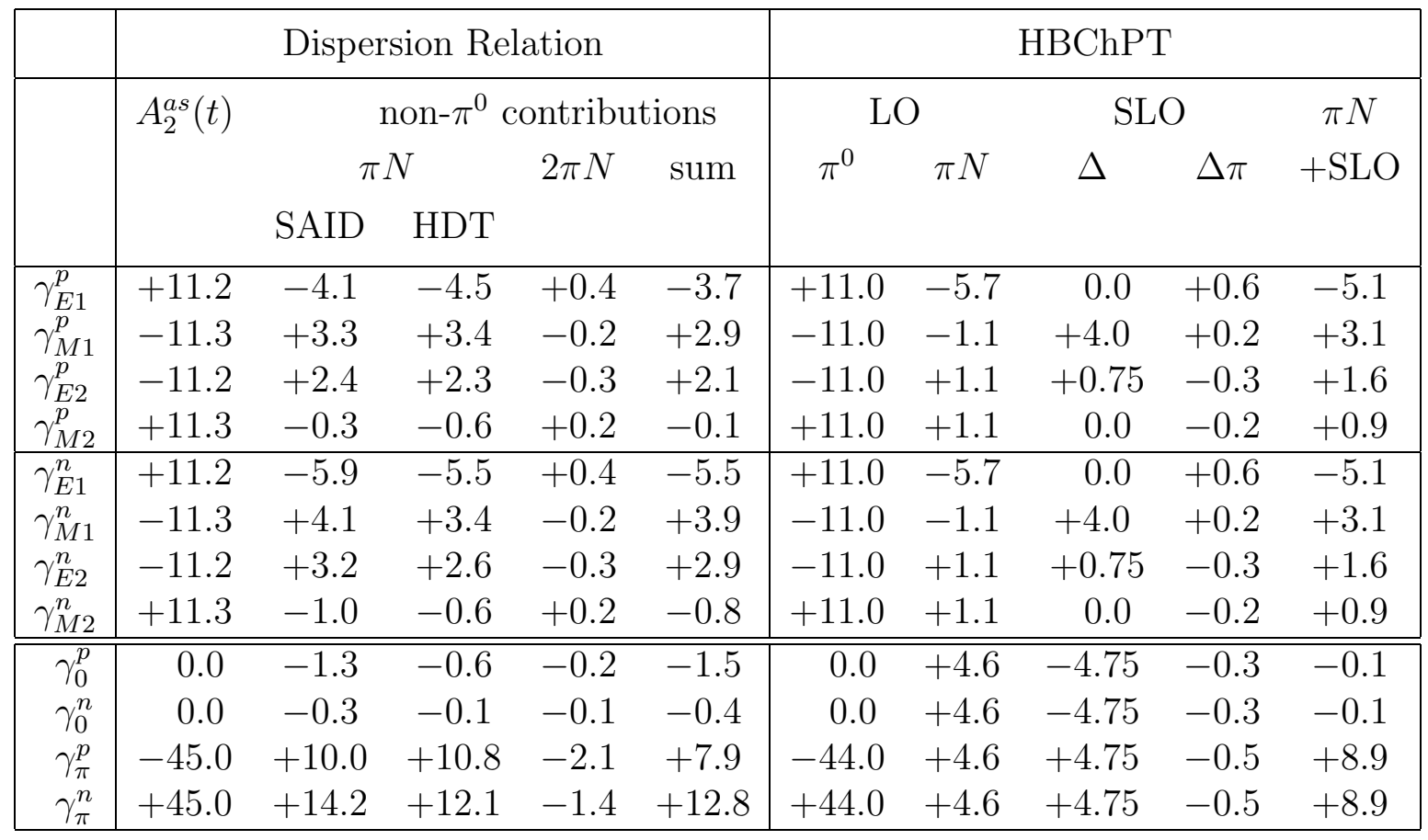


Table 3.2.: The results for the forward $\gamma_{0}$ and backward $\gamma_{\pi}$ spin polarizabilities of the nucleon in the framework of DR (SAID), DR (HDT) and HBChPT. (all results are in units of $\left.10^{-4} \mathrm{fm}^{4}\right)$.

\begin{tabular}{|c|c|c|c|c|c|c|}
\hline & \multicolumn{3}{|c|}{ Proton } & \multicolumn{3}{|c|}{ Neutron } \\
\hline & SAID & HDT & HBChPT & SAID & HDT & HBChPT \\
\hline$\gamma_{0}$ & -1.5 & -0.6 & -0.5 & -0.4 & -0.1 & -0.5 \\
\hline$\gamma_{\pi}$ & -37.1 & -34.2 & -35.1 & +57.8 & +57.1 & +52.9 \\
\hline
\end{tabular}

Table 3.3.: Linear combinations of the nucleon spin polarizabilities which are not affected by the asymptotic part of the amplitude $A_{2}$. (all results are in units of $\left.10^{-4} \mathrm{fm}^{4}\right)$.

\begin{tabular}{|c||cc|cc|c|}
\hline \multicolumn{1}{|c||}{} & \multicolumn{2}{c|}{ SAID } & \multicolumn{2}{c|}{ HDT } & HBChPT \\
$\gamma_{i}$ & proton & neutron & proton & neutron & \\
\hline \hline$\gamma_{E 1}+\gamma_{M 1}$ & -0.7 & -1.8 & -1.1 & -2.1 & -4.0 \\
$\gamma_{E 2}+\gamma_{M 2}$ & +2.2 & +2.2 & +1.7 & +2.0 & +2.0 \\
$\gamma_{E 1}+\gamma_{E 2}$ & -1.5 & -2.7 & -2.2 & -2.9 & -4.4 \\
$\gamma_{M 1}+\gamma_{M 2}$ & +3.0 & +3.1 & +2.8 & +2.8 & +2.4 \\
\hline
\end{tabular}




\section{Polarized nucleon Compton scattering}

In the experiments of Refs. [2.9, 20, 2, 5.3], the proton spin polarizability has been determined indirectly from an extrapolation of unpolarized Compton scattering. As shown in Eq. (3.10), the full set of $\gamma_{i}$ can however be extracted by polarized Compton scattering off the polarized target.

The combination of polarized photon beams with a polarized target also opens the possibilities for new approaches to small amplitudes, which often contain interesting information on subtle dynamical effects. Without polarization, a cross section is given only by the incoherent sum of the squares of reaction matrix elements, and small amplitudes are thus masked by the dominant ones. But, the polarization observables in general include interference terms of the various matrix elements in different ways. The small amplitudes may thus be considerably amplified by the interference with dominant matrix elements, and then polarization observables are sensitive to the strength of the small amplitudes.

In the present work, the strength of the resonant electric quadrupole component (E2) in the $\gamma N \rightarrow \Delta$ transition is of special interest which might only be a few percent of the dominant magnetic dipole transition (M1). This E2 component suggests in most nucleon models the deformation of the nucleon in its ground state, or in its first-excited state, i.e. the $P_{33}(1232)$ resonance, or in both, and therefore carries important information on the nucleon structure. The determination of the E2 component in the region of $\Delta$ is particularly complicated due to the presence of interfering processes, termed background contributions. These background processes give rise to the additional quadrupole amplitude which does not fulfill the requirement of unitarity (Fermi-Watson theorem). Unfortunately, both multipoles $M 1$ and E2, in particular the amplitude $E 2$, contain nonnegligable background contributions, and the background-resonance decomposition is very model-dependent [60] .

Further insight into the electric quadrupole admixture of the $N$ to $\Delta$ transition could be obtained by a precise determination of the resonant $E 2 / M 1$-ratio. In most nucleon models, the predictions for the value of $E 2 / M 1$ display considerable differences. For example, the constituent quark model predicts the values of $E 2 / M 1$ from $-0.1 \%$ up to $-1.2 \%$ [36, 17, 9, 12, 3.9], whereas the Skyrme model yields the largest negative values between $-2 \%$ and $-5 \%$ [5, 8.9]. The predictions of chiral-bag models [56, 70]], 
a quark model that includes two-body currents [6] and other models [3:3, 88, 37] range between $-0.9 \%$ and $-3.5 \%$.

In this chapter, we will demonstrate numerically the dependence of the observables on both quantitites of the polarizabilities and the E2/M1-ratio. Due to the convenience of the calculations, the observables are at first given in terms of helicity amplitudes and are in turn represented either by invariant amplitudes for an investigation of the response on the $\gamma_{i}$, or by multipoles in order to study the sensitivity to the mixing ratio, respectively.

\subsection{Polarization matrix}

\subsubsection{Photon}

The polarization state of a photon with a momentum $k$ is described by a polarization vector $e=(0, \boldsymbol{e})$ that satisfies the transversality condition $k e=0$. In a right-handed $x y z$-frame, where a photon moves along the $z$ direction, the three-polarization vector $\boldsymbol{e}$ can be written as a linear combination of either two mutually perpendicular linear polarizations $\boldsymbol{e}_{x}, \boldsymbol{e}_{y}$ or the right-hand and left-hand circular polarizations $\boldsymbol{e}_{+}, \boldsymbol{e}_{-}$:

$$
\begin{aligned}
\boldsymbol{e} & =e_{x} \boldsymbol{e}_{x}+e_{y} \boldsymbol{e}_{y}, \\
& =e_{+} \boldsymbol{e}_{+}+e_{-} \boldsymbol{e}_{-},
\end{aligned}
$$

where

$$
\boldsymbol{e}_{ \pm}=\mp \frac{1}{\sqrt{2}}\left(\boldsymbol{e}_{x} \pm i \boldsymbol{e}_{y}\right)
$$

The squares of the coefficients $e_{x}\left(e_{+}\right)$and $e_{y}\left(e_{-}\right)$determine the probabilities with which a photon has the polarization $\boldsymbol{e}_{x}\left(\boldsymbol{e}_{+}\right)$and $\boldsymbol{e}_{y}\left(\boldsymbol{e}_{-}\right)$, respectively. A partially polarized state of a photon is better specified by a density matrix $\rho_{\gamma} \equiv \boldsymbol{e} \boldsymbol{e}^{*}$ that can be expressed by means of the Stokes vector $\boldsymbol{\xi}=\left(\xi_{1}, \xi_{2}, \xi_{3}\right)$ [54, 76]]:

$$
\begin{aligned}
\rho_{\gamma} & =\frac{1}{2}(\mathbf{1}+\boldsymbol{\xi} \cdot \boldsymbol{\sigma}) \\
& =\frac{1}{2}\left(\begin{array}{cc}
1+\xi_{3} & \xi_{1}-i \xi_{2} \\
\xi_{1}+i \xi_{2} & 1-\xi_{3}
\end{array}\right),
\end{aligned}
$$

where 1 is the $2 \times 2$ unit matrix, and the three components of the Pauli matrix $\boldsymbol{\sigma}=\left(\sigma_{x}, \sigma_{y}, \sigma_{z}\right)$ are

$$
\sigma_{x}=\left(\begin{array}{rr}
0 & 1 \\
1 & 0
\end{array}\right), \quad \sigma_{y}=\left(\begin{array}{rr}
0 & -i \\
i & 0
\end{array}\right), \quad \sigma_{z}=\left(\begin{array}{rr}
1 & 0 \\
0 & -1
\end{array}\right) .
$$


Using Eqs. (4.1) and (4.2) the parameters $\xi_{i}$ are described as

$$
\begin{aligned}
& \xi_{1}=2 \operatorname{Re}\left(e_{x} e_{y}^{*}\right)=2 \operatorname{Im}\left(e_{+} e_{-}^{*}\right), \\
& \xi_{2}=2 \operatorname{Im}\left(e_{x} e_{y}^{*}\right)=-\left(\left|e_{+}\right|^{2}-\left|e_{-}\right|^{2}\right), \\
& \xi_{3}=\left(\left|e_{x}\right|^{2}-\left|e_{y}\right|^{2}\right)=-2 \operatorname{Re}\left(e_{+} e_{-}^{*}\right) .
\end{aligned}
$$

The parameter $\xi_{2}$ represents the degree of the circular polarization. In the case of a right and left circular polarization $\xi_{2}$ has values of +1 and -1 , respectively, and $\xi_{2}=$ \pm 1 corresponds to the helicity states $\lambda_{\gamma}= \pm 1$. The degree of the linear polarization is given by the parameter $\xi_{l}=\sqrt{\xi_{1}^{2}+\xi_{3}^{2}}$ with

$$
\xi_{3}=\xi_{l} \cos 2 \varphi, \quad \xi_{1}=\xi_{l} \sin 2 \varphi .
$$

Here, $\varphi$ denotes the angle between the electric field and the scattering plane. Namely, the parameter $\xi_{3}= \pm 1$ describes the degree of the linear polarization either parallel $(\varphi=0)$ or perpendicular $(\varphi=\pi / 2)$ to the scattering plane, and $\xi_{1}= \pm 1$ refers to the degree of the linear polarization at angle $\varphi= \pm \pi / 4$ with respect to the scattering plane.

All three Stokes parameters $\xi_{i}$ take values between -1 and 1, especially for an unpolarized state $\xi_{1}=\xi_{2}=\xi_{3}=0$. The total degree of the polarization is given by $|\xi|=\sqrt{\xi_{1}^{2}+\xi_{2}^{2}+\xi_{3}^{2}}$, which is $|\xi|<1$ in a mixed state and $|\xi|=1$ in a completely polarized case.

With respect to a coordinate system chosen as

$$
z\left(z^{\prime}\right)=\boldsymbol{k}\left(\boldsymbol{k}^{\prime}\right), \quad y=\boldsymbol{k} \times \boldsymbol{k}^{\prime}, \quad x\left(x^{\prime}\right)=\boldsymbol{k} \times \boldsymbol{k}^{\prime} \times \boldsymbol{k}\left(\boldsymbol{k}^{\prime}\right),
$$

Stokes parameters transform under the parity as [15]

$$
\xi_{1} \stackrel{P}{\longrightarrow}-\xi_{1}, \quad \xi_{2} \stackrel{P}{\longrightarrow}-\xi_{2}, \quad \xi_{3} \stackrel{P}{\longrightarrow} \xi_{3},
$$

under the time inversion $\left(\boldsymbol{k} \rightarrow-\boldsymbol{k}, \boldsymbol{e} \rightarrow-\boldsymbol{e}^{*}\right)$ as

$$
\xi_{1} \stackrel{T}{\longrightarrow}-\xi_{1}, \quad \xi_{2} \stackrel{T}{\longrightarrow} \xi_{2}, \quad \xi_{3} \stackrel{T}{\longrightarrow} \xi_{3},
$$

and under crossing symmetry $\left(\boldsymbol{k} \rightarrow \boldsymbol{k}^{\prime}, \boldsymbol{e} \rightarrow \boldsymbol{e}^{*}\right)$ as

$$
\xi_{1} \stackrel{\text { cross }}{\longrightarrow} \xi_{1}^{\prime}, \quad \xi_{2} \stackrel{\text { cross }}{\longrightarrow}-\xi_{2}^{\prime}, \quad \xi_{3} \stackrel{\text { cross }}{\longrightarrow} \xi_{3}^{\prime} .
$$

\subsubsection{Nucleon}

The nucleon polarization density matrix $\rho_{N}$ is characterized by the 4-polarization vector $S=\left(S_{0}, \boldsymbol{\varsigma}\right)$ which is orthogonal to the nucleon momentum $p=(E, \boldsymbol{p})$ [84]:

$$
\begin{aligned}
\rho_{N} & \equiv u_{\lambda_{N}}(p) \bar{u}_{\lambda_{N}}(p), \\
& =\frac{1}{2}(\gamma p+m)\left(1+\gamma_{5} \gamma S\right),
\end{aligned}
$$


where

$$
\gamma^{0}=\left(\begin{array}{cc}
1 & 0 \\
0 & -1
\end{array}\right), \quad \gamma=\left(\begin{array}{rr}
0 & \boldsymbol{\sigma} \\
-\boldsymbol{\sigma} & 0
\end{array}\right), \quad \gamma^{5}=\left(\begin{array}{rr}
-1 & 0 \\
0 & 1
\end{array}\right)
$$

$u_{\lambda_{N}}(p)$ in Eq. (4.11) is the Dirac's spinor that satisfies

$$
(\gamma p-m) u_{\lambda_{N}}(p)=0
$$

In the nucleon rest frame, the density matrix of Eq. (4.11) is reduced to

$$
\begin{aligned}
\rho_{N} & =\frac{1}{2} m\left(\gamma^{0}+1\right)\left(1-\gamma^{5} \boldsymbol{\gamma} \cdot \boldsymbol{\varsigma}\right), \\
& =\frac{1}{2}(\mathbf{1}+\boldsymbol{\sigma} \cdot \boldsymbol{\varsigma}) .
\end{aligned}
$$

$\boldsymbol{\varsigma}$ is a 3-polarization vector that is related to $S$ by boots transformation as follows:

$$
\boldsymbol{S}=\boldsymbol{\varsigma}+\frac{S_{0}}{E+m} \boldsymbol{p}, \quad S_{0}=\frac{\boldsymbol{p} \cdot \boldsymbol{S}}{E}=\frac{\boldsymbol{p} \cdot \boldsymbol{\varsigma}}{m} .
$$

In a pure state, $|\boldsymbol{\varsigma}|=1$ and in a mixed state, $|\boldsymbol{\varsigma}| \leq 1$.

\subsection{Polarization observables}

\subsubsection{General forms}

A differential cross section $d \sigma / d \Omega$, defined as a square of a scattering amplitude, can be obtained from a density matrix of a final system $\rho_{f}$ :

$$
\begin{aligned}
\frac{d \sigma}{d \Omega} & \equiv \Gamma^{2}\left|T_{f i}\right|^{2} \\
& =\Gamma^{2} \operatorname{Tr}\left(T^{\dagger} \rho_{i} T\right), \\
& =\Gamma^{2} \operatorname{Tr} \rho_{f}
\end{aligned}
$$

with

$$
\rho_{f} \equiv T^{\dagger} \rho_{i} T
$$

In the case of a two-particle reaction, an initial polarization matrix $\rho_{i}$ is given by a direct product of two tensors:

$$
\rho_{i}=\rho_{l} \otimes \rho_{m},
$$

where $\operatorname{Tr} \rho_{i}=1$. Thus, the initial density matrix of the process $\gamma N \rightarrow \gamma N$ is reduced to the tensor product of the $\rho_{\gamma}$ and $\rho_{N}$. Using the expressions in Eqs. (4.3) and (4.14) 
the following initial density matrix is found:

$$
\begin{aligned}
\rho_{i} & =\rho_{\gamma} \otimes \rho_{N}, \\
& =\frac{1}{4}\left(\mathbf{1}+\sum_{j=1}^{3} \xi_{j} \sigma_{j}\right)\left(\mathbf{1}+\sum_{k=x}^{z} \varsigma_{k} \sigma_{k}\right), \\
& =\frac{1}{4}\left(\mathbf{1}+\sum_{j=1}^{3} \xi_{j} \sigma_{j} \mathbf{1}+\sum_{k=x}^{z} \varsigma_{k} \mathbf{1} \sigma_{k}+\sum_{j=1}^{3} \sum_{k=x}^{z} \xi_{j} \varsigma_{k} \sigma_{j k}\right) .
\end{aligned}
$$

The spin matrices $\sigma_{j k}=\sigma_{j} \otimes \sigma_{k}$, appearing in the calculation of Compton polarization observables, are summerized in Appendix F. From Eqs. (4.16) and (4.19) it follows that

$$
\begin{aligned}
\frac{d \sigma}{d \Omega} & =\frac{1}{4} \Gamma^{2} \operatorname{Tr}\left(T^{\dagger}\left\{\mathbf{1}+\sum_{j=1}^{3} \xi_{j} \sigma_{j} \mathbf{1}+\sum_{k=x}^{z} \varsigma_{k} \mathbf{1} \sigma_{k}+\sum_{j=1}^{3} \sum_{k=x}^{z} \xi_{j} \varsigma_{k} \sigma_{j k}\right\} T\right), \\
& =\frac{1}{4} \Gamma^{2} \operatorname{Tr}\left(T^{\dagger} T\right)\left\{\mathbf{1}+\sum_{j=1}^{3} \xi_{j} \Sigma_{j}+\sum_{k=x}^{z} \varsigma_{k} \Sigma_{k}+\sum_{j=1}^{3} \sum_{k=x}^{z} \xi_{j} \varsigma_{k} \Sigma_{j k}\right\} \\
& =\frac{d \bar{\sigma}}{d \Omega}\left(\mathbf{1}+\sum_{j=1}^{3} \xi_{j} \Sigma_{j}+\sum_{k=x}^{z} \varsigma_{k} \Sigma_{k}+\sum_{j=1}^{3} \sum_{k=x}^{z} \xi_{j} \varsigma_{k} \Sigma_{j k}\right),
\end{aligned}
$$

where the photon $\Sigma_{j}$, the nucleon $\Sigma_{k}$ and the photon-nucleon $\Sigma_{j k}$ asymmetries are defined as

$$
\Sigma_{j} \equiv \frac{\operatorname{Tr}\left(T^{\dagger} \sigma_{j} \mathbf{1} T\right)}{\operatorname{Tr}\left(T^{\dagger} T\right)}, \quad \Sigma_{k} \equiv \frac{\operatorname{Tr}\left(T^{\dagger} \mathbf{1} \sigma_{k} T\right)}{\operatorname{Tr}\left(T^{\dagger} T\right)}, \quad \Sigma_{j k} \equiv \frac{\operatorname{Tr}\left(T^{\dagger} \sigma_{j k} T\right)}{\operatorname{Tr}\left(T^{\dagger} T\right)},
$$

respectively. In the unpolarized case $\left(\rho_{\gamma}=\rho_{N}=1 / 2\right)$ the unpolarized differential cross section $d \bar{\sigma} / d \Omega$ results in

$$
\frac{d \bar{\sigma}}{d \Omega}=\frac{1}{4} \Gamma^{2} \operatorname{Tr}\left(T^{\dagger} T\right)
$$

Hereafter, we will represent the observables omitting the phase factor $\Gamma^{2}$.

For polarized Compton scattering, in which either a photon or a nucleon or both of a photon and a nucleon in an initial state are polarized, there are only eight sets of the independent observables [15], which are listed in Table 4.1. As shown in this table, there are two single polarization observables. One is the asymmetry $\Sigma_{3}$ for the photon linearly polarized either parallel ( $x$ axis) or perpendicular ( $y$ axis) to scattering plane (here $x z$ plane). The other is the nucleon asymmetry $\Sigma_{y}$ along the $\pm y$ directions. They are expressed by means of cross sections as

$$
\Sigma_{3}=\left(\frac{d \sigma^{\|}-d \sigma^{\perp}}{d \sigma^{\|}+d \sigma^{\perp}}\right)_{\varsigma=0}, \quad \Sigma_{y}=\left(\frac{d \sigma_{+y}-d \sigma_{-y}}{d \sigma_{+y}+d \sigma_{-y}}\right)_{\xi=0} .
$$


Table 4.1.: The set of the independent observables of nucleon Compton scattering. The quantities $x, y$ and $z$ denote the directions of the nucleon polarization. For the photon, 2 refers to the circular polarization, and 3 stands for the linear polarization parallel or perpendicular to the scattering plane. The number 1 means the linear polarization of the photon with the angle $\varphi=\pi / 4$ with respect to the scattering plane.

\begin{tabular}{|c|c|c|c|c|}
\hline \multirow{2}{*}{$\begin{array}{c}\text { photon } \\
\text { nucleon }\end{array}$} & unpolarization & \multicolumn{2}{|c|}{ linear polarization } & $\begin{array}{c}\text { circular polarization } \\
\xi_{2}= \pm 1\end{array}$ \\
\cline { 3 - 5 } unpolarization & $\frac{d \bar{\sigma}}{d \Omega}$ & $\Sigma_{3}= \pm 1$ & $\xi_{1}= \pm 1$ & \\
\hline$\varsigma_{1=x}$ & & & $\Sigma_{1 x}$ & $\Sigma_{2 x}$ \\
\hline$\varsigma_{2=y}$ & $\Sigma_{y}$ & $\Sigma_{3 y}$ & & \\
\hline$\varsigma_{3=z}$ & & & $\Sigma_{1 z}$ & $\Sigma_{2 z}$ \\
\hline
\end{tabular}

In the case of double polarized Compton scattering there are in total five independent measurable quantities. For the incoming photon linearly polarized with respect to the $x(y)$ axis and the target-nucleon polarized in the direction of $\pm y$ axis, the beam-target asymmetry $\Sigma_{3 y}$ is represented as

$$
\Sigma_{3 y}=\frac{\left(d \sigma^{\|}-d \sigma^{\perp}\right)_{+y}-\left(d \sigma^{\|}-d \sigma^{\perp}\right)_{-y}}{\left(d \sigma^{\|}+d \sigma^{\perp}\right)_{+y}+\left(d \sigma^{\|}+d \sigma^{\perp}\right)_{-y}}
$$

If the photon is linearly polarized at $\varphi= \pm \pi / 4$ with respect to the scattering plane, and the nucleon is polarized along the $x$ or $z$ direction, respectively, there are then two measurable asymmetries $\Sigma_{1 x}$ and $\Sigma_{1 z}$ given by

$$
\Sigma_{1 x}=\frac{d \sigma_{x}^{\pi / 4}-d \sigma_{x}^{-\pi / 4}}{d \sigma_{x}^{\pi / 4}+d \sigma_{x}^{-\pi / 4}}, \quad \Sigma_{1 z}=\frac{d \sigma_{z}^{\pi / 4}-d \sigma_{z}^{-\pi / 4}}{d \sigma_{z}^{\pi / 4}+d \sigma_{z}^{-\pi / 4}}
$$

For the circularly polarized photon off the nucleon polarized in $x$ or $z$ direction, the asymmetries $\Sigma_{2 x}$ and $\Sigma_{2 z}$, formulated as

$$
\Sigma_{2 x}=\frac{d \sigma_{x}^{R}-d \sigma_{x}^{L}}{d \sigma_{x}^{R}+d \sigma_{x}^{L}}, \quad \Sigma_{2 z}=\frac{d \sigma_{z}^{R}-d \sigma_{z}^{L}}{d \sigma_{z}^{R}+d \sigma_{z}^{L}},
$$

can be measured. By virtue of the relations,

$$
d \sigma_{i}^{R}=d \sigma_{-i}^{L}, \quad d \sigma_{i}^{\varphi}=d \sigma_{-i}^{-\varphi}, \quad(i= \pm x, \pm z)
$$


the asymmetries of Eqs. (4.25) and (4.26) have the opposite signs under the transformations of $x \rightarrow-x$ and $z \rightarrow-z$.

By introducing the quantities [15] of

$$
\begin{aligned}
& F_{1}=\frac{d \sigma^{\pi / 4}-d \sigma^{-\pi / 4}}{2 d \bar{\sigma}}=\Sigma_{1 x} \varsigma_{x}+\Sigma_{1 z} \varsigma_{z}, \\
& F_{2}=\frac{d \sigma^{R}-d \sigma^{L}}{2 d \bar{\sigma}}=\Sigma_{2 x} \varsigma_{x}+\Sigma_{2 z} \varsigma_{z}, \\
& F_{3}=\frac{d \sigma^{\|}-d \sigma^{\perp}}{2 d \bar{\sigma}}=\Sigma_{3}+\Sigma_{3 y} \varsigma_{y},
\end{aligned}
$$

the differential cross section of Eq. (4.20) can then be written in a compact form of

$$
\frac{d \sigma}{d \Omega}=\frac{d \bar{\sigma}}{d \Omega}\left\{1+\Sigma_{y} \varsigma_{y}+\boldsymbol{F} \cdot \boldsymbol{\xi}\right\},
$$

which leads to the following differential cross sections in the case of each polarization mentioned above

$$
\begin{aligned}
\frac{d \sigma^{\|(\perp)}}{d \Omega} & =\frac{d \bar{\sigma}}{d \Omega}\left\{1 \pm \Sigma_{3}\right\}, \\
\frac{d \sigma_{y}(-y)}{d \Omega} & =\frac{d \bar{\sigma}}{d \Omega}\left\{1 \pm \Sigma_{y}\right\}, \\
\frac{d \sigma_{y}^{\|}(-y)}{d \Omega} & =\frac{d \bar{\sigma}}{d \Omega}\left\{1 \pm \Sigma_{y}+\Sigma_{3} \pm \Sigma_{3 y}\right\}, \\
\frac{d \sigma_{y}^{\perp}(-y)}{d \Omega} & =\frac{d \bar{\sigma}}{d \Omega}\left\{1 \pm \Sigma_{y}-\Sigma_{3} \mp \Sigma_{3 y}\right\}, \\
\frac{d \sigma_{x}^{\pi / 4(-\pi / 4)}}{d \Omega} & =\frac{d \bar{\sigma}}{d \Omega}\left\{1 \pm \Sigma_{1 x}\right\}, \\
\frac{d \sigma_{z}^{\pi / 4(-\pi / 4)}}{d \Omega} & =\frac{d \bar{\sigma}}{d \Omega}\left\{1 \pm \Sigma_{1 z}\right\}, \\
\frac{d \sigma_{x}^{R(L)}}{d \Omega} & =\frac{d \bar{\sigma}}{d \Omega}\left\{1 \pm \Sigma_{2 x}\right\}, \\
\frac{d \sigma_{z}^{R(L)}}{d \Omega} & =\frac{d \bar{\sigma}}{d \Omega}\left\{1 \pm \Sigma_{2 z}\right\} .
\end{aligned}
$$

\subsubsection{Formalismus in helicity and invariant amplitudes}

With the help of the $T$-matrix of Eq. (2.13) represented by the helicity amplitudes or of the relation between the amplitudes $H_{i}$ and $A_{i}$ of Eq. (D.1), one can get the observables in terms of the helicity $H_{i}$ or the invariant $A_{i}$ amplitudes. 
The c.m. unpolarized differential cross section $d \bar{\sigma} / d \Omega^{*}$ reads then

$$
\begin{aligned}
\frac{d \bar{\sigma}}{d \Omega^{*}}= & \frac{1}{2}\left\{\left|H_{1}\right|^{2}+\left|H_{2}\right|^{2}+2\left|H_{3}\right|^{2}+2\left|H_{4}\right|^{2}+\left|H_{5}\right|^{2}+\left|H_{6}\right|^{2}\right\}, \\
= & \frac{1}{4}\left\{\left(4 m^{2}-t\right)\left(t^{2}\left|A_{1}\right|^{2}+\eta^{2}\left|A_{3}\right|^{2}\right)-t^{3}\left|A_{2}\right|^{2}+\eta^{3}\left|A_{4}\right|^{2}\right. \\
& -4 \nu^{2} t\left(t+8 \nu^{2}\right)\left|A_{5}\right|^{2}+2 \eta\left(t^{2}+2 m^{2} \eta\right)\left|A_{6}\right|^{2} \\
& \left.+4 \operatorname{Re}\left[2 \nu^{2} t^{2}\left(A_{1}+A_{2}\right) A_{5}^{*}+\frac{1}{2} \eta^{2}\left(4 m^{2} A_{3}+t A_{4}\right) A_{6}^{*}\right]\right\} .
\end{aligned}
$$

As shown in Eq. (4.38) an unpolarized differential cross section is simply given as the sum of a squares of the individual helicity amplitude $\left|H_{i}\right|^{2}$, and is then averaged over the four possible initial states. The $\left|H_{i}\right|^{2}$ is not measurable directly because the polarizations in a final state are determined by the interaction between the particles. Thus, it is allowed to measure only the different linear combinations of them, i.e differential cross sections or asymmetries. The explicite expressions of the single and double polarization observables are as follows:

$$
\begin{aligned}
& \frac{d \bar{\sigma}}{d \Omega^{*}} \Sigma_{3}=\frac{1}{4} \operatorname{Tr}\left(T^{\dagger} \sigma_{x} \mathbf{1} T\right), \\
&=-\operatorname{Re}\left\{\left(H_{1}+H_{2}\right) H_{4}^{*}+\left(H_{5}+H_{6}\right) H_{3}^{*}\right\}, \\
&=\frac{\eta t}{2} \operatorname{Re}\left\{\left(\left(4 m^{2}-t\right) A_{1}+4 \nu^{2} A_{5}\right) A_{3}^{*}+4 m^{2} A_{1} A_{6}^{*}\right\}, \\
& \frac{d \bar{\sigma}}{d \Omega^{*}} \Sigma_{y}=\frac{1}{4} \operatorname{Tr}\left(T^{\dagger} \mathbf{1} \sigma_{y} T\right), \\
&=\operatorname{Im}\left\{\left(H_{1}+H_{2}\right) H_{3}^{*}-\left(H_{5}+H_{6}\right) H_{4}^{*}\right\}, \\
&=-2 m \nu \sqrt{-\eta t} \operatorname{Im}\left\{t A_{1} A_{5}^{*}+\eta A_{3} A_{6}^{*}\right\}, \\
& \frac{d \bar{\sigma}}{d \Omega^{*}} \Sigma_{3 y}=\frac{1}{4} \operatorname{Tr}\left(T^{\dagger} \sigma_{x y} T\right), \\
&=-\operatorname{Im}\left\{H_{1} H_{5}^{*}+H_{2} H_{6}^{*}-2 H_{3} H_{4}^{*}\right\}, \\
&=\frac{m}{4} \sqrt{-\eta t} \operatorname{Im}\left\{-8 \nu\left[\left(t A_{1}-\left(t+4 \nu^{2}\right) A_{5}\right) A_{6}^{*}+\eta A_{3} A_{5}^{*}\right]\right. \\
&\left.\quad+\frac{2}{m}\left(t A_{2}-4 \nu^{2} A_{5}\right)\left(\eta A_{4}^{*}+t A_{6}^{*}\right)\right\},
\end{aligned}
$$




$$
\begin{aligned}
& \frac{d \bar{\sigma}}{d \Omega^{*}} \Sigma_{1 x}=\frac{1}{4} \operatorname{Tr}\left(T^{\dagger} \sigma_{y x} T\right), \\
& =\operatorname{Im}\left\{H_{1} H_{5}^{*}-H_{2} H_{6}^{*}\right\} \text {, } \\
& =\frac{\sqrt{-\eta t}}{4\left(s-m^{2}\right)} \operatorname{Im}\left\{4 m\left(t A_{2}-4 \nu^{2} A_{5}\right)\left(m \eta A_{3}^{*}-\left(\nu t-m\left(t+4 \nu^{2}\right)\right) A_{6}^{*}\right)\right. \\
& +\left(t(t-4 m(m+\nu)) A_{1}+4 \nu\left(m\left(t+4 \nu^{2}\right)-\nu t\right) A_{5}\right) \\
& \left.\left(\eta A_{4}^{*}+t A_{6}^{*}\right)\right\} \\
& \frac{d \bar{\sigma}}{d \Omega^{*}} \Sigma_{1 z}=\frac{1}{4} \operatorname{Tr}\left(T^{\dagger} \sigma_{y z} T\right), \\
& =\operatorname{Im}\left\{\left(H_{1}-H_{2}\right) H_{4}^{*}+\left(H_{5}-H_{6}\right) H_{3}^{*}\right\}, \\
& =-\frac{1}{8 m^{2}\left(s-m^{2}\right)} \operatorname{Im}\left\{4 m t ( t A _ { 2 } - 4 \nu ^ { 2 } A _ { 5 } ) \left[-m \eta\left(m^{2}+s\right) A_{3}^{*}\right.\right. \\
& \left.+\left(2 \nu\left(m^{2}-s\right)^{2}+\left(m^{2}+s\right)\left(\nu t-m\left(t+4 \nu^{2}\right)\right)\right) A_{6}^{*}\right] \\
& +\left[4 m t^{2}\left(2\left(m^{2}-s\right)^{2}+t\left(m^{2}+s\right)\right) A_{1}\right. \\
& -t\left(2\left(m^{2}-s\right)^{2}+\left(m^{2}+s\right)(t-2 \nu)\right)\left(t A_{1}-4 \nu^{2} A_{5}\right) \\
& \left.\left.-2 \nu t^{2}\left(m^{2}+s\right) A_{5}\right]\left(\eta A_{4}^{*}+t 4 \nu^{2} A_{5}^{*}\right)\right\} \text {, } \\
& \frac{d \bar{\sigma}}{d \Omega^{*}} \Sigma_{2 x}=\frac{1}{4} \operatorname{Tr}\left(T^{\dagger} \sigma_{z x} T\right), \\
& =\operatorname{Re}\left\{\left(H_{5}-H_{6}\right) H_{4}^{*}-\left(H_{1}-H_{2}\right) H_{3}^{*}\right\}, \\
& =\frac{\sqrt{-\eta t}}{4\left(s-m^{2}\right)} \operatorname{Re}\left\{4 m^{2} t^{2} A_{2} A_{1}^{*}-\eta(t-4 m(m+\nu)) A_{3}\left(\eta A_{4}^{*}+t A_{6}^{*}\right)\right. \\
& \left.-16 m t \nu^{2} A_{5}\left(m A_{1}^{*}-\nu A_{5}^{*}\right)\right\} \\
& \frac{d \bar{\sigma}}{d \Omega^{*}} \Sigma_{2 z}=\frac{1}{4} \operatorname{Tr}\left(T^{\dagger} \sigma_{z z} T\right), \\
& =\frac{1}{2}\left\{\left|H_{2}\right|^{2}+\left|H_{6}\right|^{2}-\left|H_{1}\right|^{2}-\left|H_{5}\right|^{2}\right\}, \\
& =\frac{1}{8 m^{2}\left(s-m^{2}\right)}\left\{4 m t \left[m t\left(m^{2}+s\right)\left(t A_{2}-4 \nu^{2} A_{5}\right) A_{1}^{*}\right.\right. \\
& \left.+4 \nu^{3}\left(2\left(m^{2}-s\right)^{2}+\left(m^{2}+s\right) t\right) A_{5} A_{5}^{*}\right] \\
& +\eta\left[\left(2\left(m^{2}-s\right)^{2}+\left(m^{2}+s\right) t\right)\left(\left(4 m^{2}-t\right) A_{3}+4 m^{2} A_{6}\right)\right. \\
& \left.\left.+4 m\left(m^{2}+s\right) \nu t\right]\left(\eta A_{4}^{*}+t A_{6}^{*}\right)\right\} \text {. }
\end{aligned}
$$


As said, $H_{3}=H_{4}=H_{5}=H_{6}=0$ at $\theta=0(t=0)$ and $H_{1}=H_{2}=H_{3}=H_{4}=0$ at $\theta=\pi(\eta=0)$. In Eqs. (4.38)-(4.53), it is hence simply recognizable that all observables, excepting the c.m. unpolarized differential cross section, $\Sigma_{1 z}$ and $\Sigma_{2 z}$, are equal to zero at these two extreme angles. Notice also that below the pion threshold $(\omega=150 \mathrm{MeV})$ there are only three asymmetries, $\Sigma_{3}, \Sigma_{2 x}$, and $\Sigma_{2 z}$, which are different from zero. Indeed, $\Sigma_{2 z}$ is the only measurable quantity in this region of energy and at the angles $\theta=0$ and $\pi$. From

$$
\begin{aligned}
& {\left[\frac{d \bar{\sigma}}{d \Omega^{*}}\right]_{\theta=0}=\frac{1}{2}\left\{\left|H_{1}\right|^{2}+\left|H_{2}\right|^{2}\right\},} \\
& {\left[\frac{d \bar{\sigma}}{d \Omega^{*}} \Sigma_{2 z}\right]_{\theta=0}=\frac{1}{2}\left\{\left|H_{2}\right|^{2}-\left|H_{1}\right|^{2}\right\},}
\end{aligned}
$$

and

$$
\begin{aligned}
& {\left[\frac{d \bar{\sigma}}{d \Omega^{*}}\right]_{\theta=\pi}=\frac{1}{2}\left\{\left|H_{5}\right|^{2}+\left|H_{6}\right|^{2}\right\},} \\
& {\left[\frac{d \bar{\sigma}}{d \Omega^{*}} \Sigma_{2 z}\right]_{\theta=\pi}=\frac{1}{2}\left\{\left|H_{6}\right|^{2}-\left|H_{5}\right|^{2}\right\} .}
\end{aligned}
$$

followed

$$
\begin{aligned}
\left|H_{1}\right|^{2} & =\left[\frac{d \bar{\sigma}}{d \Omega^{*}}\left(1-\Sigma_{2 z}\right)\right]_{\theta=0}=\left[\frac{d \sigma_{z}^{L}}{d \Omega^{*}}\right]_{\theta=0}, \\
\left|H_{2}\right|^{2} & =\left[\frac{d \bar{\sigma}}{d \Omega^{*}}\left(1+\Sigma_{2 z}\right)\right]_{\theta=0}=\left[\frac{d \sigma_{z}^{R}}{d \Omega^{*}}\right]_{\theta=0}
\end{aligned}
$$

and

$$
\begin{aligned}
\left|H_{5}\right|^{2} & =\left[\frac{d \bar{\sigma}}{d \Omega^{*}}\left(1-\Sigma_{2 z}\right)\right]_{\theta=\pi}=\left[\frac{d \sigma_{z}^{L}}{d \Omega^{*}}\right]_{\theta=\pi}, \\
\left|H_{6}\right|^{2} & =\left[\frac{d \bar{\sigma}}{d \Omega^{*}}\left(1+\Sigma_{2 z}\right)\right]_{\theta=\pi}=\left[\frac{d \sigma_{z}^{R}}{d \Omega^{*}}\right]_{\theta=\pi} .
\end{aligned}
$$

As a result, the magnitudes of the helicity amplitudes $H_{1}, H_{2}, H_{5}$ and $H_{6}$ can therefore be extracted from the polarized cross sections $d \sigma_{z}^{R} / d \Omega^{*}$ and $d \sigma_{z}^{L} / d \Omega^{*}$ at these two angles.

On the other hand, the cross section $d \bar{\sigma} / d \Omega^{*}$ and the asymmetry $\Sigma_{2 z}$ are also determined by the amplitudes $A_{3,4}$ and $A_{6}$ at the angle $\theta=0$ and by the amplitudes $A_{1,2}$ and $A_{5}$ at the angle $\theta=\pi$, respectively:

$$
\begin{aligned}
& {\left[\frac{d \bar{\sigma}}{d \Omega^{*}}\right]_{\theta=0}=m^{2} \eta^{2}\left(\left|A_{3}\right|^{2}+\left|A_{6}\right|^{2}\right)+\frac{1}{4} \eta^{3}\left|A_{4}\right|^{2}+2 m^{2} \eta^{2} \operatorname{Re}\left[A_{3} A_{6}^{*}\right],} \\
& {\left[\frac{d \bar{\sigma}}{d \Omega^{*}} \Sigma_{2 z}\right]_{\theta=0}=\eta^{2}\left(m^{2}-s\right)\left(A_{3}+A_{6}\right) A_{4}^{*} .}
\end{aligned}
$$


And

$$
\begin{aligned}
{\left[\frac{d \bar{\sigma}}{d \Omega^{*}}\right]_{\theta=\pi}=\frac{1}{4}\left\{\left(4 m^{2}-t\right) t^{2}\left|A_{1}\right|^{2}-t^{3}\left|A_{2}\right|^{2}-4 \nu^{2} t\left(t+8 \nu^{2}\right)\left|A_{5}\right|^{2}\right.} \\
\left.+8 \nu^{2} t^{2} \operatorname{Re}\left[\left(A_{1}+A_{2}\right) A_{5}^{*}\right]\right\} \\
{\left[\frac{d \bar{\sigma}}{d \Omega^{*}} \Sigma_{2 z}\right]_{\theta=\pi}=\frac{t}{2 m\left(s-m^{2}\right)}\left\{m t\left(m^{2}+s\right)\left(t A_{2}-4 \nu^{2} A_{5}\right) A_{1}^{*}\right.} \\
\left.+4 \nu^{3}\left(2\left(m^{2}-s\right)^{2}+\left(m^{2}+s\right) t\right) A_{5} A_{5}^{*}\right\}
\end{aligned}
$$

In order to investigate the sensitivity of the observables to the invariant amplitudes, we turn the strength of the each invariant amplitude $A_{i}$ from $100 \%$ to $101 \%$ and illustrate the difference between the results by using the invariant amplitude with the unchanged strength and that obtained from the amplitudes $A_{i}$ having increased strength in Fig. 4.1-4.6 by means of the solid $\left(\theta=30^{\circ}\right)$, dashed $\left(\theta=60^{\circ}\right)$, dotted $\left(\theta=120^{\circ}\right)$ and dashed-dotted lines $\left(\theta=150^{\circ}\right)$, respectively.

At the small angles $\left(\theta=30^{\circ}\right.$ and $\left.60^{\circ}\right)$ the maximum respone to a change of $A_{3,4}$ and $A_{6}$ is, as expected, shown in the asymmetry $\Sigma_{2 z}$. Furthermore, the effects of the amplitudes $A_{3}$ and $A_{6}$ on $\Sigma_{2 z}$ are most visible just above and below the pion threshold, i.e. $\omega \simeq 130 \mathrm{MeV}$ as well as about $\omega \simeq 170 \mathrm{MeV}$. Since the sum of the $A_{3}$ and $A_{6}$ is connected with the $\alpha+\beta$ at the angle $\theta=0$, the measurment of the asymmetry $\Sigma_{2 z}$ in this kinematical region could offer important information on the electromagnetic polarizability. On the other hand, the influence of changing the $A_{4}$ amplitude is seen not only for $\Sigma_{2 z}$ at higher energies, but also for $\Sigma_{1 x}$ at the large angle $\theta=120^{\circ}$ and above the energy of the second resonance $(\omega \simeq 750 \mathrm{MeV})$. The sensitivity of the amplitudes $A_{3}$ and $A_{6}$ are generally observed in the low energy region and at the small angles in nearly all asymmetries. In the case of the unpolarized differential cross section, the dependence on the amplitudes $A_{3,4}$ and $A_{6}$ is specifically large at $\omega \simeq 320 \mathrm{MeV}$ and around the energies $\omega \simeq 750 \mathrm{MeV}$, respectively.

At the large backward angles of $\theta=120^{\circ}$ and $150^{\circ}$ the change of the strength of the amplitudes $A_{2}$ and $A_{5}$ has the largest influence on $\Sigma_{2 z}$ at the energy $\omega \simeq 730 \mathrm{MeV}$. This means that the measurment of the asymmetry $\Sigma_{2 z}$ in this high energy region and at the larger angles is appropriate to get more knowledge on the proton backward spin polarizability $\gamma_{\pi}^{p}$. In the case of the amplitude $A_{1}$, the asymmetry $\Sigma_{2 z}$ as well as $\Sigma_{3}$ are sensitive to a change of the strength of this amplitude, in particular below the one-pion threshold and in the region of $\Delta$-resonance, respectively. Among the three amplitudes $A_{1}, A_{2}$ and $A_{5}$, the amplitude $A_{1}$ affects mostly the unpolarizaed differential cross sections $d \bar{\sigma} / d \Omega^{*}$.

As a result, the experiment using the circularly polarized photon and the proton-target polarized in $z$ direction is mostly adequate to determine the value of the polarizabilities. 

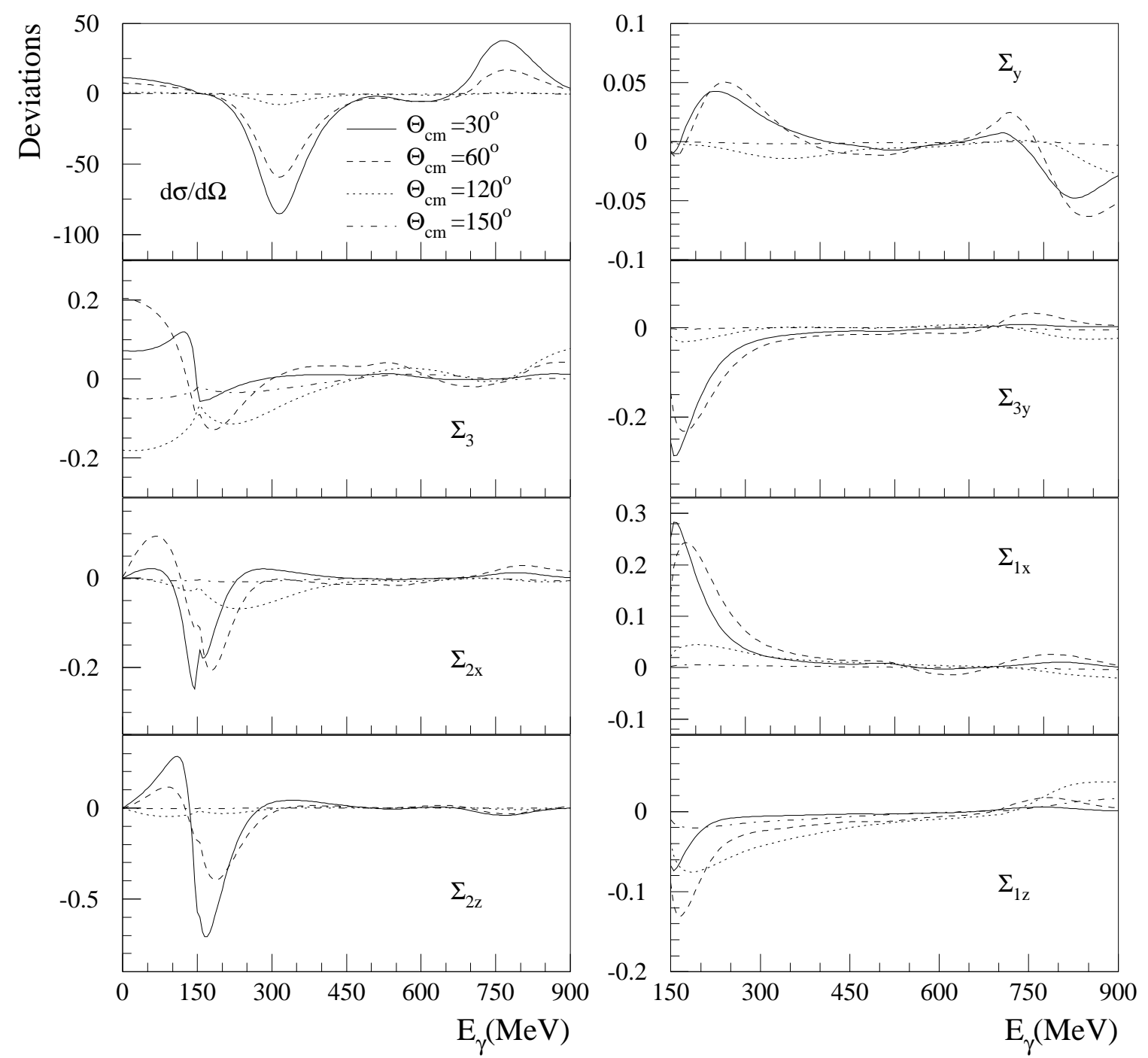

Figure 4.1.: The relative difference between the results for the observables of proton Compton scattering obtained from the invariant amplitude $A_{3}$ with the unchanged strength and that obtained from the amplitudes $A_{3}$ with the strength increased by $1 \%$ at four different angles $\theta_{c m}=30^{\circ}$ (solid lines), $60^{\circ}$ (dashed lines), $120^{\circ}$ (dotted lines) and $150^{\circ}$ (dashed-dotted lines). 

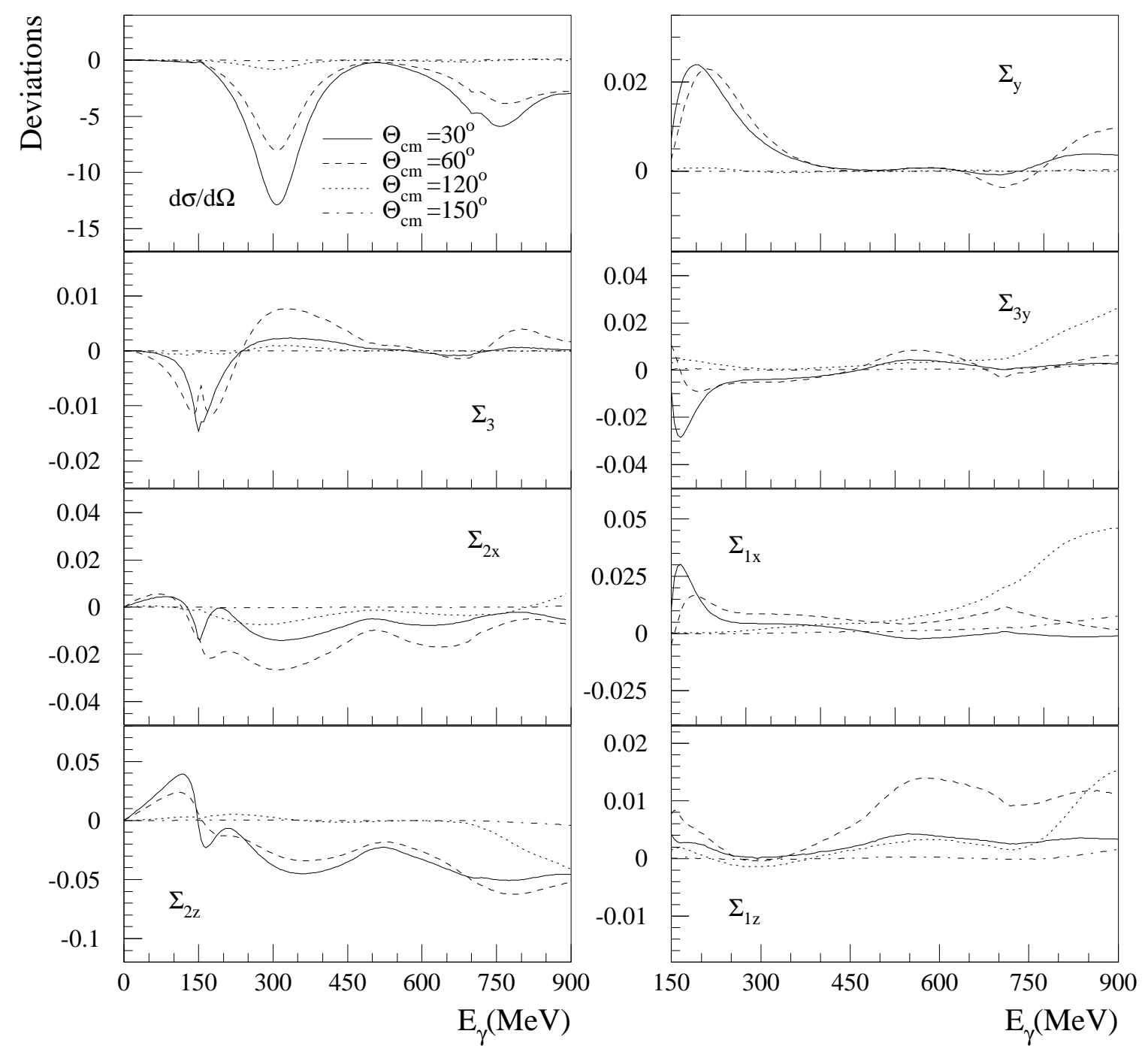

Figure 4.2.: The relative difference between the results for the observables of proton Compton scattering obtained from the invariant amplitude $A_{4}$ with the unchanged strength and that obtained from the amplitudes $A_{4}$ with the strength increased by $1 \%$ at four different angles $\theta_{\mathrm{cm}}=$ $30^{\circ}, 60^{\circ} 120^{\circ}$ and $150^{\circ}$. The notations are the same as in Fig. 4.1. 

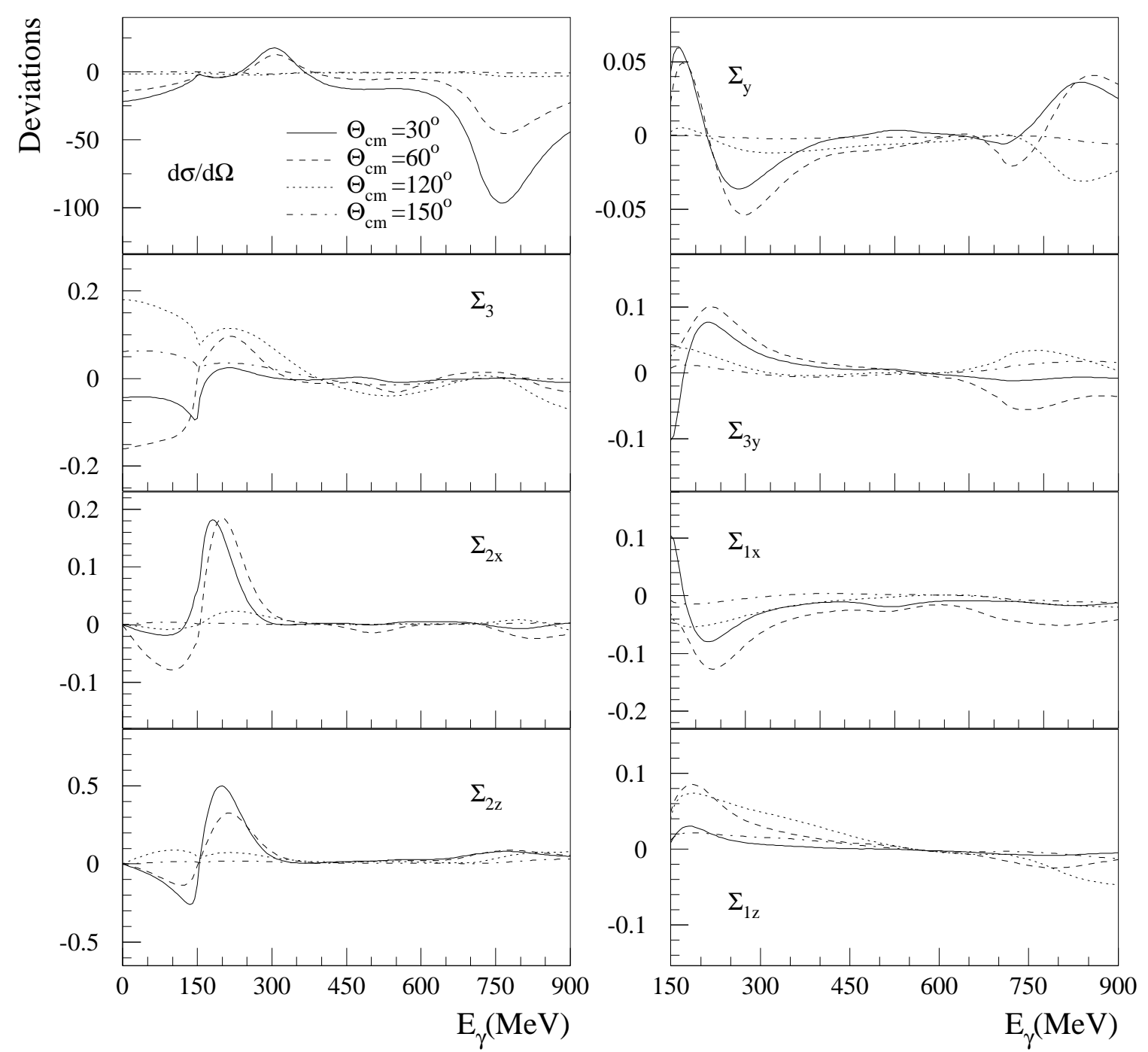

Figure 4.3.: The relative difference between the results for the observables of proton Compton scattering obtained from the invariant amplitude $A_{6}$ with the unchanged strength and that obtained from the amplitudes $A_{6}$ with the strength increased by $1 \%$ at four different angles $\theta_{\mathrm{cm}}=$ $30^{\circ}, 60^{\circ} 120^{\circ}$ and $150^{\circ}$. The notations are the same as in Fig. 4.1. 

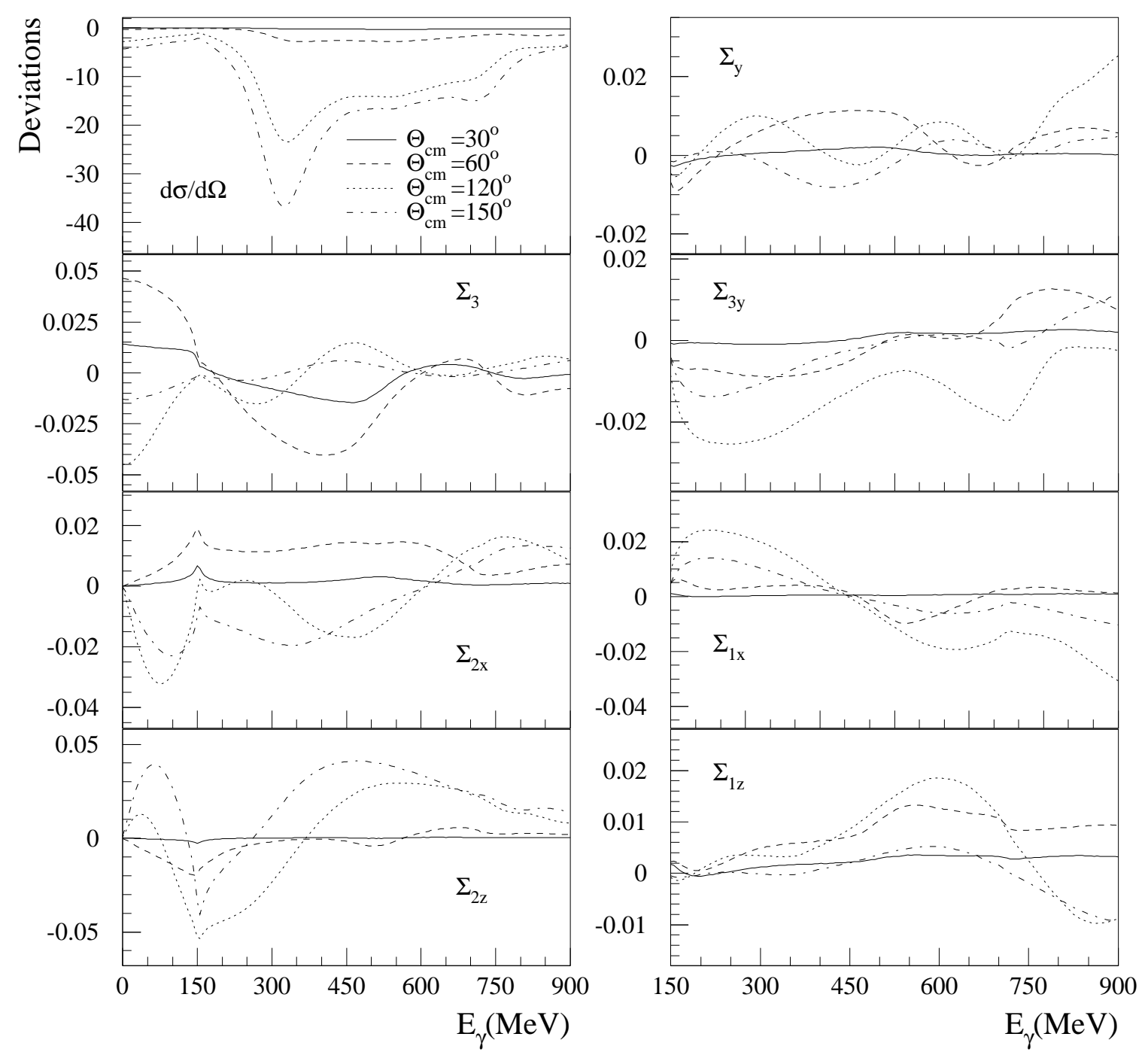

Figure 4.4.: The relative difference between the results for the observables of proton Compton scattering obtained from the invariant amplitude $A_{1}$ with the unchanged strength and that obtained from the amplitudes $A_{1}$ with the strength increased by $1 \%$ at four different angles $\theta_{\mathrm{cm}}=$ $30^{\circ}, 60^{\circ} 120^{\circ}$ and $150^{\circ}$. The notations are the same as in Fig. 4.1. 

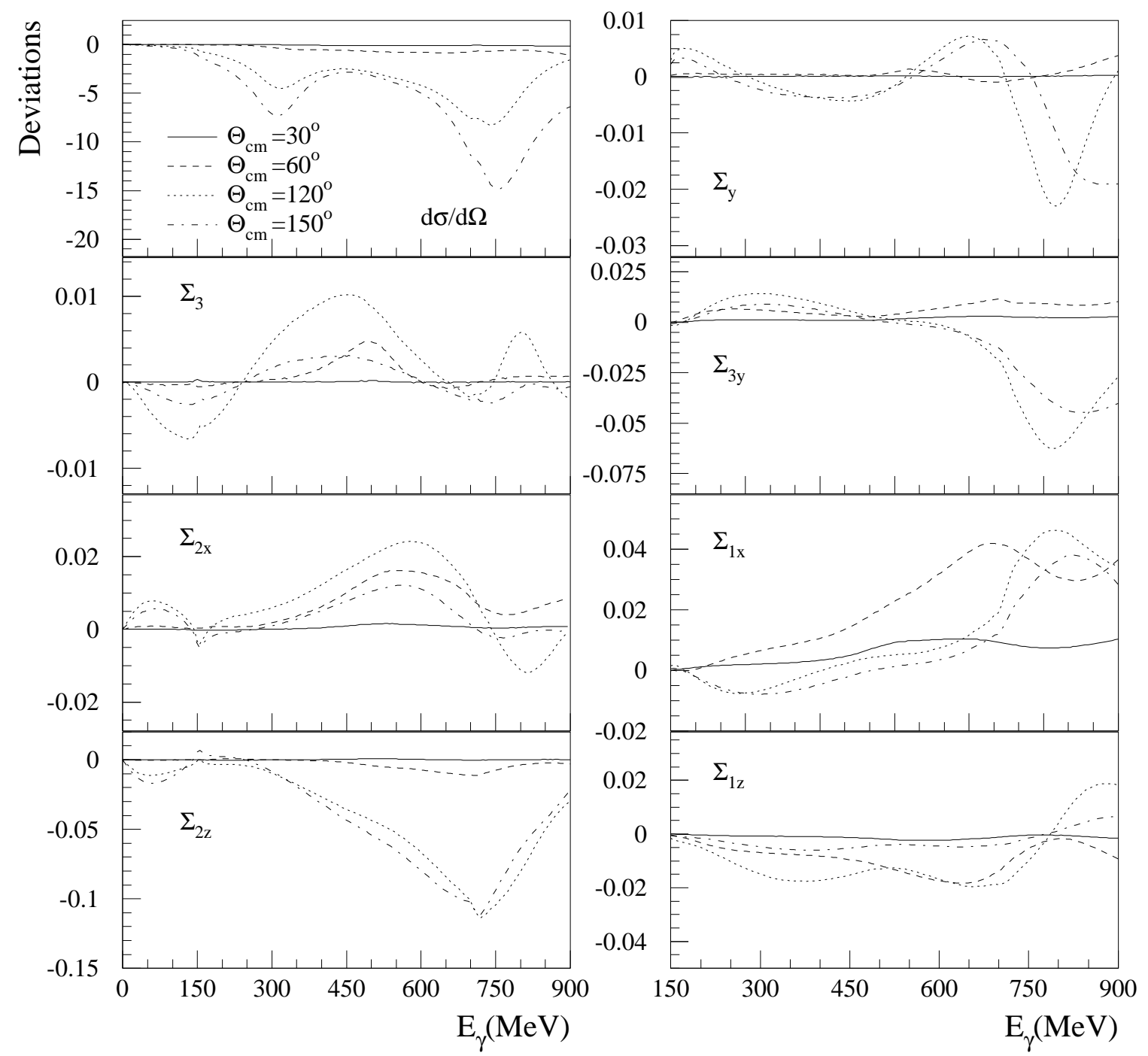

Figure 4.5.: The relative difference between the results for the observables of proton Compton scattering obtained from the invariant amplitude $A_{2}$ with the unchanged strength and that obtained from the amplitudes $A_{2}$ with the strength increased by $1 \%$ at four different angles $\theta_{c m}=$ $30^{\circ}, 60^{\circ} 120^{\circ}$ and $150^{\circ}$. The notations are the same as in Fig. 4.1. 

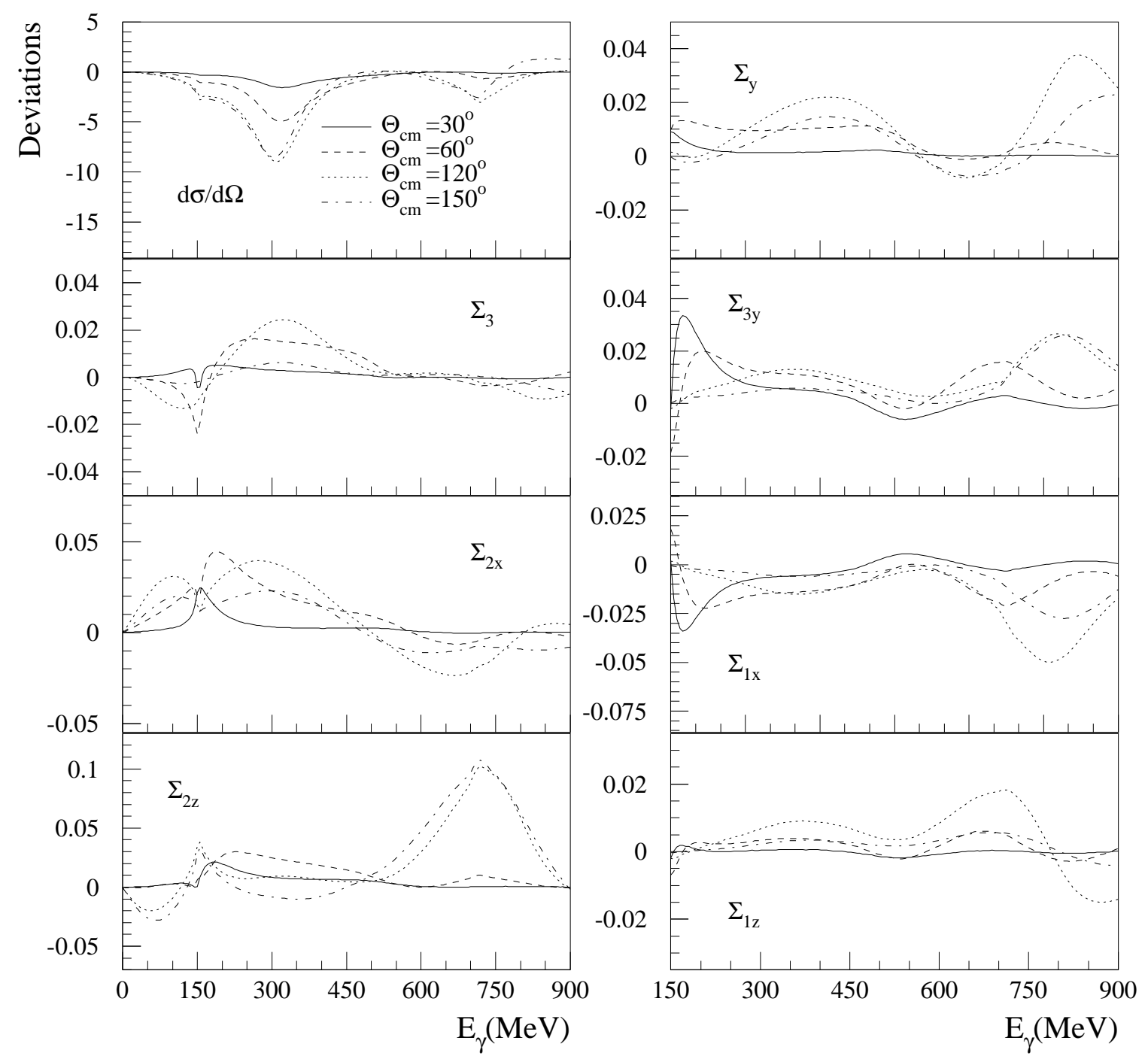

Figure 4.6.: The relative difference between the results for the observables of proton Compton scattering obtained from the invariant amplitude $A_{5}$ with the unchanged strength and that obtained from the amplitudes $A_{5}$ with the strength increased by $1 \%$ at four different angles $\theta_{\mathrm{cm}}=$ $30^{\circ}, 60^{\circ} 120^{\circ}$ and $150^{\circ}$. The notations are the same as in Fig. 4.1. 


\subsubsection{Observables by multipole representation}

The Compton observables can be expressed by the multipole decompositions. Restricting the expansion to $\mathrm{s}$ and $\mathrm{p}$ waves and retaining only terms with the dominant $f_{M M}^{1+}$, one gets the following c.m. unpolarized cross section represented as a power series in $\cos \theta$

$$
\frac{d \bar{\sigma}}{d \Omega^{*}}=a+b \cos \theta+c \cos ^{2} \theta
$$

where

$$
\begin{aligned}
a & =\frac{7}{2}\left|f_{M M}^{1+}\right|^{2}+\operatorname{Re}\left[\left(f_{M M}^{1-}+6 f_{M E}^{1+}\right)\left(f_{M M}^{1+}\right)^{*}\right], \\
b & =2 \operatorname{Re}\left[\left(f_{E E}^{1+}-25 f_{E E}^{1-}+6 f_{E M}^{1+}\right)\left(f_{M M}^{1+}\right)^{*}\right], \\
c & =\frac{3}{2}\left|f_{M M}^{1+}\right|^{2}+3 \operatorname{Re}\left[\left(f_{M M}^{1-}+6 f_{M E}^{1+}\right)\left(f_{M M}^{1+}\right)^{*}\right] .
\end{aligned}
$$

Here the constants $a, b$ and $c$ can be fitted to the experimental data at various energies. It is should be pointed out here that the differential cross section alone determines the magnitude of the wave $f_{M M}^{1+}$ :

$$
\left|f_{M M}^{1+}\right|=\frac{1}{3} \sqrt{3 a-c} .
$$

Less explicitly, $\left|f_{M M}^{1+}\right|$ can also be determined by unpolarized differential cross sections at the angle $\theta=\pi / 2$, if all the interference terms with $f_{M M}^{1+}$ in Eq. (4.63) are ignored:

$$
\left|f_{M M}^{1+}\right|=\sqrt{\frac{2}{7}} a=\sqrt{\left[\frac{d \bar{\sigma}}{d \Omega^{*}}\right]_{\theta=\pi / 2}} .
$$

In the case of the single polarization, the photon $\Sigma_{3}$ and nucleon $\Sigma_{y}$ asymmetries result from Eqs. (4.40) and (4.42)

$$
\frac{d \bar{\sigma}}{d \Omega^{*}} \Sigma_{3}=d \sin ^{2} \theta, \quad \frac{d \bar{\sigma}}{d \Omega^{*}} \Sigma_{y}=\sin \theta(e+f \cos \theta)
$$

with the angle-independent quantities from $d$ to $f$ given by

$$
\begin{aligned}
d & =\frac{3}{2}\left|f_{M M}^{1+}\right|^{2}+3 \operatorname{Re}\left[\left(f_{M M}^{1-}+2 f_{M E}^{1+}\right)\left(f_{M M}^{1+}\right)^{*}\right], \\
e & =3 \operatorname{Im}\left[\left(f_{E E}^{1-}-4 f_{E M}^{1+}\right)\left(f_{M M}^{1+}\right)^{*}\right] \\
f & =-3 \operatorname{Im}\left[\left(f_{M M}^{1-}+4 f_{M E}^{1+}\right)\left(f_{M M}^{1+}\right)^{*}\right] .
\end{aligned}
$$

The photon asymmetry $\Sigma_{3}$ has a clean dependence on $\sin ^{2} \theta$ and can be parametrized by a single energy-dependent parameter $f$. With respect to $\Sigma_{3 y}$

$$
\frac{d \bar{\sigma}}{d \Omega^{*}} \Sigma_{3 y}=\sin \theta(g+h \cos \theta),
$$


with

$$
\begin{aligned}
& g=2 \operatorname{Im}\left[\left(f_{E E}^{1+}-f_{E E}^{1-}-6 f_{E M}^{1+}\right)\left(f_{M M}^{1+}\right)^{*}\right], \\
& h=3 \operatorname{Im}\left[\left(f_{M M}^{1-}-3 f_{E E}^{1-}\right)\left(f_{M M}^{1+}\right)^{*}\right] . \\
& \frac{d \bar{\sigma}}{d \Omega^{*}} \Sigma_{1 x}=\sin \theta\left(i+j \cos \theta+k \cos ^{2} \theta\right), \\
& \frac{d \bar{\sigma}}{d \Omega^{*}} \Sigma_{1 z}=\sin ^{2} \theta(l+m \cos \theta) .
\end{aligned}
$$

and the constants read

$$
\begin{aligned}
i & =-2 \operatorname{Im}\left[\left(f_{E E}^{1+}-f_{E E}^{1-}+f_{M E}^{1+}+3 f_{E M}^{1+}\right)\left(f_{M M}^{1+}\right)^{*}\right] \\
j & =-3 \operatorname{Im}\left[\left(f_{M M}^{1-}-3 f_{E E}^{1-}\right)\left(f_{M M}^{1+}\right)^{*}\right] \\
k & =6 \operatorname{Im}\left[\left(f_{M E}^{1+}+f_{E M}^{1+}\right)\left(f_{M M}^{1+}\right)^{*}\right] \\
l & =3 \operatorname{Im}\left[\left(2 f_{M E}^{1+}-f_{M M}^{1-}\right)\left(f_{M M}^{1+}\right)^{*}\right] \\
m & =18 \operatorname{Im}\left[f_{E M}^{1+}\left(f_{M M}^{1+}\right)^{*}\right] .
\end{aligned}
$$

$$
\begin{aligned}
& \frac{d \bar{\sigma}}{d \Omega^{*}} \Sigma_{2 x}=\sin \theta\left(n+o \cos \theta+p \cos ^{2} \theta\right), \\
& \frac{d \bar{\sigma}}{d \Omega^{*}} \Sigma_{2 z}=q+r \cos \theta+s \cos ^{2} \theta+t \cos ^{3} \theta
\end{aligned}
$$

where

$$
\begin{aligned}
n & =-3\left|f_{M M}^{1+}\right|^{2}+3 \operatorname{Re}\left[\left(f_{M M}^{1-}+2 f_{M E}^{1+}\right)\left(f_{M M}^{1+}\right)^{*}\right] \\
o & =-3 \operatorname{Re}\left[\left(2 f_{E E}^{1+}+f_{E E}^{1-}-f_{E M}^{1+}\right)\left(f_{M M}^{1+}\right)^{*}\right] \\
p & =-18 \operatorname{Re}\left[f_{M E}^{1+}\left(f_{M M}^{1+}\right)^{*}\right] \\
q & =\left|f_{M M}^{1+}\right|^{2}+\operatorname{Re}\left[\left(f_{M M}^{1-}+6 f_{M E}^{1+}\right)\left(f_{M M}^{1+}\right)^{*}\right] \\
r & =2 \operatorname{Re}\left[\left(4 f_{E E}^{1+}+2 f_{E E}^{1-}+3 f_{E M}^{1+}\right)\left(f_{M M}^{1+}\right)^{*}\right] \\
s & =3\left|f_{M M}^{1+}\right|^{2}-3 \operatorname{Re}\left[\left(f_{M M}^{1-}-6 f_{E M}^{1+}\right)\left(f_{M M}^{1+}\right)^{*}\right] \\
t & =18 \operatorname{Re}\left[f_{E M}^{1+}\left(f_{M M}^{1+}\right)^{*}\right] .
\end{aligned}
$$

In analogy to Eq. (4.64) $\left|f_{M M}^{1+}\right|$ is found from the polarized differential cross section $d \sigma_{z}^{L} / d \Omega^{*}$ as follows:

$$
\left|f_{M M}^{1+}\right|=\sqrt{\frac{1}{3}(a-q)}=\sqrt{\left(\frac{d \sigma_{z}^{L}}{d \Omega^{*}}\right)_{\pi / 2}} .
$$




\subsection{LET of the observables in the c.m. system}

In analogy with the case of the invariant amplitudes we can decompose the observables $d \bar{\sigma} / d \Omega^{*}, \Sigma_{3}, \Sigma_{2 x}$ and $\Sigma_{2 z}$, which do not vanish below pion threshold, into the Born and the non-Born parts. The low energy expansion of the Born parts of the observables are given by the $A_{i}^{B}$ in Eq. (2.36) whose expansions up to $1 / \omega^{2}$ lead to

$$
\begin{gathered}
A_{1}^{B}=\frac{2 \pi r_{0} q^{2}}{\omega^{2}}, \quad A_{2}^{B}=-\frac{2 \pi r_{0}\left(q \kappa+q^{2}\right)}{\omega^{2}}, \quad A_{4}^{B}=-\frac{\pi r_{0} \kappa^{2}}{\omega^{2}}, \\
A_{3}^{B}=A_{5}^{B}=-\frac{\pi r_{0}\left(\kappa^{2}+2 q \kappa\right)}{\omega^{2}}, \quad A_{6}^{B}=\frac{\pi r_{0}\left(\kappa^{2}+2 q \kappa+2 q^{2}\right)}{\omega^{2}} .
\end{gathered}
$$

Here, $r_{0}=e^{2} / 4 \pi m \sim 1 / 137 m$. The non-Born parts of the $A_{i}$ in the limit of $\omega \rightarrow 0$, i.e. $A_{i}^{n B}(0,0)=a_{i}$, are deduced from Eqs. (2.43), (2.47) and (3.4) as follows:

$$
\begin{aligned}
& a_{1}=-2 \pi(\alpha-\beta) \\
& a_{2}=-4 \pi m\left(\gamma_{E 2}-\gamma_{M 2}\right) \\
& a_{3}=-2 \pi m\left(\gamma_{E 1}-\gamma_{E 2}+\gamma_{M 1}-\gamma_{M 2}\right)-2 \pi(\alpha+\beta), \\
& a_{4}=-2 \pi m\left(\gamma_{E 1}+\gamma_{E 2}+\gamma_{M 1}+\gamma_{M 2}\right), \\
& a_{5}=2 \pi m\left(\gamma_{E 1}+\gamma_{E 2}-\gamma_{M 1}-\gamma_{M 2}\right), \\
& a_{6}=2 \pi m\left(\gamma_{E 1}-\gamma_{E 2}+\gamma_{M 1}-\gamma_{M 2}\right) .
\end{aligned}
$$

We substitute the low energy expansion of the $A_{i}$, presented by the sum of Eq. (4.76) and (4.77), into the Eqs. (4.39), (4.41), (4.51) and (4.53), and we subtract then the resulting Born terms of the observables. We get then the non-Born parts of the observables represented up to $O\left(\omega^{4}\right)$ as follows:

$$
\begin{aligned}
{\left[\frac{d \bar{\sigma}}{d \Omega^{*}}\right]^{n B}=- } & r_{0} q^{2} m\left\{m\left(\left(1+z^{2}\right) \alpha+2 z \beta\right) \omega^{2}+2(1+z)^{2}(\alpha+\beta) \omega^{3}\right\} \\
& +\frac{m^{2}}{2}\left\{\left(1+z^{2}\right)\left(\alpha^{2}+\beta^{2}\right)+4 z \alpha \beta\right\} \omega^{4} \\
& -\frac{r_{0}}{8}\left\{\left(\kappa^{2}+2 q \kappa\right)(-1+z)\left(\left(3+z^{2}\right) \alpha-(1-z(4+z)) \beta\right)\right. \\
& \left.+2 q^{2}\left(\left(17+29 z+(19-z) z^{2}\right) \alpha+(3+z)(5+(10+z) z) \beta\right)\right\} \omega^{4} \\
- & \frac{r_{0}}{4} m\left\{q ( - 1 + z ) \left[-4 \kappa\left\{(-1+z) \gamma_{E 1}+(3+z) \gamma_{M 1}\right.\right.\right. \\
& \left.\quad-(1+(2-z) z) \gamma_{E 2}+2 z \gamma_{M 2}\right\} \\
& \left.\left.-(1-(2-5 z) z) \gamma_{E 2}+(3-(6+z) z) \gamma_{M 2}\right\}\right] \\
& \left.+\kappa^{2}\left[4 z\left(\gamma_{E 1}+\gamma_{M 2}\right)+2\left(3-z^{2}\right) \gamma_{M 1}-2\left(1-3 z^{2}\right) \gamma_{E 2}\right]\right\} \omega^{4}
\end{aligned}
$$




$$
\begin{aligned}
& {\left[\frac{d \bar{\sigma}}{d \Omega^{*}} \Sigma_{3}\right]^{n B}=r_{0} q^{2} m\left(1-z^{2}\right)\left(m \alpha \omega^{2}+2 \alpha \omega^{3}\right)-\frac{m^{2}}{2}\left(1-z^{2}\right)\left(\alpha^{2}-\beta^{2}\right) \omega^{4}} \\
& -\frac{r_{0}}{8}\left(1-z^{2}\right)\left\{2 q^{2}[(-9+z) \alpha-(1+z) \beta]\right. \\
& \left.-\left(\kappa^{2}+2 q \kappa\right)[(-3+z) \alpha-(1+z) \beta]\right\} \omega^{4} \\
& -\frac{r_{0}}{4} m\left(1-z^{2}\right)\left\{2\left(\kappa^{2}+2 q \kappa\right)\left(\gamma_{M 1}-\gamma_{E 2}\right)\right. \\
& \left.+q^{2}(-1+z)\left(\gamma_{E 1}-\gamma_{E 2}+\gamma_{M 1}-\gamma_{M 2}\right)\right\} \omega^{4} \\
& {\left[\frac{d \bar{\sigma}}{d \Omega^{*}} \Sigma_{2 x}\right]^{n B}=-\frac{r_{0}}{2} m \sqrt{1-z^{2}}\left\{\left(-(\kappa+q)^{2}+\left(2 q \kappa+q^{2}\right) z\right) \alpha\right.} \\
& +\left(q \kappa-(\kappa+q)\left(\kappa+q-q^{2} z\right) z\right) \beta \\
& \left.+2 m q^{2}\left(\gamma_{M 1}+z\left(\gamma_{E 1}+\gamma_{M 2}+z \gamma_{E 2}\right)\right)\right\} \omega^{3} \\
& +\frac{r_{0}}{4} \sqrt{1-z^{2}}\left\{( 1 + z ) \left[\left(5 \kappa^{2}-q(5 \kappa+3 q)(-1+z)\right) \alpha\right.\right. \\
& \left.+\left(\kappa^{2}(3+2 z)+\left(q \kappa+3 q^{2}\right)(1-z)\right) \beta\right] \\
& +2 m\left[\left(2 \kappa^{2}-2 q \kappa(-2+z)-q^{2}(1+(5+z) z)\right) \gamma_{E 1}\right. \\
& +\left(2 \kappa^{2} z-q \kappa(1+(-4+z) z)-q^{2}(4+(3+z) z)\right) \gamma_{M 1} \\
& +q\left(\kappa-5 q z+(\kappa-3 q) z^{2}\right) \gamma_{E 2} \\
& +\left(\kappa^{2}\left(-1+z^{2}\right)+2 q \kappa\left(-1+z+z^{2}\right)\right. \\
& \left.\left.\left.-q^{2}(3+(3+2 z) z)\right) \gamma_{M 2}\right]\right\} \omega^{4} \\
& {\left[\frac{d \bar{\sigma}}{d \Omega^{*}} \Sigma_{2 z}\right]^{n B}=-\frac{r_{0}}{2} m\left\{\left(q \kappa-2(\kappa+q)^{2} z+q(3 \kappa+2 q) z^{2}\right) \alpha\right.} \\
& -\left((\kappa+q)\left((\kappa+q)\left(1+z^{2}\right)-q z^{3}\right)-\left(3 q \kappa+q^{2}\right) z\right) \beta \\
& \left.+2 m q^{2}\left[\left(1+z^{2}\right)\left(\gamma_{E 1}+z \gamma_{E 2}\right)+2 z\left(\gamma_{M 1}+z \gamma_{M 2}\right)\right]\right\} \omega^{3} \\
& -\frac{r_{0}}{4}(1+z)\{[q \kappa(-1+z)((1+5 z) \alpha+(5+z) \beta) \\
& +3 q^{2}\left(-1+z^{2}\right)(\alpha+\beta)-\kappa^{2}(5 \beta-(3+2 z)(\alpha+z \beta) \\
& +2 m\left[\kappa ( - 1 + z ) \left\{\kappa\left(\gamma_{E 2}-2 \gamma_{M 1}-z \gamma_{M 2}\right)\right.\right. \\
& +q\left((4-z) \gamma_{M 1}-\gamma_{E 1}\right. \\
& \left.\left.+(z-2) \gamma_{E 2}+(1+2 z) \gamma_{M 2}\right)\right\} \\
& +q^{2}\left(2\left(3+z+z^{2}\right) \gamma_{E 1}+(1+3(z+2) z) \gamma_{E 2}\right. \\
& \left.\left.\left.+(5+(4+z) z) \gamma_{M 1}+2(4+z) z \gamma_{M 2}\right)\right]\right\} \omega^{4}
\end{aligned}
$$

As shown in Eqs. (4.78) and (4.79), for the proton $(q=1)$ the observables $d \bar{\sigma} / d \Omega^{*}$ and $\frac{d \bar{\sigma}}{d \Omega^{*}} \Sigma_{3}$ in an order $O\left(\omega^{2}\right)$ are dependent only on the dipole electric $\alpha$ and magnetic 
$\beta$ polarizabilities, and spin polarizabilities $\gamma_{i}$ are exhibited just in a $O\left(\omega^{4}\right)$. In the case of the neutron $(q=0)$ both polarizabilities enter into an order $O\left(\omega^{4}\right)$ in these observables. The double observables of proton Compton scattering $\Sigma_{2 x}$ and $\Sigma_{2 z}$ in Eqs. (4.80) and (4.81) are dependent both on $\alpha, \beta$ and on the $\gamma_{i}$ in $O\left(\omega^{3}\right)$, whereas in the case of the neutron they are characterized by the polarizabilities $\alpha$ and $\beta$, and the spin polarizabilities play a role only just in the order of $O\left(\omega^{4}\right)$. In other words, the effects of the parameters $\gamma_{i}$ to $\Sigma_{2 x}$ and $\Sigma_{2 z}$ begin in the order of $O\left(\omega^{3}\right)$ in the case of the proton, and start with $O\left(\omega^{4}\right)$ in the case of the neutron.

As a result, with respect to $\alpha$ and $\beta$, the single polarized proton differential cross sections $d \sigma^{\|(\perp)} / d \Omega^{*}$ are more sensitive than the double polarization observables $d \sigma_{x, z}^{R(L)} / d \Omega^{*}$. In contrast to the proton, for the neutron the sensitivity to $\alpha$ and $\beta$ can be observed better in the $d \sigma_{x, z}^{R(L)} / d \Omega^{*}$ than in the $d \sigma^{\|(\perp)} / d \Omega^{*}$. With reference to the spin polarizabilities $\gamma_{i}$, the $d \sigma_{x, z}^{R(L)} / d \Omega^{*}$ for the proton is most respone to a change of the value of $\gamma_{i}$ compared with other observables.

At the three c.m. angles $\theta=0, \pi / 2$ and $\pi$, the low energy approximations of the observables take particually simple forms.

- At the forward scattering $\theta=0$ :

$$
\begin{aligned}
& {\left[\frac{d \bar{\sigma}}{d \Omega^{*}}\right]_{\theta=0}^{n B}=}-2 r_{0} q^{2} m\left\{m(\alpha+\beta) \omega^{2}+(\alpha+\beta) \omega^{3}\right\} \\
&+\left\{m^{2}(\alpha+\beta)^{2}-16 r_{0} q^{2}(\alpha+\beta)-r_{0} m \kappa \gamma_{0}\right\} \omega^{4} \\
& {\left[\frac{d \bar{\sigma}}{d \Omega^{*}} \Sigma_{2 z}\right]_{\theta=0}^{n B}=-\frac{r_{0}}{2} m\left\{\left(q \kappa-2(\kappa+q)^{2}+q(3 \kappa+2 q)\right)(\alpha+\beta)+q^{2} \beta\right\} \omega^{3} } \\
&+4 r_{0} m q^{2} \gamma_{0} \omega^{3}+5 r_{0}\left(\kappa^{2}(\alpha+\beta)+2 m q^{2} \gamma_{0}\right) \omega^{4}
\end{aligned}
$$

- At the backward scattering $\theta=\pi$ :

$$
\begin{aligned}
& {\left[\frac{d \bar{\sigma}}{d \Omega^{*}}\right]_{\theta=\pi}^{n B}=}-2 r_{0}^{2} q^{2} m^{2}(\alpha-\beta) \omega^{2} \\
&+\left\{m^{2}(\alpha-\beta)^{2}+r_{0}\left(\kappa^{2}+2 q \kappa-q^{2}\right)(\alpha-\beta)\right\} \omega^{4} \\
&+r_{0} m\left\{4 q^{2}\left(\gamma_{E 2}-\gamma_{M 2}\right)+\left(\kappa^{2}+4 q \kappa\right) \gamma_{\pi}\right\} \omega^{4}, \\
& {\left[\frac{d \bar{\sigma}}{d \Omega^{*}} \Sigma_{2 z}\right]_{\theta=\pi}^{n B}=-\frac{r_{0}}{2} m\left\{\left[q \kappa+2(\kappa+q)^{2}+q(3 \kappa+2 q)\right](\alpha-\beta)\right.} \\
&\left.-q^{2} \beta-4 m q^{2} \gamma_{\pi}\right\} \omega^{3}
\end{aligned}
$$

As shown in the Eqs. (4.83) and (4.85), the c.m. polarized differential cross section $d \sigma_{z}^{R(L)} / d \Omega^{*}$ of Compton scatteing depends on $\alpha+\beta$ and $\gamma_{0}$ at the forward angle and on $\alpha-\beta$ and $\gamma_{\pi}$ at the backward angle, respectively. For the neutron the expansion of $\Sigma_{2 z}$ up to $O\left(\omega^{4}\right)$ is independent of the spin polarizabilities at these two special angles. 
- At the angle of $\theta=\frac{\pi}{2}$ :

$$
\begin{aligned}
& {\left[\frac{d \bar{\sigma}}{d \Omega^{*}}\right]_{\theta=\pi / 2}^{n B}=-r_{0} q^{2} m\left\{m \alpha \omega^{2}+2(\alpha+\beta) \omega^{3}\right\}+\frac{m^{2}}{2}\left(\alpha^{2}+\beta^{2}\right) \omega^{4}} \\
& +\frac{r_{0}}{8}\left\{\left(\kappa^{2}+2 q \kappa\right)(3 \alpha-\beta)-q^{2}(34 \alpha+30 \beta)\right\} \omega^{4} \\
& -\frac{r_{0}}{2} m\left\{\kappa^{2}\left(\gamma_{E 2}-3 \gamma_{M 1}\right)+2 q \kappa\left(\gamma_{E 1}+\gamma_{E 2}-3 \gamma_{M 1}\right)\right. \\
& \left.-q^{2}\left(3 \gamma_{E 1}+\gamma_{E 2}+3 \gamma_{M 1}-3 \gamma_{M 2}\right)\right\} \omega^{4}, \\
& {\left[\frac{d \bar{\sigma}}{d \Omega^{*}} \Sigma_{3}\right]_{\theta=\pi / 2}^{n B}=r_{0} q^{2} m\left(m \alpha \omega^{2}+2 \alpha \omega^{3}\right)-\frac{1}{2} m^{2}\left(\alpha^{2}-\beta^{2}\right) \omega^{4}} \\
& -\frac{r_{0}}{8}\left\{\left(\kappa^{2}+2 q \kappa\right)(3 \alpha+\beta)-2 q^{2}(9 \alpha+\beta)\right\} \omega^{4} \\
& -\frac{r_{0}}{4} m\left\{2 \kappa(\kappa+2 q)\left(-\gamma_{E 2}+\gamma_{M 1}\right)\right. \\
& \left.\left.+q^{2}\left(-\gamma_{E 1}+\gamma_{E 2}-\gamma_{M 1}+\gamma_{M 2}\right)\right]\right\} \omega^{4}, \\
& {\left[\frac{d \bar{\sigma}}{d \Omega^{*}} \Sigma_{2 x}\right]_{\theta=\pi / 2}^{n B}=-\frac{r_{0}}{2} m\left\{q \kappa \alpha-(\kappa+q)^{2} \beta\right\} \omega^{3}-2 r_{0} q^{2} m^{2} \gamma_{M 1} \omega^{3}} \\
& +\frac{r_{0}}{4}\left\{\left(5 \kappa^{2}+5 q \kappa+3 q^{2}\right) \alpha+\left(3 \kappa^{2}+3 q \kappa+q^{2}\right) \beta\right\} \omega^{4} \\
& -\frac{r_{0}}{2} m\left\{\kappa(\kappa+2 q)\left(-2 \gamma_{E 1}+\gamma_{M 2}\right)+q \kappa\left(-\gamma_{E 2}+\gamma_{M 1}\right)\right. \\
& \left.+q^{2}\left(\gamma_{E 1}+4 \gamma_{M 1}+3 \gamma_{M 2}\right)\right\} \omega^{4} \\
& {\left[\frac{d \bar{\sigma}}{d \Omega^{*}} \Sigma_{2 z}\right]_{\theta=\pi / 2}^{n B}=-\frac{r_{0}}{2} m\left\{q \kappa \alpha-(\kappa+q)^{2} \beta\right\} \omega^{3}-r_{0} q^{2} m^{2} \gamma_{E 1} \omega^{3}} \\
& +\frac{r_{0}}{4}\left\{\left(3 \kappa^{2}+q \kappa+3 q^{2}\right) \alpha+\left(5 \kappa^{2}+5 q \kappa+3 q^{2}\right) \beta\right\} \omega^{4} \\
& -\frac{r_{0}}{2} m\left\{\kappa(\kappa+2 q)\left(2 \gamma_{M 1}-\gamma_{E 2}\right)+q \kappa\left(-\gamma_{E 1}+\gamma_{M 2}\right)\right. \\
& \left.+q^{2}\left(6 \gamma_{E 1}+\gamma_{E 2}+5 \gamma_{M 2}\right)\right\} \omega^{4} \text {. }
\end{aligned}
$$

At the angle $\theta=\pi / 2$ the proton polarized cross sections $d \sigma_{z}^{R(L), p} / d \Omega^{*}$ depend primarily on $\gamma_{M 1}$ and $\gamma_{E 2}$ as well as $\alpha$ and $\beta$ in an order of $O\left(\omega^{3}\right)$, while the neutron polarized cross sections $d \sigma_{z}^{R(L), n} / d \Omega^{*}$ in this order is dependent only on magnetic polarizability $\beta$. On the other hand, the $d \sigma^{\|(\perp), p} / d \Omega^{*}$ in $O\left(\omega^{3}\right)$ are described by the electromagnetic polarizabilities. The sensitivity of the neutron polarized cross sections $d \sigma^{\|(\perp), n} / d \Omega^{*}$ to $\beta$ appear just in $O\left(\omega^{4}\right)$. As a consequency, the neutron electromagnetic polarizability could be extracted better from the experiment using the circularly polarized photon and the neutron polarized in $\mathrm{x}$ or $\mathrm{z}$ direction at this angle. 


\subsection{Numerical results and Discussion}

In this section, we present numerical results for observables of polarized proton Compton scattering in comparision with the experimental data from Refs. [40, 41, 42, 26, 28, 32, 24, 2.9, 30, 23, 21, 44, 38, 22. 34, 46, 47].

The c.m. differential cross sections given in Fig. 4.7 at six different angles and energies up to $900 \mathrm{MeV}$ are, in general, in good agreement with the experimental data. Here, the unitarity bounds of the dashed lines are obtained by setting $\operatorname{Re} A_{i}=0$. The results for the photon $\Sigma_{3}$ at $\theta \lesssim 90^{\circ}$ and nucleon $P$ asymmetry at the c.m. angles $\theta \gtrsim 132^{\circ}$ show the deviations from the experimental values.

The dependence of the unpolarized differential cross sections and asymmetries on the parameters $(\alpha-\beta)^{p}$ and $\gamma_{\pi}^{p}$ are demonstrated in Figs. 4.9 4.11 and in Figs. 4.12-4.12, respectively, where the results with $(\alpha-\beta)^{p}=10.0$ and $\gamma_{\pi}^{p}=-37.1$ are plotted by means of the solid lines. The dashed and dotted curves of Figs. 4.9, 4.10 (left) and 4.11 are deduced from the two different values of $(\alpha-\beta)^{p}=12$ and $(\alpha-\beta)^{p}=7$ respectively, where the value for the proton backward spin polarizability is kept as -37.1. Analogously, the predictions for fixed $(\alpha-\beta)^{p}$ and variation of $\gamma_{\pi}^{p}$ between -39.6 and -25.1 are represented in Figs. 4.10 (right), 4.12 and 4.13 using the dashed and dotted lines, respectively. Here, the latter value of -25.1 is taken from the LEGS experiment [2, 53], and the former one of -39.6 is derived from the backward unsubtracted DR of Ref. [6.3].

Below pion threshold the dependence of the observables $d \bar{\sigma} / d \Omega^{*}, \Sigma_{3}, \quad \Sigma_{2 x}$ and $\Sigma_{2 z}$ upon the strength of both $\alpha-\beta$ and $\gamma_{\pi}$ is not very large. In particular, the asymmetry $\Sigma_{2 x}$ is almost insensitive to the change of polarizabilities. At energies up to $50 \mathrm{MeV}$ an effect on these parameters is barely observed. As so far, our results sustain the conclusions in Refs. [16, 15] and [17] drawn in the framework of fixed- $t$ unsubtracted and subtracted DR respectively, where in the latter reference the exploitations are done only at two extremely forward and backward angles, i.e. $\theta=0^{\circ}, 180^{\circ}$, and at $\theta=90^{\circ}$.

At higher energies $\left(150 \mathrm{MeV} \lesssim E_{\gamma} \lesssim 300 \mathrm{MeV}\right)$ the sensitivity of the observables to the difference of the electic and magnetic polarizabilities as well as the backward spin polarizability is, on the whole, increased evidently, indeed, in unpolarized differential cross section and $\Sigma_{2 z}$. As to the variation of $(\alpha-\beta)^{p}$, the differential cross sections show the minimal sensitivity at $\theta=90^{\circ}$, while the alterations of the proton backward spin polarizability carry a change in $d \bar{\sigma} / d \Omega^{*}$ scarcely at c.m. angles $\theta \lesssim 60^{\circ}$, see Figs. 4.9 and 4.12. In the case of $\Sigma_{3}$ and $\Sigma_{2 z}$, the parameters of the electromagnetic polarizabilities have a larger influence on this observables than on the spin polarizability $\gamma_{\pi}^{p}$. It is also recognized in Figs. 4.11 and 4.13 that the dependence of $\Sigma_{2 z}$ as well as $\Sigma_{2 x}$ on both polarizabilitites is decreased at $\theta \geq 150^{\circ}$. After all, the measurements of the observables in the region of energies above the pion threshold might be more adequate than the lower energies to provide some useful constraints on the values of the polarizabilities. It must, however, be remembered that above two-pion thresh- 
old $(\omega \geq 309 \mathrm{MeV})$ the model dependence rises strongly due to the not well-known $2 \pi$-photoproduction amplitude in the $s$-channel.

The effect of the mixing ratio $E 2 / M 1$ on the polarized observables involving the differential cross sections are investigated in Figs. 4.14-4.17, where the solid curves result from the VPI-SP98K parametrization with $E 2 / M 1=-1.6 \%$, which is obtained from fit to the Mainz data [8, 52$]$. Here, the mixing ratio is associated with $\operatorname{Im} E_{1+}^{3 / 2} / \operatorname{Im} M_{1+}^{3 / 2}$ at $\omega=340 \mathrm{MeV}$ with $\operatorname{Re} M_{1+}^{3 / 2} \rightarrow 0$. In this analysis, the background part is kept fixed, and only the resonant contribution to $M_{1+}^{3 / 2}$ or $E_{1+}^{3 / 2}$ is rescaled in the vicinity of $\Delta[\mathbf{7 4}, 433,8,52]$. This value agrees excellently with the value of $E 2 / M 1=-1.6 \pm 0.3_{\text {ind }} \pm 0.8_{\text {com }} \pm 0.1_{\text {mod }} \%$ derived lately from the data of the LARA experiment in Mainz [2.9]. We change the value of the $E 2 / M 1$ on the one hand to $-3 \%$ (dashed curves) found by the LEGS [27] and RPI [I.9] groups, on the other hand to the largest negative value from model $-5 \%$ (dotted curves). The finding based on SAID-SM95 in which the strength of $M_{1+}$ is reduced by $2.8 \%$, are represented by the dashed-dotted curves.

At the angles $\theta=75^{\circ}$ and $90^{\circ}$, the larger quadrupole amplitude $E 2$ has destructive effects to lower the $\Delta$-peak of cross sections, whereas at $\theta=135^{\circ}$ and $150^{\circ}$ the increased values of $E 2 / M 1$ cause a higher maximum of the cross section, see Fig. 4.14. In any case, the largest sensitivity of the differential cross section to $E 2 / M 1$ is given at $\omega \simeq 320 \mathrm{MeV}$ and, in particular, at larger backward angles. It seems that the curves from dispersive calculation by using old SAID solution VPI-SM95 improves the agreements with the data from [27] at $\theta=75^{\circ}$ and [2.9] at $\theta=135^{\circ}$. At $\theta=90^{\circ}$ all experimental points lie slightly above the results by DR, whereas at $\theta=150^{\circ}$ the measurements of [40, 41, [23, [2.9] are preferred to be described by an increased quadrupole strength. That is, a proper determination of the $E 2$ contribution requires more data over a wide angular range.

Double polarization asymmetries appear to be generally more sensitive than the beamand target-polarization asymmetries, $\Sigma_{3}$ and $P$, except for $\Sigma_{2 x}$ up to energies of the $\Delta$-peak. Especially, the beam-target asymmetry $\Sigma_{1 z}$ is, as shown in Fig. 4.17, remarkably responsive to the change of quadrupole amplitude $E 2$ in the relatively wide range of angles, i.e. $60^{0} \lesssim \theta \lesssim 90^{\circ}$ together with $\Sigma_{2 z}$ at the angles $\theta \lesssim 90^{\circ}$ and around $\omega \simeq 450 \mathrm{MeV}$. 

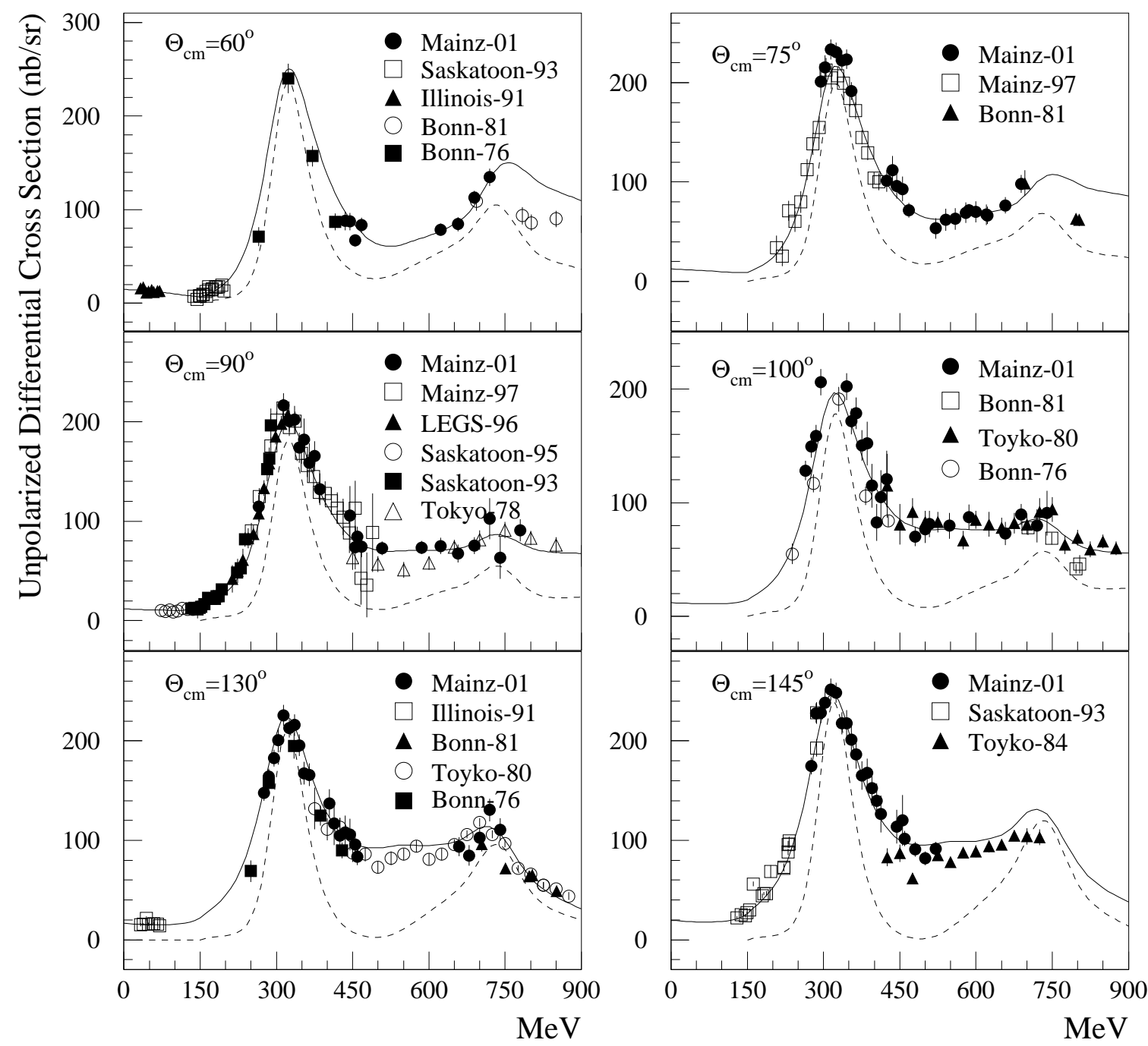

Figure 4.7.: Energy dependence of the c.m. unpolarized differential cross sections of proton Compton scattering at several angles. The solid lines represent the predictions of dispersive calculation using the pion multipole analysis of the VPI group [73, 74], while the dashed lines show the unitarity bound obtained from $\operatorname{Re} A_{i}=0$. The experimental data are from Refs. [28, [3\%, [24, [2.9, [30, [2:3, 201, 44, [38, 20, [34, 47]. 

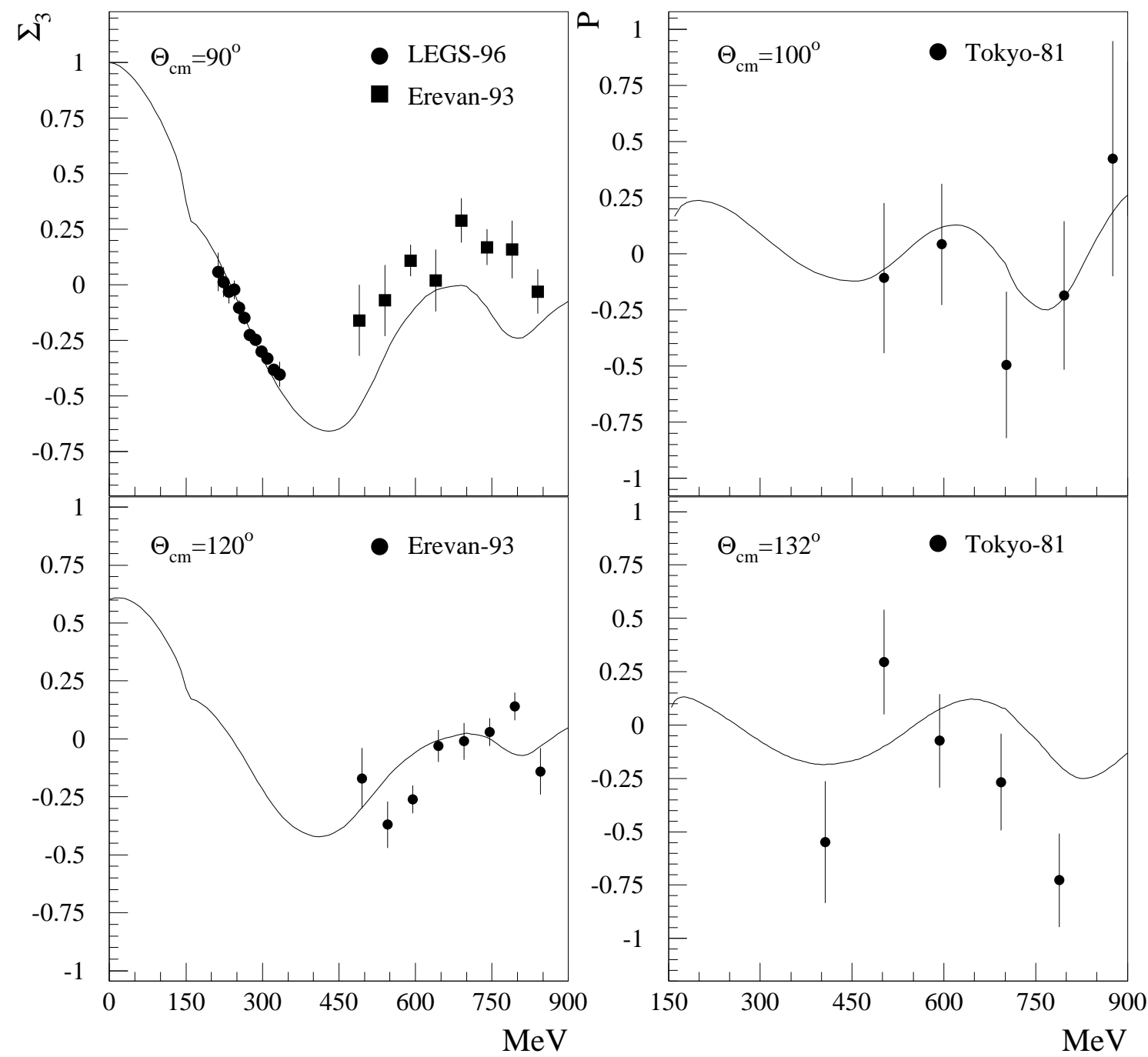

Figure 4.8.: Energy dependence of the photon $\Sigma_{3}$ (left) and the proton $P$ (right) asymmetry of the reaction $\vec{\gamma} p \rightarrow \gamma p$. The data are taken from Refs. [25, [28, 46]. 

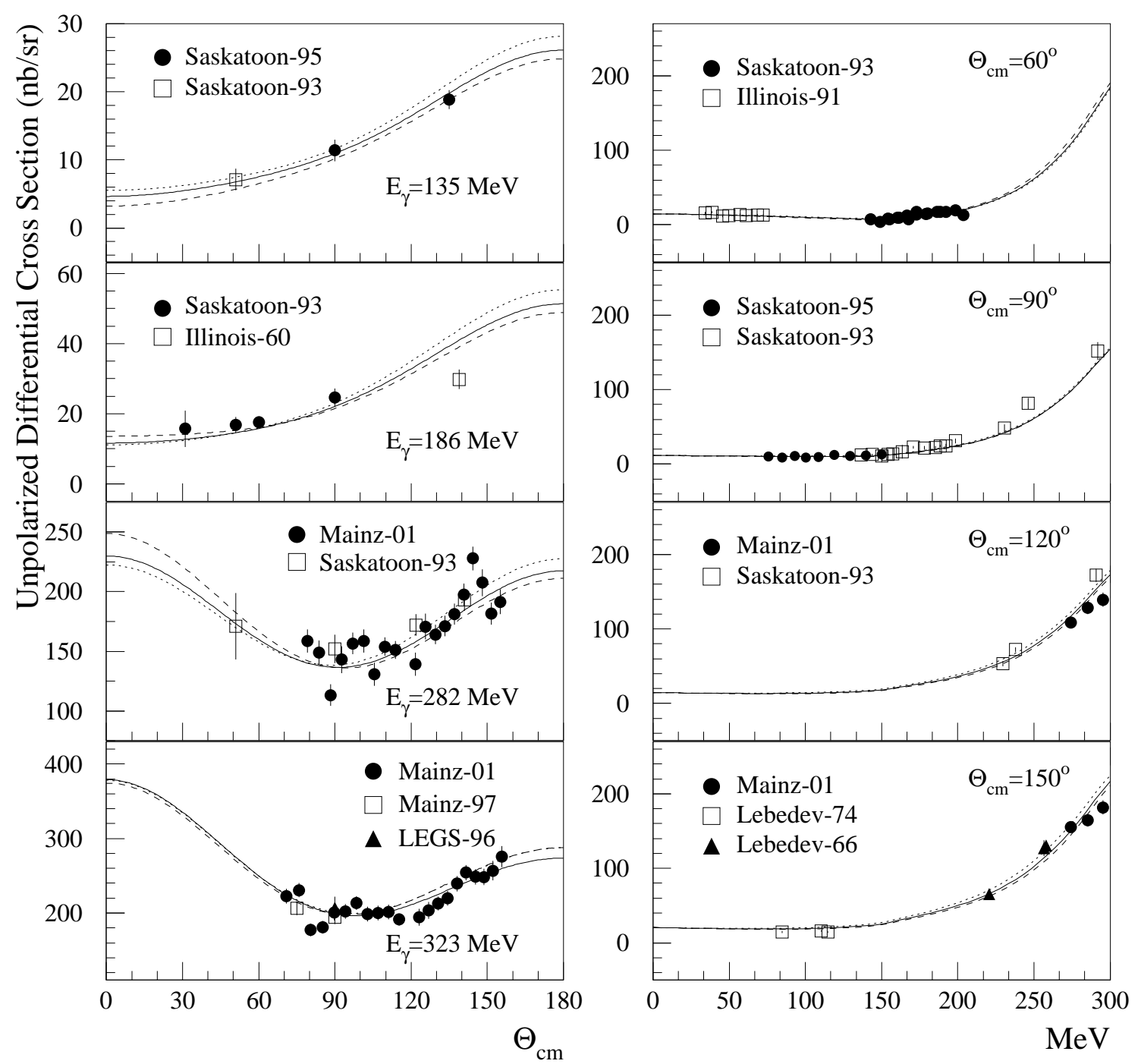

Figure 4.9.: Unpolarized differential cross sections at the energies $\omega=135,186,282$ and $323 \mathrm{MeV}$ (left) and at the four scattering angles $\theta=60^{\circ}, 90^{\circ}, 120^{\circ}$ and $150^{\circ}$ (right). The results from the difference bwteen the electric and magnetic polarizability $(\alpha-\beta)^{p}=10$ and proton backward spin polarizability $\gamma_{\pi}^{p}=-37.1$ are represented by the solid lines, while the predictions for fixed $\gamma_{\pi}^{p}$ and two different values of $\alpha-\beta=12$ and $(\alpha-\beta)^{p}=7$ are displayed using the dashed and dotted lines, respectively. The data are from Refs. [40, 41, [26, [28, [24, [29, [2:], [21, [20] . 

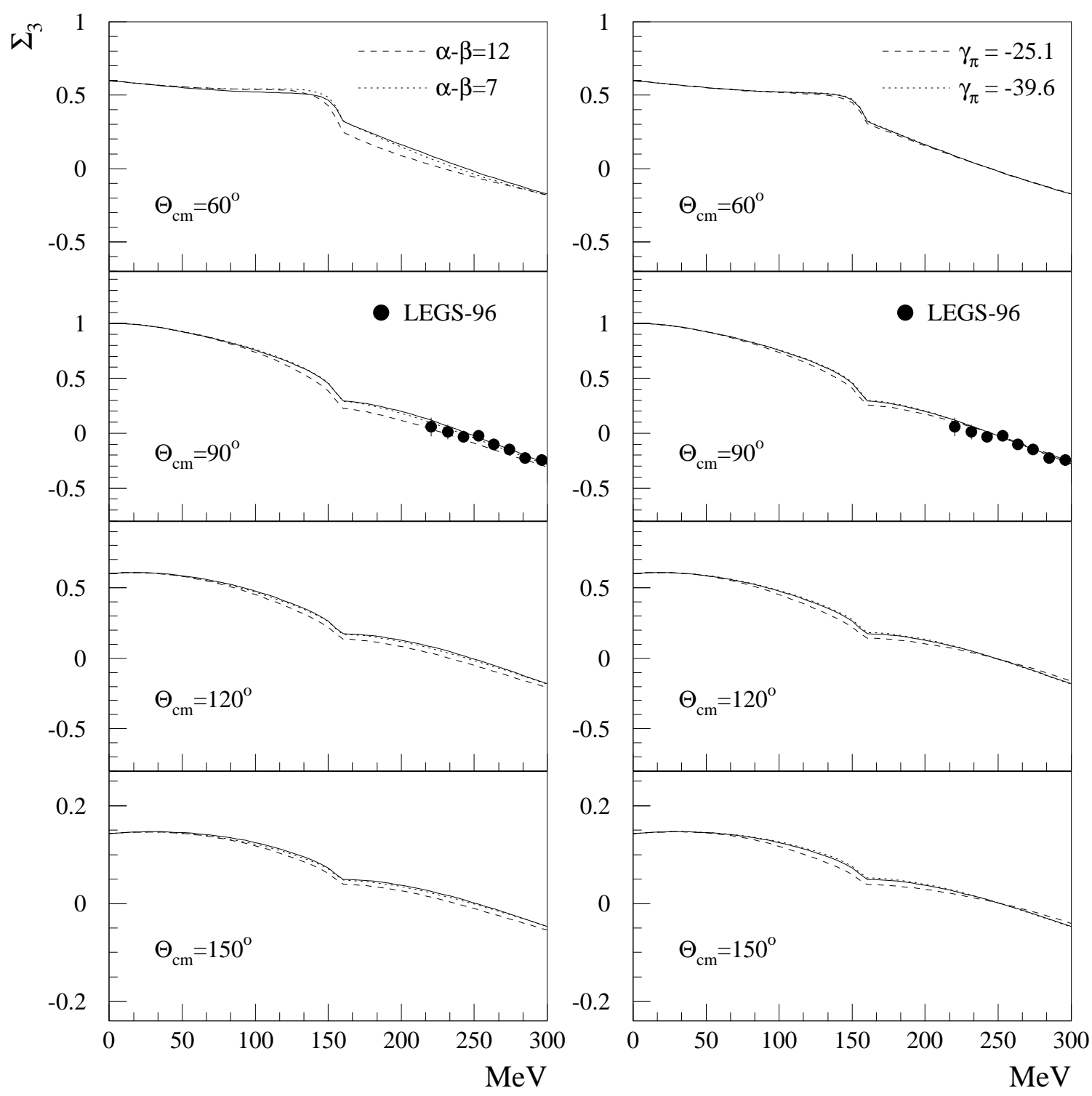

Figure 4.10.: Photon asymmetries $\Sigma_{3}$ at the angles of $\theta_{c m}=60^{\circ}, 90^{\circ}, 120^{\circ}$ and $150^{\circ}$. The predictions for a fixed $\gamma_{\pi}^{p}$ and the different $(\alpha-\beta)^{p}=$ 12, 7 are presented at the left subfigures by the dashed and dotted lines, respectively, whereas that for a fixed $(\alpha-\beta)^{p}$ and $\gamma_{\pi}^{p}=$ -25.1 (dashed lines), -39.6 (dotted lines) is shown at the right subfigures. The data are from Ref. [28]. 

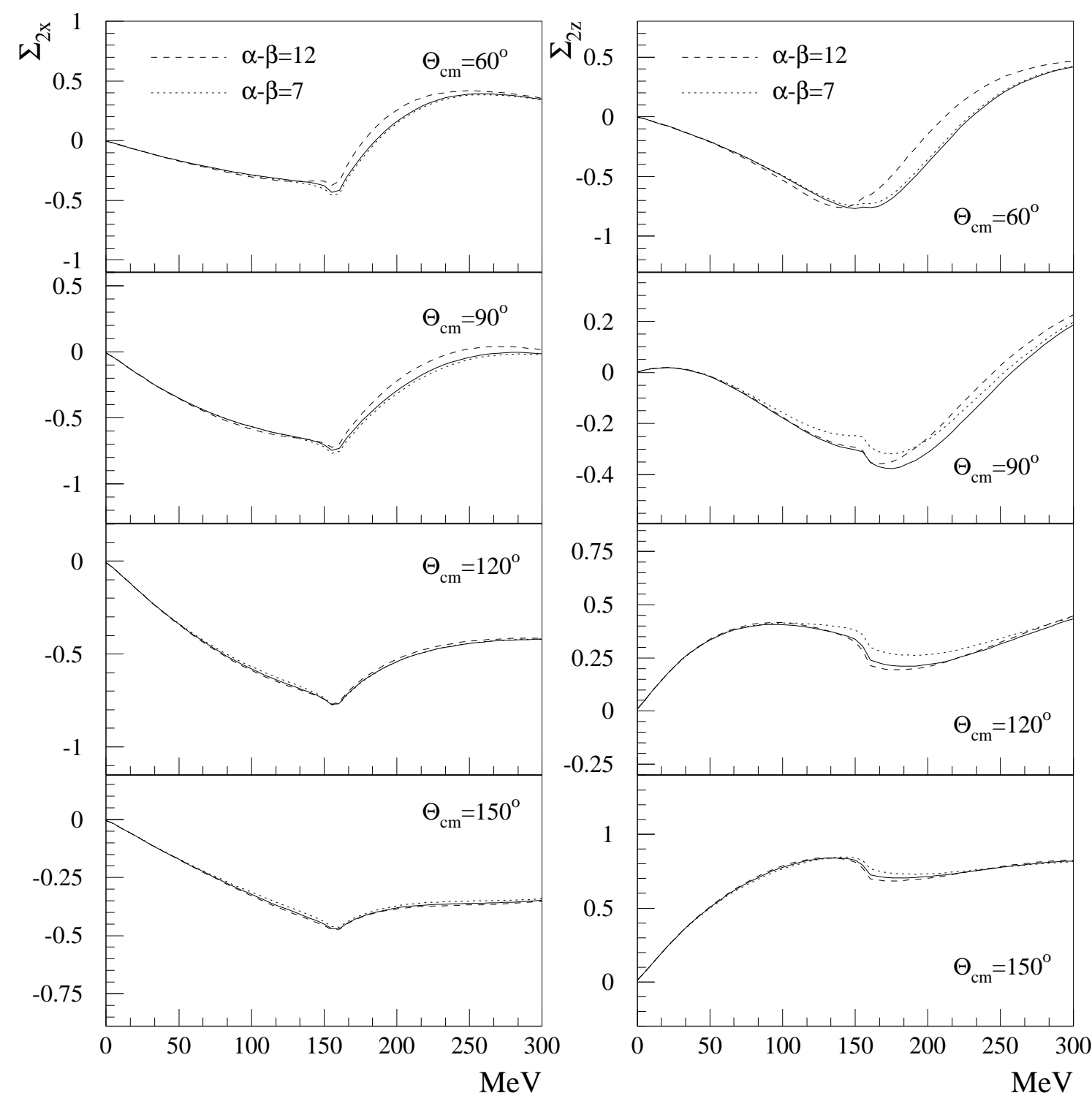

Figure 4.11.: Asymmetries $\Sigma_{2 x}$ (left) and $\Sigma_{2 z}$ (right) of Compton scattering by the circularly polarized photon on the proton polarized in the $x$ and $z$ directions, respectively. Notations are the same as explained in Fig. 4.9 . 

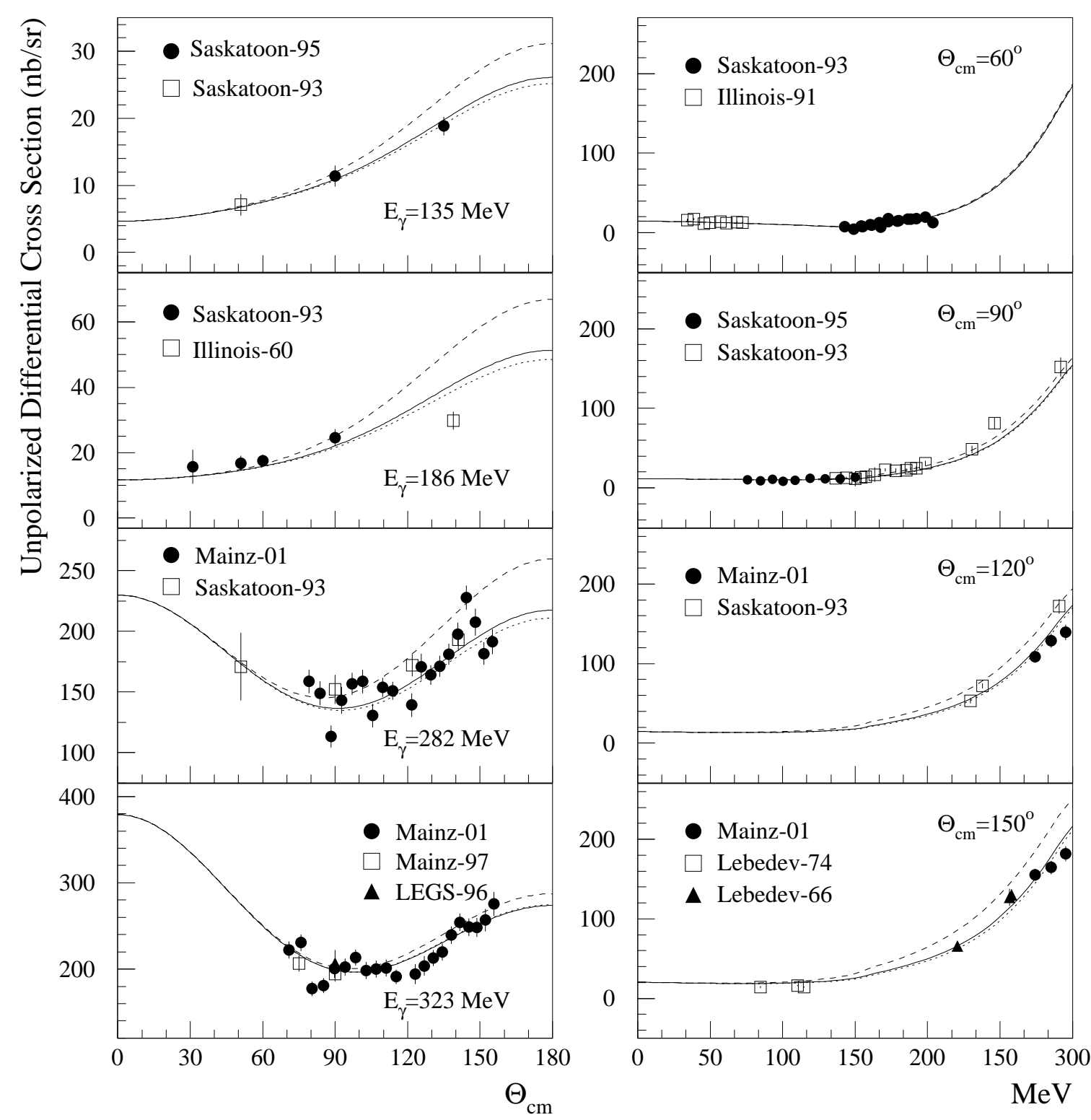

Figure 4.12.: Unpolarized differential cross sections with respect to the angles (left) and the energies (right) for the fixed $(\alpha-\beta)^{p}$ and different $\gamma_{\pi}^{p}=$ -25.1 (dashed lines) and -39.6 (dotted lines). The solid lines show the results from $(\alpha-\beta)^{p}=10$ and $\gamma_{\pi}^{p}=-37.1$. Data from [40, 41, 40, [28, [24, [2.9, [2:3, [21, [22]. 

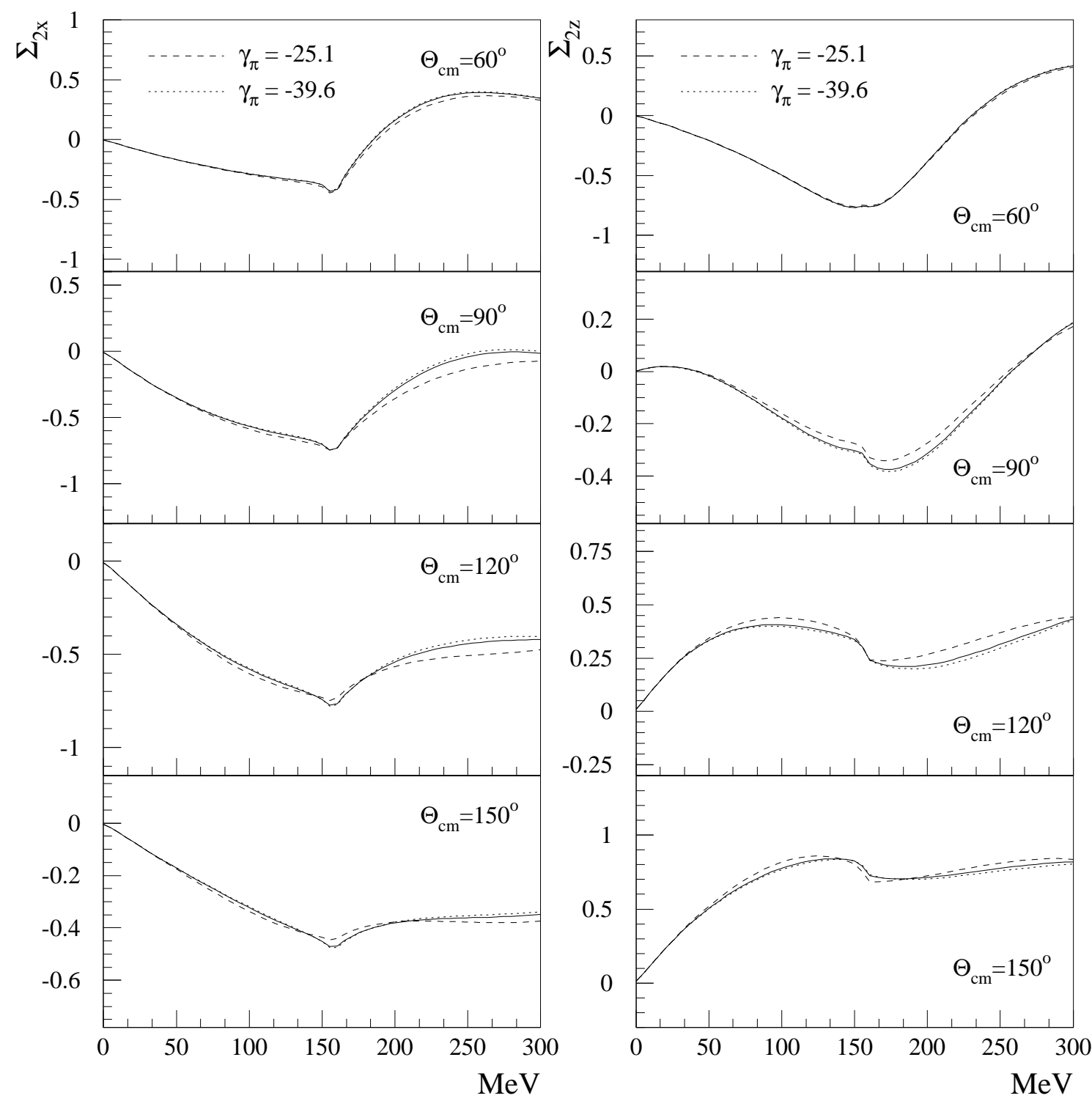

Figure 4.13.: Dependence of the asymmetries $\Sigma_{2 x}$ (left) and $\Sigma_{2 z}$ (right) on the backward spin polarizability $\gamma_{\pi}$. Notations are the same as described in Fig. 座.19. 

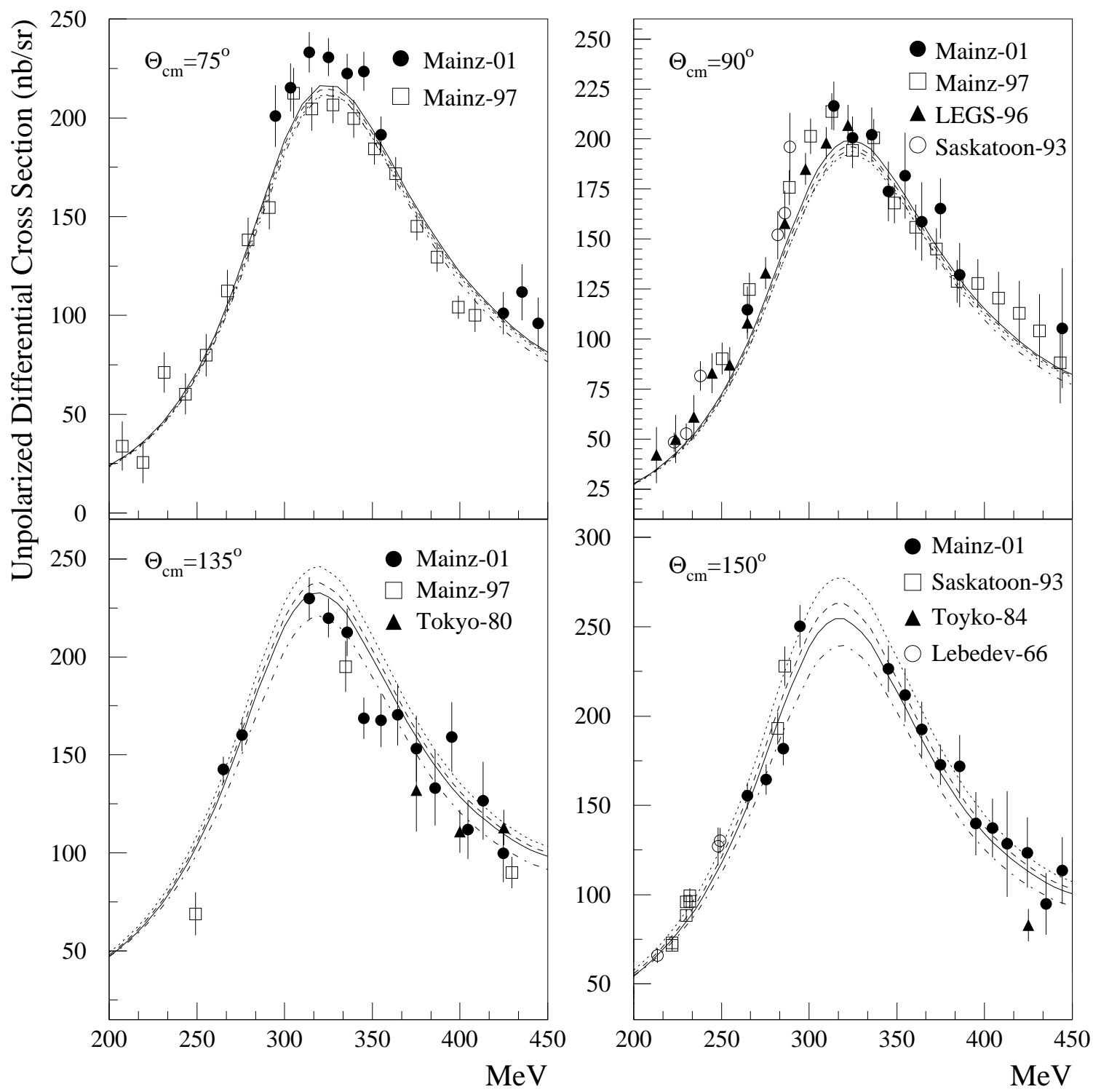

Figure 4.14.: Unpolarized differential cross sections of the Compton scattering off the proton for the different E2/M1 ratio. The solid lines demonstrate the results with $E 2 / M 1=-1.6 \%$. The predictions for the $E 2 / M 1=-3 \%$ and $-5 \%$ are illustrated by means of the dashed and dotted lines respectively. The dashed-dotted lines represent the values using the solution VPI-SM95 in which the strength of the amplitude $M_{1+}$ is reduced by 2.8\%. Data are taken from [40, 41, [28, [2.9, [2.], [21, 44, 47] 

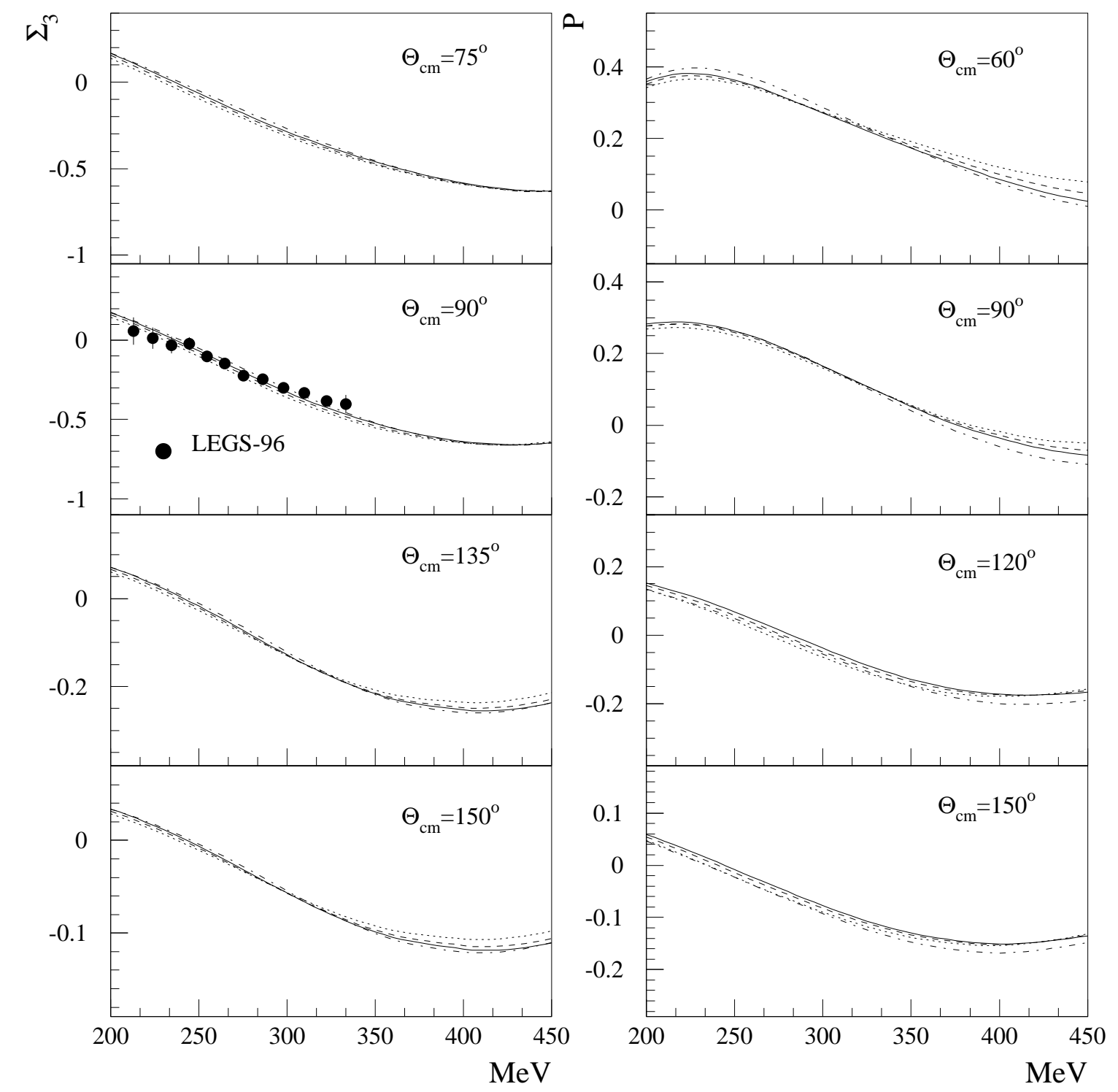

Figure 4.15.: Dependence of the beam $\Sigma_{3}$ and proton asymmetry $P$ on the parameter $E 2 / M 1$ in the region of $\Delta$-resonance. The notations are the same as described in Fig. 4.14. 

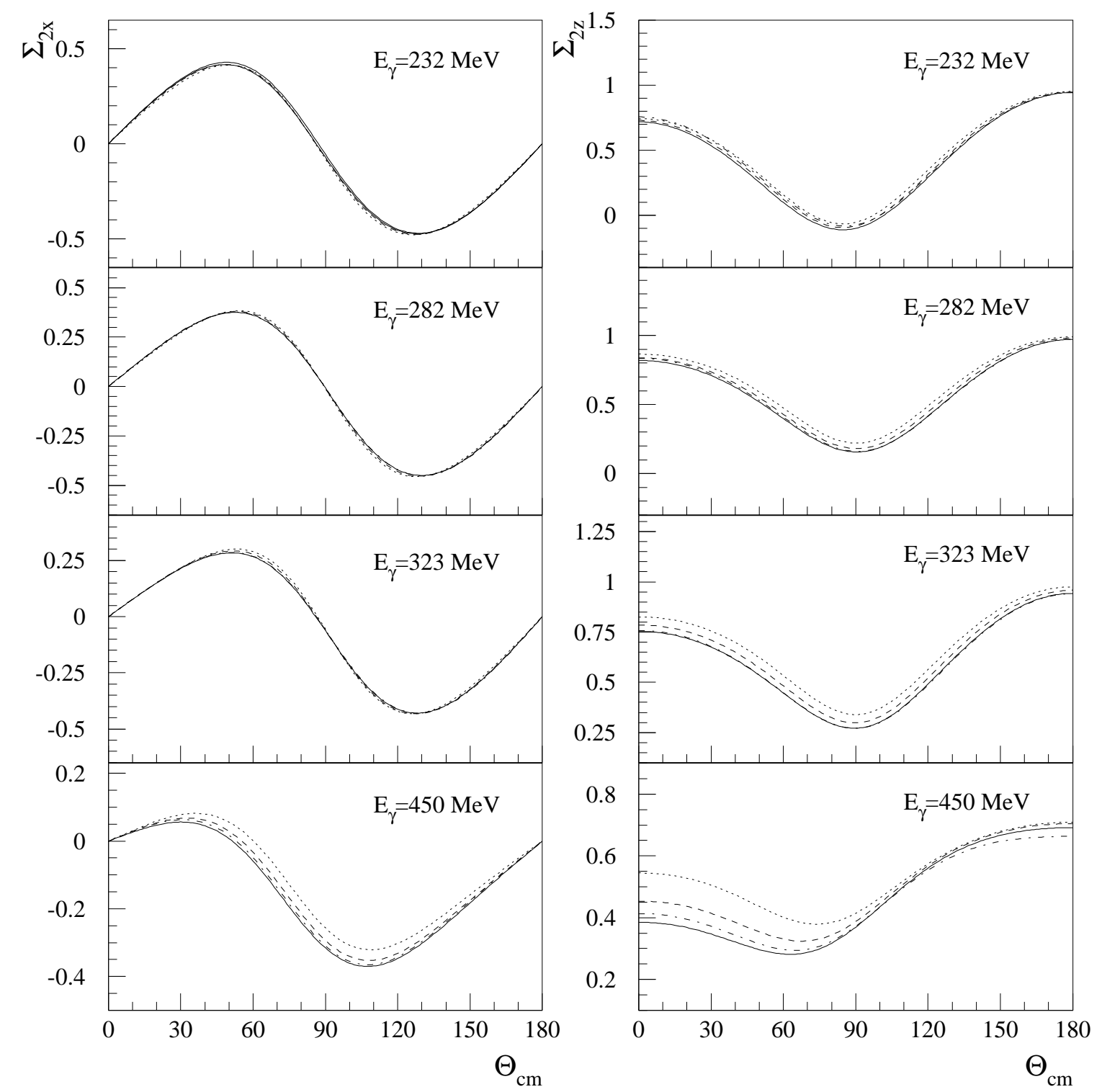

Figure 4.16.: Sensitivity of the asymmetries $\Sigma_{2 x}$ (left) and $\Sigma_{2 z}$ (right) to the mixing ratio of E2/M1. The notations are the same as described in Fig. 4.14. 

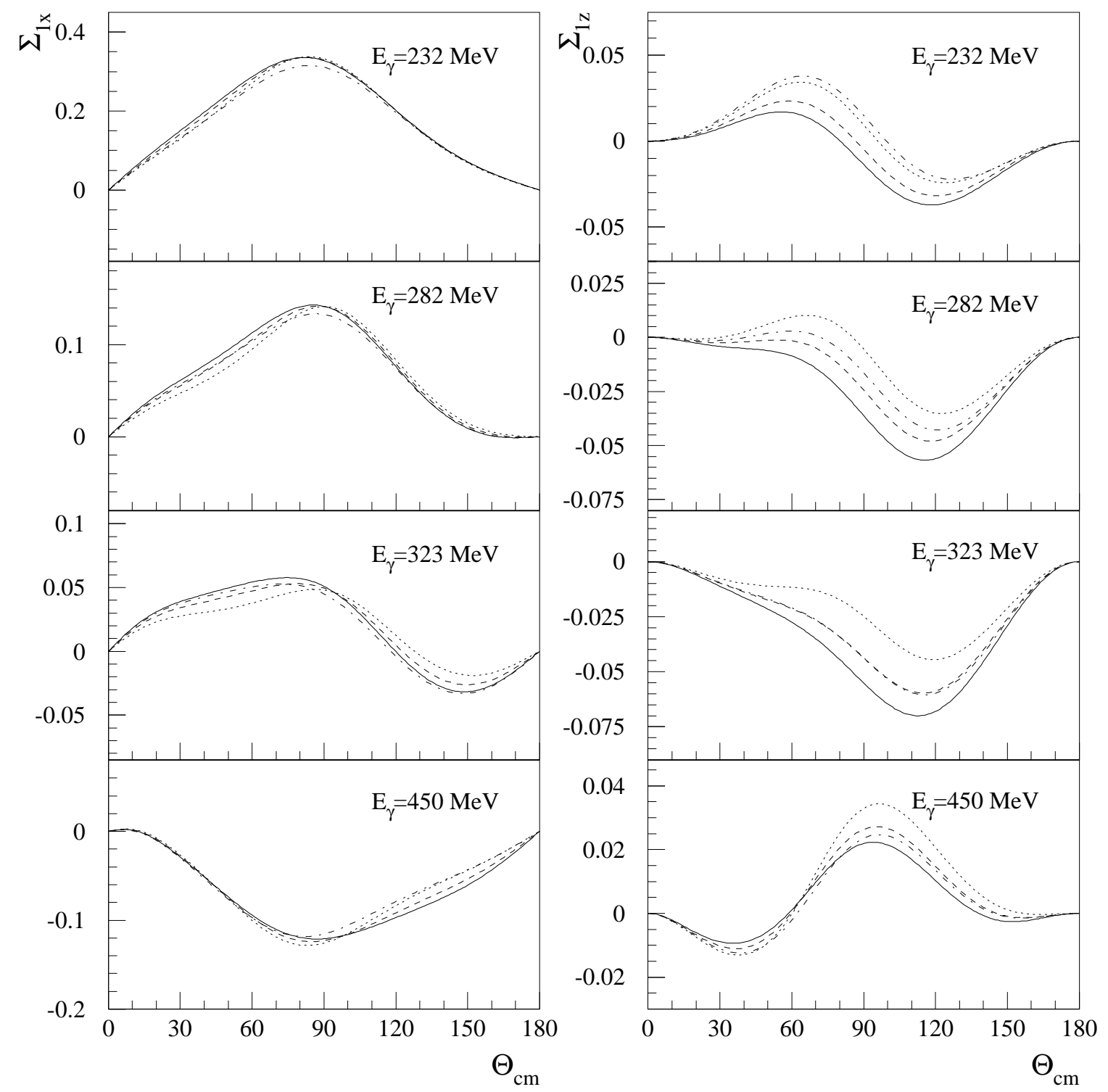

Figure 4.17.: Sensitivity of the asymmetries $\Sigma_{1 x}$ (left) and $\Sigma_{1 z}$ (right) to the E2/M1. The same notations as in Fig. [4.14. 


\section{Summery}

The differential cross sections and the single and double asymmetries for the nucleon Compton scattering in the first and second resonance regions have been calculated within the fixed- $t$ unsubtracted dispersion relation by using VPI-SP98K parametrization of the photo-meson amplitudes. The dependence of these observables on the difference of the electric and magnetic polarizabilities of the proton, $(\alpha-\beta)^{p}$, the proton backward spin polarizability $\gamma_{\pi}^{p}$ as well as the multipole mixing ratio $E 2 / M 1$ of $p \rightarrow \Delta$ has been also investigated, in order to provide more information on these quantities.

The structure parameters $\alpha, \beta$ and $\gamma_{\pi}$, which describe the non-Born part of Compton scattering amplitude in the limit of $\omega \rightarrow 0$ on the basis of low energy expansion, have been expressed by the invariant amplitudes $A_{1} A_{2}$ and $A_{5}$, where the asymptotic parts of the $A_{1}$ and the $A_{2}$ are saturated by the $t$-channel exchange of $\sigma$-meson and $\pi^{0}$-meson respectively. The proton backward spin polarizability $\gamma_{\pi}^{p}=-37.1$ obtained by DR has agreed well both with the prediction of heavy baryon ChPT and with the latest results from the LARA [29] and the TAPS [20] experiments in Mainz. The contradiction to the analysis of the LEGS [2, 5:3] group might originate, as refered in Ref. [2.9], from the disagreement of the measured cross sections above $\pi$-threshold, mainly at backward angles. In general, the dependence of the polarizabilities to the differential cross sections and the asymmetries has been clearly visible at the energies above pion threshold. Indeed, $d \bar{\sigma} / d \Omega^{*}$ at large angles and $\Sigma_{2 z}$ at $\theta \lesssim 150^{\circ}$ have displayed an evidently large sensitivity to the $\alpha-\beta$ and $\gamma_{\pi}$. As a consequence, the measurement of the observable at the energies up to two $\pi$-threshold and at mainly large angles is adequate to constrain the value of the polarizabilities.

With respect to the variation of $E 2 / M 1$ the unpolarized differential cross section has exhibited its maximum dependence at $\omega \simeq 320 \mathrm{MeV}$ and at large backward angles. Among the polarized observables, the beam-target asymmetry $\Sigma_{1 z}$ at $60^{0} \lesssim \theta \lesssim 90^{\circ}$ and the $\Sigma_{2 z}$ at the angle $\theta \lesssim 90^{\circ}$ and around $\omega=450 \mathrm{MeV}$ have shown great response on the change of the mixing ratio. That is, the experiment with linearly or circularly polarized photon off the nucleon polarized in $z$ direction at these angles can bring more infomation on strength of the amplitude $E 2$. 


\section{A. Mandelstam variables}

The kinematics of $s$-channel Compton scattering off the nucleon

$$
\gamma(k)+N(p) \longrightarrow \gamma\left(k^{\prime}\right)+N\left(p^{\prime}\right)
$$

can be described by the three Mandelstam variables $s, t$, and $u$ defined as

$$
s=(k+p)^{2}, \quad t=\left(k-k^{\prime}\right)^{2}, \quad u=\left(k-p^{\prime}\right)^{2},
$$

where $s+t+u=2 m^{2} . m$ is the mass of the nucleon.

$k=(\omega, \boldsymbol{k})$ and $k^{\prime}=\left(\omega^{\prime}, \boldsymbol{k}^{\prime}\right)$ stand for the four momentum of the photon at the intial and the final state respectively. And $p=(E, \boldsymbol{p})$ denotes the nucleon 4-momentum before the scattering, while $p=\left(E^{\prime}, \boldsymbol{p}^{\prime}\right)$ refers to the 4-momentum after the scattering. We can also introduce the new invariants $\nu$ and $\eta$ using variables $s$ and $u$ via

$$
\nu=\frac{s-u}{4 m}, \quad \quad \eta=\frac{1}{m^{2}}\left(m^{2}-s u\right) .
$$

In labortory, center of mass and Breit system these kinematic invariants are expressed as follows:

- In labortory frame $\left(\boldsymbol{p}_{l a b}=0\right)$ the invariants $s, t$ and $u$ read

$$
\begin{aligned}
& s=m^{2}+2 m \omega_{l a b}, \\
& t=-2 \omega_{l a b} \omega_{l a b}^{\prime}\left(1-\cos \theta_{l a b}\right), \\
& u=m^{2}-2 m \omega_{l a b}^{\prime},
\end{aligned}
$$

with

$$
\omega_{l a b}^{\prime}=\omega_{l a b}+\frac{t}{2 m} .
$$

And then

$$
\begin{aligned}
\nu & =\frac{1}{2}\left(\omega_{l a b}+\omega_{l a b}^{\prime}\right), \\
\eta & =2 \omega \omega^{\prime}\left(1+\cos \theta_{l a b}\right) .
\end{aligned}
$$


- In the c.m.s $\left(\boldsymbol{k}_{c m}+\boldsymbol{p}_{c m}=0\right)$ the invariant $s$ corresponds to the square of the total energy of the system $W_{t o t}$, and $t$ is equal to the negative squared of the momentum transfer $\boldsymbol{q}_{c m}=\boldsymbol{p}_{c m}^{\prime}-\boldsymbol{p}_{c m}$ :

$$
\begin{aligned}
& s=\left(\omega_{c m}+E_{c m}\right)^{2}=W_{t o t}^{2}, \\
& t=-2 \omega_{c m}^{2}\left(1-\cos \theta_{c m}\right)=-\left|\boldsymbol{q}_{c m}\right|^{2} .
\end{aligned}
$$

Note that

$$
\omega_{c m}=\frac{\omega_{l a b}}{\sqrt{2 \omega_{l a b}+m^{2}}} .
$$

- In Breit frame $\left(\boldsymbol{p}_{B}+\boldsymbol{p}_{B}^{\prime}=0\right)$ the Mandelstam variables are represented by

$$
\begin{aligned}
& s=m^{2}+2 E_{B} \omega_{B}-\frac{t}{2}, \\
& t=-2 \omega_{B}^{2}\left(1-\cos \theta_{B}\right)=-\left|\boldsymbol{q}_{B}\right|^{2}, \\
& u=m^{2}-2 E_{B} \omega_{B}-\frac{t}{2},
\end{aligned}
$$

where $\boldsymbol{q}_{B}=\boldsymbol{p}_{B}^{\prime}-\boldsymbol{p}_{B}$.

Indeed,

$$
m \nu_{B}=E_{B} \omega_{B}
$$

and

$$
E_{B}=m^{2}-\frac{t}{4}
$$

In particular,

$$
\begin{array}{ll}
\omega_{B}=\omega_{l a b}, & \theta_{B}=0, \\
\omega_{B}=\omega_{c m}, & \theta_{B}=\pi .
\end{array}
$$




\section{B. Symmetry property of $d$-function}

$$
\begin{aligned}
d_{\lambda_{1} \lambda_{2}}^{J}(\theta) & \equiv<J \lambda_{1}\left|e^{-i \theta J_{y}}\right| J \lambda_{2}> \\
& =\sqrt{\frac{\left(J+\lambda_{1}\right) !\left(J-\lambda_{2}\right) !}{\left(J-\lambda_{1}\right) !\left(J+\lambda_{2}\right) !}} \frac{1}{\left(\lambda_{1}-\lambda_{2}\right) !}\left(\cos \frac{\theta}{2}\right)^{\left|\lambda_{1}+\lambda_{2}\right|}\left(\sin \frac{\theta}{2}\right)^{\left|\lambda_{1}-\lambda_{2}\right|} \\
& * F\left(-J+\lambda_{1}, J+\lambda_{1}+1, \lambda_{1}-\lambda_{2}+1, \sin ^{2} \frac{\theta}{2}\right) .
\end{aligned}
$$

Here, $F(a, b, c, \sigma)$ is a hypergeometric polynomial of the $\sigma=\sin ^{2} \frac{\theta}{2}$ :

$$
F(a, b, c, \sigma)=1+\frac{a b}{c} \frac{\sigma}{1 !}+\frac{a(a+1) b(b+1)}{c(c+1)} \frac{\sigma^{2}}{2 !}+\ldots
$$

Space inverse $\left(P: \lambda_{1} \lambda_{2} \rightarrow \lambda_{2} \lambda_{1}\right)$ and time reversal $\left(T: \lambda_{1} \lambda_{2} \rightarrow-\lambda_{1}-\lambda_{2}\right)$ invariance lead to

$$
\begin{aligned}
d_{\lambda_{1} \lambda_{2}}^{J}(\theta) & =(-1)^{\lambda_{1}-\lambda_{2}} d_{\lambda_{2} \lambda_{1}}^{J}(\theta), \\
& =(-1)^{\lambda_{1}-\lambda_{2}} d_{-\lambda_{1}-\lambda_{2}}^{J}(\theta)
\end{aligned}
$$

From Eq. (B.1) it is found that

$$
\begin{aligned}
& d_{3 / 2,3 / 2}^{J}(\theta)=\sqrt{(1-\sigma)^{3}} F(1-L, L+3,1, \sigma), \\
& d_{3 / 2,-3 / 2}^{J}(\theta)=(-1)^{L} \sqrt{\sigma^{3}} F(1-L, L+3,1,1-\sigma), \\
& d_{3 / 2,1 / 2}^{J}(\theta)=-\sqrt{L(L+2)} \sqrt{\sigma(1-\sigma)^{2}} F(1-L, L+3,2, \sigma), \\
& d_{3 / 2,-1 / 2}^{J}(\theta)=-(-1)^{L} \sqrt{L(L+2)} \sqrt{\sigma^{2}(1-\sigma)} F(1-L, 1+3,2,1-\sigma), \\
& d_{1 / 2,1 / 2}^{J}(\theta)=\sqrt{1-\sigma} F(-L, L+2,1, \sigma) \\
& d_{1 / 2,-1 / 2}^{J}(\theta)=-(-1)^{L} \sqrt{\sigma} F(-L, L+2,1,1-\sigma) .
\end{aligned}
$$




\section{Unsubtracted dispersion relation}

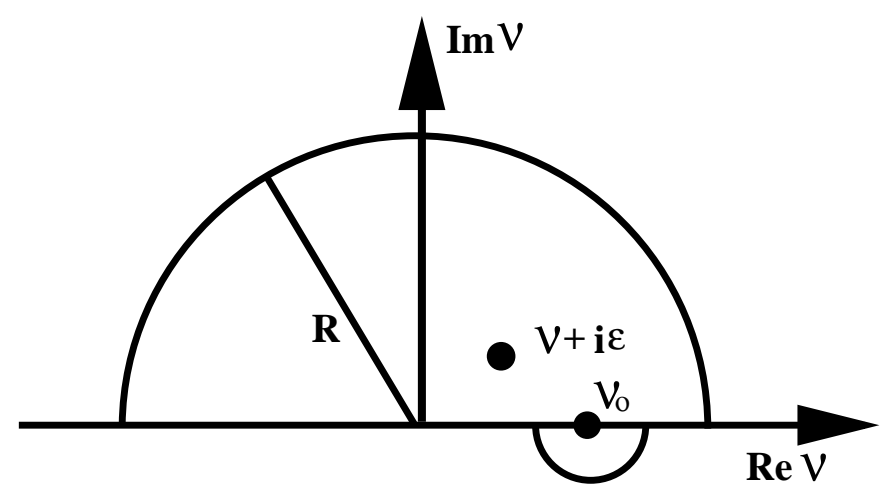

Figure C.1.: The contour of integration $C$ used to derive the dispersion relation.

\section{C.1. Basic assumptions}

Assuming that the scattering amplitude $T(\nu, t)$, that is a function of only $\nu$ for a fixed- $t$, has the following properties [ [ $[2]$ :

1. $T(\nu)$ is analytic for a complex $\nu$ in the region $\operatorname{Im} \nu>0$, but has cuts and poles on the real axis.

2. $|T(\nu)| \leq O\left(|\nu|^{-\alpha}\right)$ as $|z| \rightarrow \infty$ in the upper half of the $\nu$-plane, for some $\alpha>0$. This means that $|T(\nu)|$ decreases to zero asymptotically at least as fast as some negative power of $\nu$.

3. $T\left(\nu^{*}\right)=T^{*}(\nu)$.

\section{C.2. Derivation of basic DR}

On the basis of the analyticity of the scattering amplitude we can evaluate amplitude $T(\nu)$ by using the Cauchy's integral formular, see Fig C.1:

$$
T(\nu+i \epsilon)=\frac{1}{2 \pi i} \oint_{C(R)} \frac{T\left(\nu^{\prime}\right)}{\nu-\nu^{\prime}-i \epsilon} d \nu^{\prime}
$$


By virtue of the second assumption the distant contribution in Eq. (C.1) vanishes at infinity. Eq. (C.1) reduces then to the form

$$
T(\nu+i \epsilon)=\frac{1}{2 \pi i} \int_{-\infty}^{\infty} \frac{T\left(\nu^{\prime}\right)}{\nu-\nu^{\prime}-i \epsilon} d \nu^{\prime}
$$

The integrand has the poles on the real axis as well as the poles due to zero of the denominator. By using the formal identity,

$$
\frac{1}{\nu-\nu^{\prime}-i \epsilon}=\frac{P}{\nu-\nu^{\prime}} \mp i \pi \delta\left(\nu-\nu^{\prime}\right)
$$

together with the third assumption we obtain the usual form of the dispersion relation as follows:

$$
\operatorname{Im} F(\nu)=-\frac{1}{\pi} P \int_{-\infty}^{\infty} \frac{\operatorname{Re} F\left(\nu^{\prime}\right)}{\nu^{\prime}-\nu} d \nu^{\prime}
$$

This connection between a real and an imaginary part of the amplitude is the basic dispersion relation. 


\section{Relations between invariant, helicity and multipole amplitudes}

Here, we present the relations between the three different decompositions $A_{i}, H_{i}$ (or $\left.\tau_{i}\right)$ and $f_{T T^{\prime}}^{L \pm}$ of the scattering amplitude. At first, invariant amplitudes $A_{i}$ are related to reduced helicity amplitudes $\tau_{i}$ as follows [I] :

$$
\begin{aligned}
& A_{1}=\frac{1}{\left(s-m^{2}\right)^{2}}\left\{-\frac{s}{m}\left(1-\sigma \frac{s+m^{2}}{2 s}\right) \tau_{4}-\frac{\sqrt{s}}{2}\left(\tau_{5}+\sigma \tau_{6}\right)\right\} \\
& A_{2}=\frac{1}{\left(s-m^{2}\right)^{3}}\left\{-\frac{s}{m}\left(s+m^{2}\right)\left(1-\sigma \frac{s-m^{2}}{2 s}\right) \tau_{4}-\frac{\sqrt{s}}{2}\left(s-m^{2}\right) \tau_{5}\right. \\
& \left.\quad+2 s \sqrt{s}\left(1-\sigma \frac{s-m^{2}}{4 s}\right) \tau_{6}\right\} \\
& A_{3}=\frac{1}{\left(s-m^{2}\right)^{2}\left(s-m^{2}+t / 2\right)}\left\{m^{3} \tau_{1}+m^{3}(1-\sigma) \tau_{2}\right. \\
& A_{4}=\frac{\left.-2 m^{2} \sqrt{s}\left(1-\sigma \frac{s+m^{2}}{2 s}\right) \tau_{3}\right\}}{\left(s-m^{2}\right)^{2}\left(s-m^{2}+t / 2\right)}\left\{m^{3} \tau_{1}+m^{3}\left(1-\sigma \frac{m^{2}}{s}\right) \tau_{2}-\frac{2 m^{4}}{\sqrt{s}} \sigma \tau_{3}\right\} \\
& A_{5}=\frac{1}{\left(s-m^{2}\right)^{2}\left(s-m^{2}+t / 2\right)}\left\{m\left(s+m^{2}\right) \sigma \tau_{4}-m^{2} \sqrt{s}\left(\tau_{5}+\sigma \tau_{6}\right)\right\} \\
& A_{6}=\frac{1}{\left(s-m^{2}\right)^{2}\left(s-m^{2}+t / 2\right)}\left\{-\frac{m}{2}\left(s+m^{2}\right)\left(\tau_{1}+(1-\sigma) \tau_{2}\right)\right. \\
& \left.\quad+2 m^{2} \sqrt{s}(1-\sigma) \tau_{3}\right\}
\end{aligned}
$$

with

$$
\sigma=\sin ^{2} \frac{\theta}{2}=-\frac{s t}{\left(s-m^{2}\right)^{2}}
$$


The expansion of helicity amplitudes $H_{i}$ by multipoles $f_{T T^{\prime}}^{L \pm}$ are given as [8.5]:

$$
\begin{gathered}
H_{1,5}=\frac{1}{2} \sum_{L=0}^{\infty}(L+1)\left\{(L+2)^{2}\left(f_{E E}^{(L+1)-} \pm f_{M M}^{(L+1)-}\right) \pm L^{2}\left(f_{E E}^{L+} \pm f_{M M}^{L+}\right)\right. \\
\left.\mp 2 L(L+2)\left(f_{E M}^{L+} \pm f_{M E}^{L+}\right)\right\} d_{1 / 2, \pm 1 / 2}^{L+\frac{1}{2}} \\
H_{3,4}=\frac{1}{2} \sum_{L=1}^{\infty}(L+1) \sqrt{L(L+2)}\left\{(L+2)\left(f_{E E}^{(L+1)-} \pm f_{M M}^{(L+1)-}\right) \mp L\left(f_{E E}^{L+} \pm f_{M M}^{L+}\right)\right. \\
\left. \pm 2\left(f_{E M}^{L+} \pm f_{M E}^{L+}\right)\right\} d_{1 / 2, \pm 3 / 2}^{L+\frac{1}{2}} \\
H_{2,6}= \pm \frac{1}{2} \sum_{L=1}^{\infty}(L+1) L(L+2)\left\{\left(f_{E E}^{(L+1)-} \pm f_{M M}^{(L+1)-}\right) \pm\left(f_{E E}^{L+} \pm f_{M M}^{L+}\right)\right. \\
\left. \pm 2\left(f_{E M}^{L+} \pm f_{M E}^{L+}\right)\right\} d_{3 / 2, \pm 3 / 2}^{L+\frac{1}{2}}
\end{gathered}
$$

with $f_{E E}^{0+}=f_{M M}^{0+}=f_{E M}^{0+}=f_{M E}^{0+} \equiv 0$.

Rather accurately, the amplitudes $H_{i}$ and their imaginary parts can be approximated by the pion amplitudes with angular momentum $l \leq 1$. Amplitudes $H_{i}$ are then described by $f_{M M}^{1 \pm}, f_{E E}^{1 \pm} f_{M E}^{1+}$ and $f_{E M}^{1+}$, while $\operatorname{Im} H_{i}$ are mainly obtained from $E_{0+}$, $M_{1-}, E_{1+}$ and $M_{1+}$ by virtue of Eq. (2.26):

$$
\begin{aligned}
H_{1}= & 2 \cos \frac{\theta}{2}\left(f_{E E}^{1-}+f_{M M}^{1-}\right) \\
& +\frac{1}{2} \cos \frac{\theta}{2}(3 \cos \theta-1)\left\{\left(f_{E E}^{1+}+f_{M M}^{1+}\right)-6\left(f_{E M}^{1+}+f_{M E}^{1+}\right)\right\}, \\
H_{2}= & \frac{3}{2} \cos \frac{\theta}{2}(1+\cos \theta)\left\{\left(f_{E E}^{1+}+f_{M M}^{1+}\right)+2\left(f_{E M}^{1+}+f_{M E}^{1+}\right)\right\} \\
H_{3}= & -\frac{3}{2} \sin \frac{\theta}{2}(1+\cos \theta)\left\{\left(f_{E E}^{1+}+f_{M M}^{1+}\right)-2\left(f_{E M}^{1+}+f_{M E}^{1+}\right)\right\}, \\
H_{4}= & \frac{3}{2} \cos \frac{\theta}{2}(1-\cos \theta)\left\{\left(f_{E E}^{1+}-f_{M M}^{1+}\right)-2\left(f_{E M}^{1+}-f_{M E}^{1+}\right)\right\} \\
H_{5}=- & 2 \sin \frac{\theta}{2}\left(f_{E E}^{1-}-f_{M M}^{1-}\right) \\
& \quad+\frac{1}{2} \sin \frac{\theta}{2}(3 \cos \theta+1)\left\{\left(f_{E E}^{1+}-f_{M M}^{1+}\right)-6\left(f_{E M}^{1+}-f_{M E}^{1+}\right)\right\}, \\
H_{6}= & -\frac{3}{2} \sin \frac{\theta}{2}(1-\cos \theta)\left\{\left(f_{E E}^{1+}-f_{M M}^{1+}\right)+2\left(f_{E M}^{1+}-f_{M E}^{1+}\right)\right\} .
\end{aligned}
$$


and

$$
\begin{aligned}
\operatorname{Im} H_{1}= & 2 \cos \frac{\theta}{2}\left(\left|E_{0+}\right|^{2}+\left|M_{1-}\right|^{2}\right) \\
& +\frac{1}{2} \cos \frac{\theta}{2}(3 \cos \theta-1)\left\{9\left|E_{1+}\right|^{2}+\left|M_{1+}\right|^{2}-6 \operatorname{Re}\left(E_{1+} M_{1+}^{*}\right)\right\}, \\
\operatorname{Im} H_{2}= & \frac{3}{2} \cos \frac{\theta}{2}(1+\cos \theta)\left\{\left|E_{1+}\right|^{2}+\left|M_{1+}\right|^{2}+2 \operatorname{Re}\left(E_{1+} M_{1+}^{*}\right)\right\}, \\
\operatorname{Im} H_{3}= & \frac{3}{2} \sin \frac{\theta}{2}(1+\cos \theta)\left\{3\left|E_{1+}\right|^{2}-\left|M_{1+}\right|^{2}+2 \operatorname{Re}\left(E_{1+} M_{1+}^{*}\right)\right\}, \\
\operatorname{Im} H_{4}= & \frac{3}{2} \cos \frac{\theta}{2}(1-\cos \theta)\left\{3\left|E_{1+}\right|^{2}-\left|M_{1+}\right|^{2}-2 \operatorname{Re}\left(E_{1+} M_{1+}^{*}\right)\right\} \\
\operatorname{Im} H_{5}= & -2 \sin \frac{\theta}{2}\left(\left|E_{0+}\right|^{2}-\left|M_{1-}\right|^{2}\right) \\
& -\frac{1}{2} \sin \frac{\theta}{2}(3 \cos \theta+1)\left\{9\left|E_{1+}\right|^{2}+\left|M_{1+}\right|^{2}+6 \operatorname{Re}\left(E_{1+} M_{1+}^{*}\right)\right\}, \\
\operatorname{Im} H_{6}= & \frac{3}{2} \sin \frac{\theta}{2}(1-\cos \theta)\left\{\left|E_{1+}\right|^{2}+\left|M_{1+}\right|^{2}+2 \operatorname{Re}\left(E_{1+} M_{1+}^{*}\right)\right\} .
\end{aligned}
$$




\section{E. Compton scattering amplitudes $R_{i}$ in center of mass system}

In c.m.s the Compton scattering amplitude $T_{f i}$ in Eq. (2.4) can be expressed in terms of six functions $R_{i}(\omega, \theta), i=1 \ldots 6[6.9]$ :

$$
T_{f i}=8 \pi \sqrt{s} \sum_{i=1}^{6} \rho_{i} R_{i}(\omega, \theta),
$$

where $s=(\omega+E)^{2}$. The spin basis $\rho_{i}$ read

$$
\begin{gathered}
\rho_{1}=\boldsymbol{e}^{* \prime} \cdot \boldsymbol{e}, \quad \rho_{2}=\boldsymbol{s}^{* \prime} \cdot \boldsymbol{s}, \quad \rho_{3}=i \boldsymbol{\sigma} \cdot\left(\boldsymbol{e}^{* \prime} \times \boldsymbol{e}\right), \quad \rho_{4}=i \boldsymbol{\sigma} \cdot\left(\boldsymbol{s}^{* \prime} \times \boldsymbol{s}\right), \\
\rho_{5}=i\left(\boldsymbol{\sigma} \cdot \hat{\boldsymbol{k}} \boldsymbol{s}^{* \prime} \cdot \boldsymbol{e}-\boldsymbol{\sigma} \cdot \hat{\boldsymbol{k}}^{\prime} \boldsymbol{e}^{* \prime} \cdot \boldsymbol{s}\right), \quad \rho_{6}=i\left(\boldsymbol{\sigma} \cdot \hat{\boldsymbol{k}}^{\prime} \boldsymbol{s}^{* \prime} \cdot \boldsymbol{e}-\boldsymbol{\sigma} \cdot \hat{\boldsymbol{k}} \boldsymbol{e}^{* \prime} \cdot \boldsymbol{s}\right) .
\end{gathered}
$$

The amplitudes $R_{i}$ are connected to the invariant amplitudes $A_{i}$ by

$$
\begin{aligned}
& R_{1}=C\left\{c_{1}\left(-A_{1}-\frac{W^{2}}{m^{2}} A_{3}\right)-\frac{\nu}{m} c_{2} A_{5}-\frac{W}{m} c_{3} A_{6}\right\}, \\
& R_{2}=C\left\{c_{1}\left(A_{1}-\frac{W^{2}}{m^{2}} A_{3}\right)+\frac{\nu}{m} c_{2} A_{5}-\frac{W}{m} c_{3} A_{6}\right\}, \\
& R_{3}=C\left\{(W-m)^{2}\left((z-1) A_{1}+(z+1) \frac{W^{2}}{m^{2}} A_{3}\right)-\frac{\nu}{m} c_{3} A_{5}-\frac{W}{m} c_{2} A_{6}\right\}, \\
& R_{4}=C\left\{(W-m)^{2}\left((z-1) A_{1}+(z+1) \frac{W^{2}}{m^{2}} A_{3}\right)+\frac{\nu}{m} c_{3} A_{5}-\frac{W}{m} c_{2} A_{6}\right\}, \\
& R_{5}=C\left\{(W-m)^{2}\left(-A_{1}-\frac{W^{2}}{m^{2}} A_{3}\right)-\left(W^{2}-m^{2}\right)\left(A_{2}+\frac{W^{3}}{m^{3}} A_{4}\right)\right. \\
& \left.\quad+2(W-m)\left(-\nu A_{5}+\frac{W^{2}}{m} A_{6}\right)\right\}, \\
& R_{6}=C\left\{(W-m)^{2}\left(A_{1}-\frac{W^{2}}{m^{2}} A_{3}\right)-\left(W^{2}-m^{2}\right)\left(-A_{2}+\frac{W^{3}}{m^{3}} A_{4}\right)\right. \\
& \left.\quad+2(W-m)\left(\nu A_{5}+\frac{W^{2}}{m} A_{6}\right)\right\} .
\end{aligned}
$$


Here, $z=\cos \theta$ and

$$
\begin{gathered}
C=\frac{\left(s-m^{2}\right)^{2}}{64 \pi s^{2}}, \quad c_{1}=4 m W+(W-m)^{2}(1-z), \\
c_{2}=4 W(W-m)-(W-m)^{2}(1-z), \quad c_{3}=4 W^{2}-(W-m)^{2}(1-z) .
\end{gathered}
$$

The invariants $\nu, t$ and $\eta$ are given by

$$
\nu=\frac{s-m^{2}+t / 2}{2 m}, \quad t=\frac{\left(s-m^{2}\right)^{2}}{2 s}(z-1), \quad \eta=\frac{\left(s-m^{2}\right)^{2}}{2 m^{2}}(z+1) .
$$

Due to Eqs. (D.1), (D.1) and (D.3) the amplitudes $R_{i}$ have the following multipole expansions:

$$
\begin{aligned}
& R_{1}=\sum_{L \geq 1}\left[\left\{(L+1) f_{E E}^{L+}+L f_{E E}^{L-}\right\}\left(L P_{L}^{\prime}+P_{L-1}^{\prime \prime}\right)-\left\{(L+1) f_{M M}^{L+}+L f_{M M}^{L-}\right\} P_{L}^{\prime \prime}\right], \\
& R_{2}=\sum_{L \geq 1}\left[\left\{(L+1) f_{M M}^{L+}+L f_{M M}^{L-}\right\}\left(L P_{L}^{\prime}+P_{L-1}^{\prime \prime}\right)-\left\{(L+1) f_{E E}^{L+}+L f_{E E}^{L-}\right\} P_{L}^{\prime \prime}\right], \\
& R_{3}=\sum_{L \geq 1}\left[\left\{f_{E E}^{L+}-f_{E E}^{L-}\right\}\left(L P_{L-1}^{\prime \prime}+L^{2} P_{L}^{\prime}\right)\right. \\
& \left.-\left\{f_{M M}^{L+}-f_{M M}^{L-}\right\} P_{L}^{\prime \prime}+2 f_{E M}^{L+} P_{L+1}^{\prime \prime}-2 f_{M E}^{L+} P_{L}^{\prime \prime}\right], \\
& R_{4}=\sum_{L \geq 1}\left[\left\{f_{M M}^{L+}-f_{M M}^{L-}\right\}\left(L P_{L-1}^{\prime \prime}+L^{2} P_{L}^{\prime}\right)\right. \\
& \left.-\left\{f_{E E}^{L+}-f_{E E}^{L-}\right\} P_{L}^{\prime \prime}+2 f_{M E}^{L+} P_{L+1}^{\prime \prime}-2 f_{E M}^{L+} P_{L}^{\prime \prime}\right], \\
& R_{5}=\sum_{L \geq 1}\left[\left\{f_{E E}^{L+}-f_{E E}^{L-}\right\}\left(L P_{L}^{\prime \prime}+P_{L-1}^{\prime \prime \prime}\right)-\left\{f_{M M}^{L+}-f_{M M}^{L-}\right\} P_{L}^{\prime \prime \prime}\right. \\
& \left.+f_{E M}^{L+}\left\{(3 L+1) P_{L}^{\prime \prime}+2 P_{L-1}^{\prime \prime \prime}\right\}-2 f_{M E}^{L+}\left\{(L+1) P_{L+1}^{\prime \prime}+2 P_{L}^{\prime \prime \prime}\right\}\right], \\
& R_{6}=\sum_{L \geq 1}\left[\left\{f_{M M}^{L+}-f_{M M}^{L-}\right\}\left(L P_{L}^{\prime \prime}+P_{L-1}^{\prime \prime \prime}\right)-\left\{f_{E E}^{L+}-f_{E E}^{L-}\right\} P_{L}^{\prime \prime \prime}\right. \\
& \left.+f_{M E}^{L+}\left\{(3 L+1) P_{L}^{\prime \prime}+2 P_{L-1}^{\prime \prime \prime}\right\}+2 f_{E M}^{L+}\left\{(L+1) P_{L+1}^{\prime \prime}+2 P_{L}^{\prime \prime \prime}\right\}\right],
\end{aligned}
$$

where $P_{L}=P_{L}(z)$ refer to Legendre polynomials of $z$. T-invariance leads to

$$
f_{E M}^{l+}=f_{M E}^{(l+1)-}, \quad f_{M E}^{l+}=f_{E M}^{(l+1)-} .
$$




\section{F. Pauli and Dirac matrices}

\section{F.1. Pauli matrix}

The components of the Pauli spin vector $\boldsymbol{\sigma}=\left(\sigma_{x}, \sigma_{y}, \sigma_{z}\right)$ are

$$
\sigma_{x}=\left(\begin{array}{rr}
0 & 1 \\
1 & 0
\end{array}\right), \quad \sigma_{y}=\left(\begin{array}{rr}
0 & -i \\
i & 0
\end{array}\right), \quad \sigma_{z}=\left(\begin{array}{rr}
1 & 0 \\
0 & -1
\end{array}\right),
$$

and satisfy the relation

$$
\sigma_{i} \sigma_{j}=i \epsilon_{i j k} \sigma_{k}
$$

Here, $\epsilon_{i j k}$ is the antisymmetric tensor:

$$
\epsilon_{i j k}=\epsilon_{j k i}=\epsilon_{k i j}=1, \quad \epsilon_{j i k}=-\epsilon_{i j k} .
$$

The standard commutation and anticommutation relations are

$$
\left[\sigma_{i}, \sigma_{j}\right]=2 i \epsilon_{i j k} \sigma_{k}
$$

and

$$
\left\{\sigma_{i}, \sigma_{j}\right\}=\sigma_{i} \sigma_{j}+\sigma_{j} \sigma_{i}=2 \delta_{i j}
$$

\section{F.1.1. $\quad$ spin matrix}

The spin matrices used in calculation of observables in Eqs. (4.41), (4.43), (4.45), (4.47), (4.49), (4.51) and (4.53) are given by the following tensor product of Pauli matrix:

$$
\begin{gathered}
\sigma_{x} \mathbf{1}=\sigma_{x} \otimes \mathbf{1}=\left(\begin{array}{ll}
0 & \mathbf{1} \\
\mathbf{1} & 0
\end{array}\right)=\left(\begin{array}{llll}
0 & 0 & 1 & 0 \\
0 & 0 & 0 & 1 \\
1 & 0 & 0 & 0 \\
0 & 1 & 0 & 0
\end{array}\right), \\
\mathbf{1} \sigma_{y}=\mathbf{1} \otimes \sigma_{y}=\left(\begin{array}{ll}
\mathbf{1} & 0 \\
0 & 1
\end{array}\right)=i\left(\begin{array}{rrrr}
0 & -1 & 0 & 0 \\
1 & 0 & 0 & 0 \\
0 & 0 & 0 & -1 \\
0 & 0 & 1 & 0
\end{array}\right),
\end{gathered}
$$




$$
\begin{aligned}
& \sigma_{x y}=\sigma_{x} \otimes \sigma_{y}=i\left(\begin{array}{cc}
0 & -\sigma_{x} \\
\sigma_{x} & 0
\end{array}\right)=i\left(\begin{array}{rrrr}
0 & 0 & 0 & -1 \\
0 & 0 & -1 & 0 \\
0 & 1 & 0 & 0 \\
1 & 0 & 0 & 0
\end{array}\right), \\
& \sigma_{z x}=\sigma_{z} \otimes \sigma_{x}=\left(\begin{array}{cc}
\sigma_{x} & 0 \\
0 & -\sigma_{x}
\end{array}\right)=\left(\begin{array}{rrrr}
0 & 1 & 0 & 0 \\
1 & 0 & 0 & 0 \\
0 & 0 & 0 & -1 \\
0 & 0 & -1 & 0
\end{array}\right) \\
& \sigma_{z z}=\sigma_{z} \otimes \sigma_{z}=\left(\begin{array}{cc}
\sigma_{z} & 0 \\
0 & -\sigma_{z}
\end{array}\right)=\left(\begin{array}{rrrr}
1 & 0 & 0 & 0 \\
0 & -1 & 0 & 0 \\
0 & 0 & -1 & 0 \\
0 & 0 & 0 & 1
\end{array}\right) \\
& \sigma_{y x}=\sigma_{y} \otimes \sigma_{x}=i\left(\begin{array}{cc}
0 & -\sigma_{x} \\
\sigma_{x} & 0
\end{array}\right)=i\left(\begin{array}{rrrr}
0 & 0 & 0 & -1 \\
0 & 0 & -1 & 0 \\
0 & 1 & 0 & 0 \\
1 & 0 & 0 & 0
\end{array}\right) \\
& \sigma_{y z}=\sigma_{y} \otimes \sigma_{z}=i\left(\begin{array}{cccc}
0 & -\sigma_{z} \\
\sigma_{z} & 0
\end{array}\right)=i\left(\begin{array}{rrrr}
0 & 0 & -1 & 0 \\
0 & 0 & 0 & 1 \\
1 & 0 & 0 & 0 \\
0 & -1 & 0 & 0
\end{array}\right)
\end{aligned}
$$

\section{F.2. Dirac martrix}

As to the Dirac $\gamma$-matrix

$$
\gamma^{\mu}=\left(\gamma^{0}, \gamma\right)=\left(\gamma^{0}, \gamma^{1}, \gamma^{2}, \gamma^{3}\right)
$$

we use the standard representation in terms of the $2 \times 2$ unit matrix 1 and Pauli matrix $\sigma$ :

$$
\gamma^{0}=\left(\begin{array}{rr}
\mathbf{1} & 0 \\
0 & -\mathbf{1}
\end{array}\right), \quad \gamma=\left(\begin{array}{rr}
0 & \boldsymbol{\sigma} \\
-\boldsymbol{\sigma} & 0
\end{array}\right) .
$$

The important combinations of $\gamma$-matrix components are the traceless product

$$
\gamma_{5}=i \gamma^{0} \gamma^{1} \gamma^{2} \gamma^{3}=\gamma^{5}=\left(\begin{array}{cc}
0 & \mathbf{1} \\
\mathbf{1} & 0
\end{array}\right)
$$

The $\gamma^{\mu}$ matrix obeys the anticommutation relation

$$
\left\{\gamma^{\mu}, \gamma^{\nu}\right\} \equiv \gamma^{\mu} \gamma^{\nu}+\gamma^{\nu} \gamma^{\mu}=2 g^{\mu \nu}
$$


where metric tensor $g^{\mu \nu}=g_{\mu \nu}$ is given as

$$
g_{\mu \nu}=\left(\begin{array}{rrrr}
1 & 0 & 0 & 0 \\
0 & -1 & 0 & 0 \\
0 & 0 & -1 & 0 \\
0 & 0 & 0 & -1
\end{array}\right) .
$$

The matrix $\gamma_{5}$ satisfies

$$
\left\{\gamma_{5}, \gamma^{\mu}\right\}=0 \quad \text { and } \quad \gamma_{5}^{2}=1
$$




\section{Bibliography}

[1] V. A. Petrun'kin A. I. L'vov and M. Schumacher. Phys. Rev., C55(1):359, 1997. 10, 17, 19, 20, [73

[2] S. Hoblit A. M. Sandorfi and J. Tonnison. Few-Body Systems Suppl., 99:1, 1998. [23, 28, 31, 54, 67]

[3] et al. A. Zieger. Phys. Lett., B278:34, 1992. 23

[4] H. D. I. Abarbanel and M. L. Goldberger. Phys. Rev., 165(5):1954, 1968. 18

[5] G. S. Adkins and C. P. P. Nappi. Nucl. Phys., B429:507, 1985. 28, 31

[6] A. J. Buchmann. Phys. Rep., C55:448, 1997. 32

[7] E. Leader C. Bourrely and J. Soffer. Phys. Rep., 59(2):95, 1980. 7

[8] et al. C. Molinari. Phys. Lett., B371:181, 1996. 55

[9] S. Capstick. Phys. Rep., D46:2864, 1992. 23, 31

[10] S. Capstick. Phys. Rep., D46:84, 1992. 23

[11] S. Capstick and G. Karl. Phys. Rep., D41:2767, 1990. 31

[12] S. Capstick and B. D. Keister. Phys. Rev., D51:3598, 1995. 31

[13] K. Ch. Chou and M. I. Shirokov. Sov. Phys. JEPT, 34(5), 1958. 7

[14] H. E. Conzett. Rep. Prog. Phys., 57:1, 1994. 10

[15] A. I. L'vov D. Babusci, G. Giordano, G. Matone, , and A. M. Nathan. Phys. Rev., C58:1013, 1998. [23, 33, [35, 37, 54

[16] G. Giordano D. Babusci and G. Matone. Phys. Rev., C55(4):R1645, 1998. 54

[17] et al. D. Drechsel. Phys. Rev., C61:015204, 2000. 54

[18] G. Krein D. Drechsel and O. Hanstein. Phys. Lett., B420:248, 1998. 27, 29 
[19] R. M. Davidson and N. C. Mukhopadhyay. Phys. Rev. Lett., 79:4509, 1997.55

[20] V. Olmos de León et al. Eur. Phys. J., A10:207, 2001. 28, 31, 67

[21] A. Hügner et al. Nucl. Phys., A620:385, 1997. 54, 55, 56, 58, 61, 63

[22] B. E. Mac Gibbon et al. Phys. Rev., C52:2097, 1995. 19, 23, 54, 56, 58, 61

[23] E. L. Hallin et al. Phys. Rev., C48:1497, 1993. 23, 54, 55, 56, 58, 61, 63

[24] F. J. Federspiel et al. Phys. Rev. Lett., 67:1511, 1991. 23, 54, 56, 58, 61]

[25] F. V. Adamian et al. J. Phys., G19:L139, 1993. 57

[26] G. Bernardini et al. Nucl. Phys., 18:1203, 1960. 54, 58

[27] G. Blanpied et al. Phys. Rev. Lett., 69:1880, 1992. 55

[28] G. Blanpied et al. Phys. Rev. Lett., 76:1023, 1996. 54, 56, 57, 58, 59, 61, 63

[29] G. Galler et al. Phys. Lett., B503:245, 2001. 28, 31, 54, 55, 56, 58, 61, 63, 67

[30] H. Genzel et al. Z. Phys., A279:399, 1976. 54, 56

[31] J. Schmiedmayer et al. Phys. Rev. Lett., 66:1015, 1991. 23

[32] J. W. Dewire et al. Phys. Rev., 124:909, 1961. 54, 56

[33] K. Bermuth et al. Phys. Rev., D37:89, 1998. 32

[34] K. Toshioka et al. Nucl. Phys., B141:57, 1978. 54, 56

[35] K. W. Rose et al. Phys. Lett., B234:460, 1990. 23

[36] M. Bourdeau et al. Phys. Rev. Lett., 58:976, 1987. 31

[37] M. Fiolhais et al. Phys. Lett., B373:229, 1996. 32

[38] M. Jung et al. Z. Phys., C10:197, 1981. 54, 56

[39] M. Warns et al. Z. Phys., C45:627, 1990. 31

[40] P. S. Baranov et al. Sov. Phys. JETP, 23:242, 1966. 54, 55, 58, 61, 63

[41] P. S. Baranov et al. Sov. J. Nucl. Phys., 3:791, 1966. 54, 55, 58, 61, 63

[42] P. S. Baranov et al. Phys. Lett., 52B:122, 1974. 54, 61

[43] R. Beck et al. Phys. Rev. Lett., 78:606, 1997. 55 
[44] T. Ishii et al. Nucl. Phys., B165:189, 1980. 54, 56, 63

[45] W. Broniowski et al. Phys. Lett., B283:22, 1992. 23

[46] Y. Wada et al. Nucl. Phys., 63A:57, 1981. 54, 57

[47] Y. Wada et al. Private Communication., 1984. 54, 56, 63

[48] Yu. A. Kuperin et al. Nucl. Phys., A523:614, 1991. 23

[49] F. E. Low G. F. Chew, M. L. Goldberger and Y. Nambu. Phys. Rev., 106:1345, 1957. ए3.

[50] R. Hagedorn. Relativistic Kinematics. W. A. Benjamin, Inc., 1963. 7

[51] A. C. Hearn. Nuovo Cim., 21:333, 1961. 17

[52] et al. J. Peise. Phys. Lett., B384:37, 1996. 55

[53] S. Hoblit J. Tonnison, A. M. Sandorfi and A. M. Nathan. Phys. Rev. Lett., 80:4382, 1998. [23], 28, 31, 54, 67

[54] J. D. Jackson. Klassische Elektrodynamik. Walter de Gruyter Berlin, New York, 1983. [7, [32

[55] M. Jacob and G. C. Wick. Ann. Phys., 7:104, 1959. 10

[56] G. Kälbermann and J. M. Eisenberg. Phys. Rev., D28:71, 1983. 31

[57] E. Leader. Phys. Rev., 166(5):1599, 1968. 12, 19

[58] K. Y. Lin. Phys. Rev., D24(4):1014, 1981. 23

[59] A. A. Logunov and P. S. Isaev. Nuovo Cim., 10:917, 1958. 17

[60] L.Tiator. Baryons' 98. World Scientific, 1998. 31

[61] M. Lutz and W. Weise. Nucl. Phys., A518:156, 1990. 20

[62] A. I. L'vov. Sov. J. Nucl. Phys., 34:597, 1981. 17, 20

[63] A. I. L'vov and A. M. Nathan. Phys. Rev., C59:1064, 1999. 54

[64] F. E. Low M. Gell-Mann, M. L. Goldberger, E. Marx, and F. Zachariasen. Phys. Rev., 133(1B):B145, 1964. 10, 19

[65] M. L. Goldberger M. Gell-Mann and W. Thirring. Phys. Rev., 95:1612, 1954. 2.5 
[66] S. Mandelstam. Ann. Phys., 21:302, 1963. 9

[67] H. M. Nussenzveig. Causality and Dispersion Relation. Academic Press, Inc., 1972. 8

[68] D. Drechsel O. Hanstein and L. Tiator. Nucl. Phys., A632:561, 1998. 23, 27

[69] V. A. Petrun'kin. Sov. J. Part. Nucl., 12(3):278, 1981. 17, 20, 76

[70] W. Pfeil and D. Schwela. Nucl. Phys., B45:379, 1972. 31

[71] R. E. Prange. Phys. Rev., 110(1):240, 1958. 16

[72] N. M. Queen and G. Violini. Dispersion theory in high-energy physics. Macmillan, London u.a., 1974. 71

[73] I. I. Strakovsky R. A. Arndt and R. L. Workman. Phys. Rev., C53(1):430, 1996. [13, 15, 229, 56

[74] I. I. Strakovsky R. A. Arndt and R. L. Workman. Phys. Rev., C56:577, 1997. [13, 15, 29, 55, 56

[75] S. Ragusa. Phys. Rev., D47:3757, 1993. 23

[76] B. A. Robson. The theory of polarization phenomena. Clarendon Press, Oxford, 1974. उ2

[77] M. De Sanctis and D. Prosperi. Nuovo Cim., A103:1301, 1990. 23

[78] S. Scherer and P. J. Mulders. Nucl. Phys., A549:521, 1992. 23

[79] N. N. Scoccola and W. Weise. Nucl. Phys., A517:495, 1990. 23

[80] B. R. Holstein T. R. Hemmert and J. Kambor. Phys. Rev., D55:5598, 1997. 27, 28

[81] J. Kambor T. R. Hemmert, B. R. Holstein, , and G. Knochlein. Phys. Rev., D57:5746, 1998. 27, 28, 29

[82] J. Kambor V. Bernard, N. Kaiser and U. G. Messner. Nucl. Phys., B388:315, 1993. 27

[83] N. Kaiser V. Bernard and U. G. Messner. Int. J. Mod. Phys., E4:193, 1995. 27

[84] E. M. Lifschitz W. B. Berestetskii and L. P. Pitaevskii. Quantenelektrodynamik. Akademie Verlag GmbH, Berlin, 1991. 7, 12, 33

[85] H. Rollnik W. Pfeil and S. Stankowski. Nucl. Phys., B73:166, 1974. 12, 74 
[86] R. L. Walker. Phys. Rev., 182(5):1792, 1969. 13, 15

[87] L. Li. C. Wang. Phys. Rev., 142(4):1187, 1966. 12

[88] T. Watabe. Phys. Lett., B349:197, 1995. 32

[89] A. Wirzba and W. Weise. Phys. Lett., B188:6, 1987. 31 


\section{Acknowledgement}

In particular, I am grateful to Prof. Dr. M. Schmacher for his suggestion about this thesis, many valuable criticisms, and for the stimulating discussions. I am also indebted to my colleagues for their hospitality and helpful comments.

I would like to express gratitude to Dr. A. I. L'vov for providing his computer code and fruitful discussions.

I would like to thank to my friend, Iris Brunzema, who read my thesis with meticulous care through.

I wish to thank my husband, Hwataek, for his love and encouragement, and my children, who always give me a lot of pleasure in my life.

I acknowledge the financial support of my parents-in-law.

Finally, I would like to show my great thanks to my mother, who has been sacrificing everything to her children. I would like to dedicate this thesis to my father to whom I am greatly indebted for his endless love and security he gave me as he was still alive. 


\section{Lebenslauf}

\author{
Name \\ geboren \\ Familienstand
}

\author{
Ausbildung \\ März 1970-Feb. 1976 \\ März 1976-Feb. 1979 \\ März 1979-Feb. 1982 \\ März 1982
}

Feb. 1986

Sep. 1990

März 1991

Apr. 1991

Apr. 1993-Apr. 1995

27. Apr. 1995

Seit Nov. 1995

Apr. 1997-Juni 1997

Aug. 1997-Juli 1999

Okt. 2000-Juni 2001

\section{Berufliche Erfahrung}

1983-1984

1986-1987

1988-1989
Nan Young Lee

5. 7. 1963 in Seoul, Korea

verheiratet, zwei Kinder

Grundschule "Hong-Ik"

Middle School "Hong-Ik"

High School "Ehwa"

Studienbegin an der Ehwa-Womans-Universität

in Seoul, Korea (Physik)

Studienabschluß mit B.S.

Fortsetzung der Studien in Deutschland

an der Fachbereich Physik

PNdS-Prüfung

Aufname des Physikstudiums

Anfertigung der Diplomarbeit

am Institut für Theoretische Physik

der Universität Göttingen

zum Thema "Selektion quantitativer Merkmale

in Polpulationen mit sexueller Reproduktion"

unter Anleitung von Prof. Dr. R. Kree

Diplomprüfung in Physik

Promotionsstudium am II. Physikalischen Institut

der Universität Göttingen

unter Anleitungung von Prof. Dr. M. Schumacher

Wissenschaftliche Hilfskraft am II. Physikalischen Institut

Stipendium nach Graduiertenförderung

Wissenschaftliche Angestellte am II. Physikalischen Institut

Tätigkeit als Reporterin in der Uni-Zeitung Ehwa

in Korea

Tätigkeit als Reporterin in einem Magazin

Mathematik- und Physiklehrerin

an einer privaten Schule in Korea 